\title{
DEFECTS AND PHASE TRANSFORMATIONS IN \\ COBALT FERRITES
}

Lutgard C. De Jonghe (Yh. L. Thesis)

November 1970

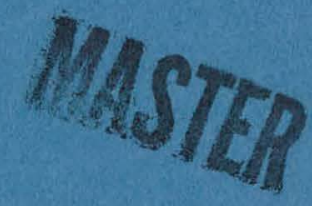

AEC Contract No. W - 7405-eng -48

THIS DOCUMENT CONFIRMED AS UNCLASSIFIED DIVISION OF CLASSIFICATION BY QH Kahn lamb

DATE 21912
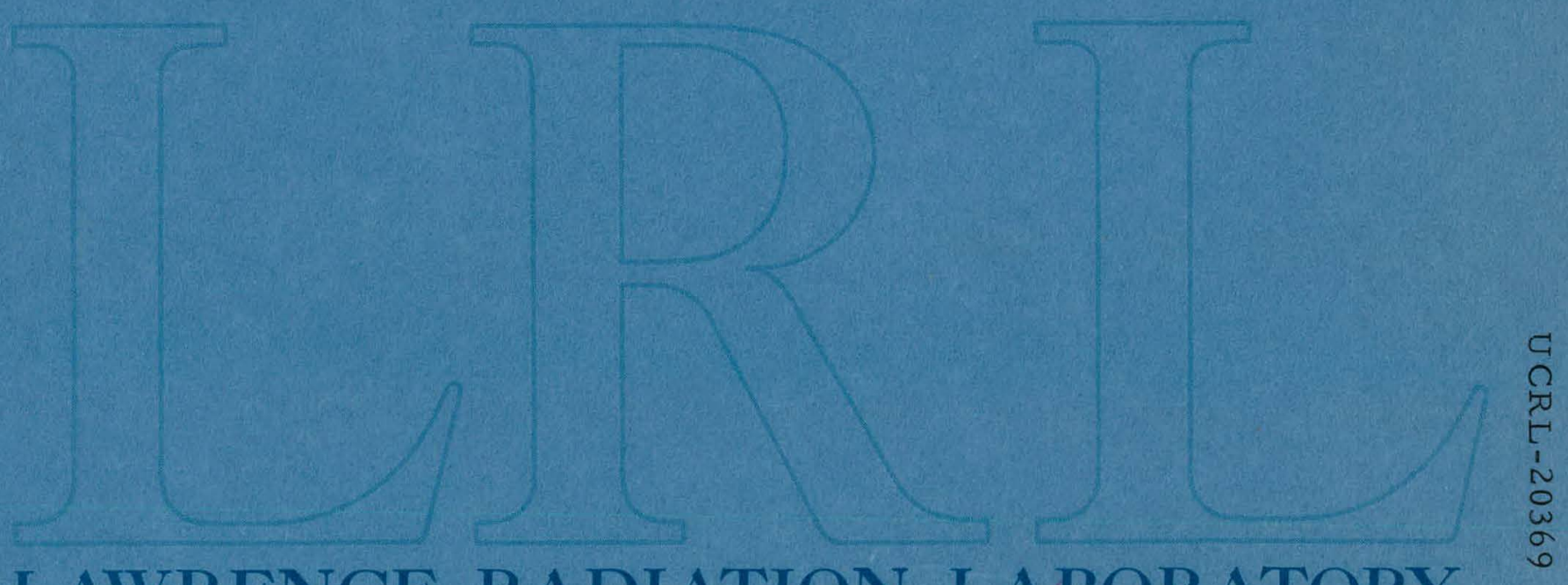

\section{LAWRENCE RADIATION LABORATORY}




\section{DISCLAIMER}

This report was prepared as an account of work sponsored by an agency of the United States Government. Neither the United States Government nor any agency Thereof, nor any of their employees, makes any warranty, express or implied, or assumes any legal liability or responsibility for the accuracy, completeness, or usefulness of any information, apparatus, product, or process disclosed, or represents that its use would not infringe privately owned rights. Reference herein to any specific commercial product, process, or service by trade name, trademark, manufacturer, or otherwise does not necessarily constitute or imply its endorsement, recommendation, or favoring by the United States Government or any agency thereof. The views and opinions of authors expressed herein do not necessarily state or reflect those of the United States Government or any agency thereof. 


\section{DISCLAIMER}

Portions of this document may be illegible in electronic image products. Images are produced from the best available original document. 


\section{PAGES $\mathrm{i}$ to $\mathrm{ii}$ WERE INTENTIONALLY LEFT BLANK}


TAELE OF CONTENT

\begin{abstract}
-
\end{abstract}
I. INTRODUCTION-_- I

II. BACKGROUND-_- 4

A. Crystal Properties-- 4

B. Phase Diagram -

C. Magnetic Properties-_-_- 5

D. Pọint Defect Chemistry-_- 7

III. EXPERTMME'N'IAL-

IV. OBSERVATIONS AND INTERPRETATIONS-_-

$\sqrt{A}$. Radiation Induced Defects-_- 13

B. Dislocations----_- 18

C. Ferrimagnetic Domains-_- 24

D. Phase Transformations-_- 34

E. Transformations in Air- 49

V. FINAL DISCUSSION AND REMARKS-- 62

ACKNOWTEDGEMENTS-_-_-

REFERENCES-D- 67

FIGURE CAPTIONS- 71

FIGURES-C- 77

This report was prepared as an account of work sponsored by the United States Government. Neither the United States nor the United States Atumic Energy Commission, nor any of their employees, nor any of their contractors, subcontractors, or their employees, makes any warranty, express or implied, or assumes any legal liability or responsibility for the accuracy, completeness or usefulness of any information, apparatus, product or process disrinsed, or represents that its use product or process risr.lnsed, or represents
would not infringe privately owned rights. 
DEFECTS AND PHASE TRANSFORMATIONS IN COBAIT FERRITES

Lutgard C: De Jonghe

Inorganic Materials Research Division, Lawrence Radiation Laboratory Department of Materials Science and Engineering, College of Engineering University of California, Berkeley, California

ABSTRACT

Defects and phase transformations in ferrimagnetic cobalt ferrites, with a Co/Fe ratio between 0.54 and 0.76 have been. studied by means of high voltage transmission electron microscopy. The origin and characterization of ion radiation damage, ferrimagnetic domains, dislocations, and phase transformations in air and in the electron microscope are discussed.

Chemical polishing and subsequent irradiation of specimens in the $650 \mathrm{kV}$ microscope produces defects in the crystal surface facing the gun. These defects are mainly spherical oxygen vacancy clusters, but occasionally small prismatic vacancy loops are formed. The projected saturation defect concentration is about $2 \times 10^{9}$ per $\mathrm{cm} .^{2}$

Existing multiple beam dynamical theory has been used to quantitatively compare computed and actual dislocation images. $\therefore$ It has been found that under the proper diffracting conditions, dislocation can be characterized by the spacing of the subsidiary image fringes parallel to the dislocation line, so that the magnitude of the Burgers vector can be determined directly. The dislocation partials are at most $50 \AA$ apart. The usefulness of the transmitted beam image, with systematic higher order reflections operating is shown. This method appears to be preferulle over the kinematical weak bcam method, when the foil is of normal thickness. 
Ferrimagnetic domains have been observed in single phase specimens, when the foils were symmetrically oriented with respect to the field of the objective lens. The domain wall images have been found to be in agreement with the contrast that is expected from $90^{\circ} \mathrm{Bloch}$ walls. The configuration and motion of these $90^{\circ}$ walls has been shown to depend on the foil geometry, and on the presence of mechanical stresses.

Phase transformations in the reducing atmosphere of the microscope have been examined in detail. It has been shown that these transformations are due to the loss of oxygen from the specimen surface. A time dependent gradient in the cation/anion ratio is developed from the surface of the transforming foil, and initially cobalt metal, containing less than 5 at\% iron, has been found to form at the specimen surface. At the same time a very high density of small $(30-40 \AA)$ cobalt-wüstite particles precipitates deeper inside the foil. This (Co,Fe)o has been found to form by a homogeneous transformation. Wedge shaped foils have been observed while transforming, illustrating the dynamical nature of the transformations. After prolonged heating, the foll has been found to consist of cobalt metal grains, and an iron rich spinel matrix. The cobalt grains consist of a mixture of cubic and hexagonal cobalt.

It has been ellown that $(C O, F e)$ precipltates in cobalt ferrite, after heating in air at $1230^{\circ} \mathrm{C}$. The precipitates initially are shaped as square prisms and are fully coherent up to a size of at least $1200 \AA$. Between 1200 and $1500 \AA$ they lose coherency, and also become more or less spherical in shape. The nature of the semi-coherent interface has been discussed in detail. The presence of undissociated interface dislocations has been shown. Micropores were found to migrate along with the semi- 
coherent interfaces. Their origin has been discussed, A possible mechanism by which the precipitates lose coherency has been described. 


\section{INTRODUCTION}

During the last fifteen years transmission electron microscopy has proven its unique usefulness in establishing the relation between the microstructure and the mechanical properties of metals and alloys. It is unfortunate that the electron microscope has not yet established itself in the field of ceramics, where the main tools, beside mechanical testing, remained the optical microscope, or the X-ray diffractometer. More recently the scanning electron microscope has been utilized, but this technique is limited to the characterization of surfaces. One of the reasons that the ceramist usually confines himself to experimental correlations with the macroscopic aspects of the substructure (which in itself has great merit, and is of vital importance to the manufactures of ceramic materials) is that nearly all practical ceramics have an exceedingly complex, multiphase microstructure, from which electron transparent foils are notoriously difficult to prepare. With the commerical introduction of high voltage microscopes, and the development of new foil preparation techniques, such as ion bombardment, most of the experimental difficulties prohibiting a more fundamental characterization of defects and microstructure in ceramic materials, have been overcome. The lack of observations and general information on the sub-micron scale revealed by transmission electron microscopy, makes such a study necessarily of an exploratory nature. To avoid complications, it is also preferable to use single crystals of the ceramic material if they are available.

In the present study defects and phase transformations have been oxamined in single crystals of cobalt ferrites, by means of high voltage 
transmission electron microscopy. This cubic oxide has been selected for a number of reasons: Cobalt ferrite has a spinel structure. Presently, about thirty different cations are known to form spinels with oxygen, 2 and it is hoped that some of the information about cobalt ferrite obtained here, would be relevant to this large class of crystals. Also, cobalt ferrite is ferrimagnetic, and for this reason has found applications in the electronics industries. ${ }^{3}$. Conventionally, grain size or porosity has been used to control the magnetic properties of polycrystalline ferrites, ${ }^{4}$ but it is likely that heat treatments, producing the proper two phase microstructure, could lead to magnetic materials with highly desirable properties. Compared to mechanical properties the field of microstructural relationship with magnetic properties has hardly been explored.

The main emphasis in this thesis is on the phase transformations. This is currently indeed a point of great interest, since, just as for metallic.systems, control of mechanical and electronic properties is possible by the introduction of the appropriate microstructure. At the same time, defects that are well characterized in metals (such as dislocations, or radiation damage produced in the foil during observation in the microscope) need to be examined again in ceramics. While much of the theory of electron image formation can be applied straightforwardly to any crystal, it still has to be made sure that certain contrast effects are not just due to the peculiarities of the crystal structure, rather than to the nature of the defect itself. It seems to the author that meaningful analysis of defects, such as narrowly dissociated dis- 
locations, can only be done if a complete, many beam computer analysis is performed together with the imaging experiments.

Cobalt ferrites are ferrimagnetic, and in a way, magnetic domains can also be classified as defects. A study of domain configurations in the thin films that were examined in the electron microscope, has therefore been included.

The survey nature of the present study made it necessary to describe and analyze many observations. The analyses have given together. with the observations, so as to avoid repetition and impractical cross reference in a separate discussion at the end of all experimental observations. Therefore, each major section has been treated separately, and a summary and conclusions has been given after each one of them. It is hoped that this format improves the clarity of the presentation. A chapter giving some properties, and background about cobalt ferrites has been included. Some general considerations concerining the relevance of this work in the general framework of ceramics have been made in the final discussion. 


\section{BACKGROUND}

\section{A. Crystal Properties}

Cobalt ferrites have a spinel structure. ${ }^{l}$ Spinels are most easily conceived if attention is focused on the oxygen sublattice. The oxygen sublattice is face centered cubic. The octahedral and tetrahedral interstices are partially filled with the cations cobalt and iron. Cobalt ferrites are inverse spinels, which means that the trivalent ions prefer the tetraheral sites rather than the regular octahedral sites. The cation distributions in these spinels have been investigated by many workers. ${ }^{2}$ Still it is not completely clear why one spinel is regular, and another one is inverse. At any rate, the strong preference of cobalt to be in octahedral sites is well established for cobalt fcrritc. Information of the possible ordering of the cations on the octahedral sites is not available, but some octahedral ordering is known for other ferrites. ${ }^{4}$ The difficulty in detecting ordering is that the scattering factors of cobalt and iron are very close. Below is listed the sequence of occupation of the tetrahedral and octahedral sites, when cobalt is progressively substituted for iron in magnetite. The subscript $T$ refers to the tetrahedral intersticial positions, the subscript o refers to the octahedral ones.

$$
\begin{aligned}
& \mathrm{Fe}^{3+}\left(\mathrm{Fe}^{2+} \mathrm{Fe}^{3+}\right) \mathrm{O}_{4} \ldots \ldots \text { magnetite. } \\
& \mathrm{Fe}^{3+} \mathrm{T}^{2+}\left(\mathrm{Co}^{2+} \mathrm{Fe}^{3+}\right) \mathrm{O}_{4} \\
& \mathrm{Fe}^{3+} \mathrm{T}\left(\mathrm{Cu}^{2+} \mathrm{Cu}^{3+}\right) \mathrm{O}_{4} \\
& \mathrm{Co}^{3+}\left(\mathrm{Co}^{2+} \mathrm{Co}^{3+}\right)_{0} \mathrm{O}_{4} \ldots \ldots \text { cobalt spinel }
\end{aligned}
$$


This distribution is shown schematically in Fig. 2, according to Müller et al. 5

The lattice parameters of cubic oxides in the system cobalt-iron, are largely determined by the oxygen ions. The fcc oxygen sublattice parameter for all these phases is around $4.1 \AA$. This was an obstacle to the identification of the phases by electron diffraction. Also, nonstochiometry occurred in the phase transformations studied here, and this again complicated direct diffraction analysis, since it also affects the lattice parameters. In general, however, the nature of the cubic oxides other than the matrix spinel could be deduced from the detailed geometry of the diffraction patterns. What could not be determined was the exact composition of the phases that were present through measurement of the lattice parameters. In certain cases, it has been possible to make estimates of the composition of the reactants less direct.]y.

\section{B. Phase Diagram}

In the literature, two somewhat different phase diagrams are available for one atmosphere of air. Figure la is the one suggested by Aukrust and Muan ; Fig. Ib is the one reported by Robin. ${ }^{6}$ No data are available for other oxygen partial pressures. Although this information would have been valuable here, no attempt was made to make a comprehensive study of the phase diagram of $\mathrm{Co}-\mathrm{Fe}-\mathrm{O}_{2}$ for any range of oxygen partial pressures. This is not the intention of the present work.

\section{Magnetic Properties}

An enormous amount of work has been done on the magnetic properties of spinel ferrites, and most of this has been summarized in a good review paper up to 1968 by Broesse van Groennau et al. 4 
Cobalt ferrites are ferrimagnetic. In ferrimagnetic spinels, the magnetic moments of the ions on the octahedral sublattice are antiparallel to those of the tetrahedral sublattice. In the perfect spinel the octahedral sublattice contains twice as many atoms as the tetrahedral one, so that a spontaneous magnetic moment is present. In Table I the Curie and Nêel temperatures are listed of some cubic oxides, together with their saturation moments, $\mathrm{M}_{\mathbf{s}}$.

TABLE I.

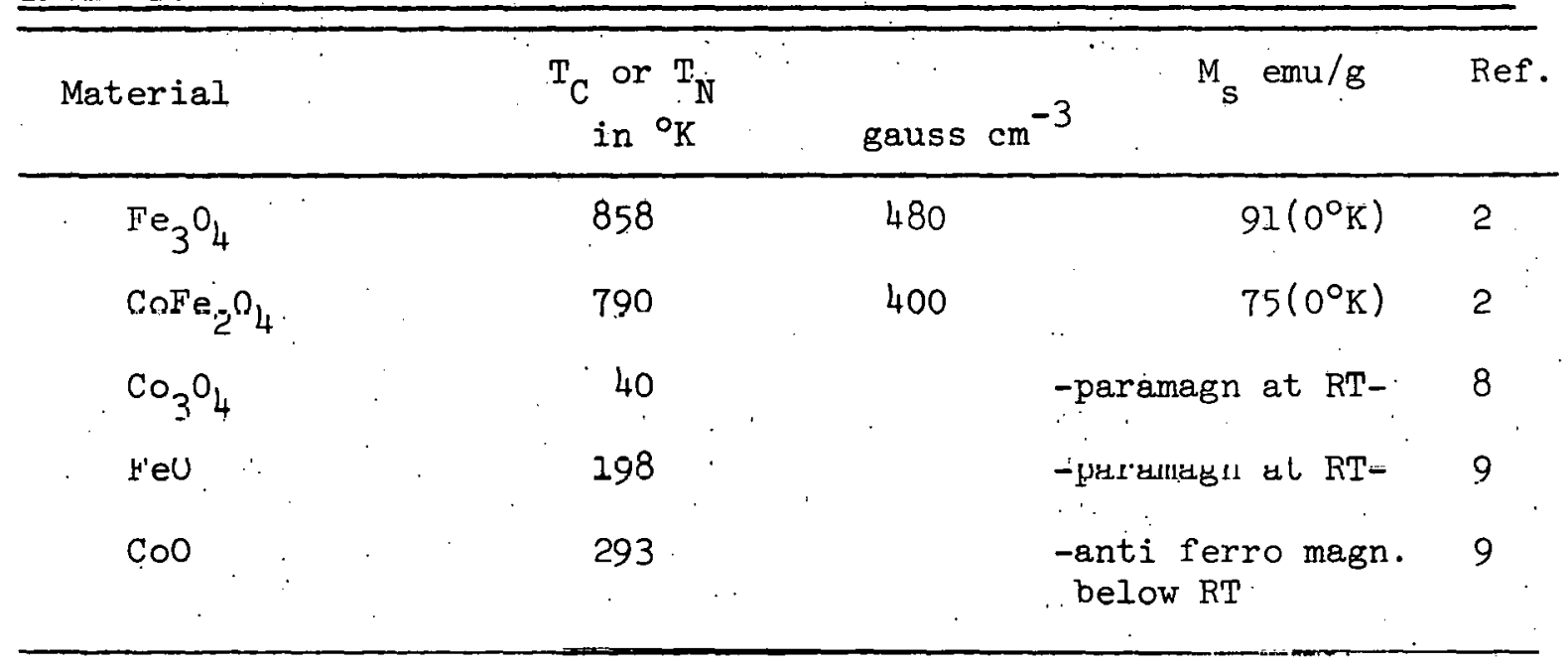

The value of the saturation magnetization of the spinels is temperature dependent below the Curie temperature. $M_{s}$ decreases the closer the measurement is medc to the curle tempcrature. ${ }^{10}$

Substitution of iron by cobalt in $\mathrm{Co}_{1-x} \mathrm{Fe}_{2+x} \mathrm{O}_{4}$ rapidly reduces the Curie temperature when $x<0$. Addition of cobalt to magnetite increases the anisotropy in the favored $(100)$ directions. ${ }^{11}$ Note also that the Néel temperature of cobalteous oxide is about $100^{\circ} \mathrm{K}$ higher than that of wústite. The mixed solid solution oxides ( $\mathrm{Co}, \mathrm{Fe}) \mathrm{O}$ may have a spontaneous magnetic moment 
below their Néel temperature, since the magneton number of an $\mathrm{Fe}^{2+}$ ion is larger than that of a $\mathrm{Co}^{2+}$ ion. The spontaneous magnetic moment of these uncompensated antiferromagnetic oxides should depend strongly on the degree of cation order.

\section{Point Defect Chemistry}

The point defect chemistry of spinels like cobalt ferrite has been studied in detail by Schmaltzreid, Tretjakow, and others. 5,12,13. It appears to be a rather complicated subject. Only the points of importance will be discussed here. Of particular interest is what happens when the partial oxygen pressure in equilibrium with the crystal is reduced. The effect of this can be most easily understood if quasi-chemical reactions of the following kind are considered:

$$
6 \mathrm{Fe}_{0}^{3+}+3 \mathrm{~V}_{0}+\mathrm{Fe}_{3} \mathrm{O}_{4}=9 \mathrm{Fe}_{0}^{2+}+\mathrm{O}_{2}(\mathrm{~g}) \ldots
$$

where $\mathrm{Fe}_{0}^{2+}=$ divalent iron on octahedral interstice

$$
\mathrm{V}_{0}=\text { vacant octahedral site }
$$

The reaction actually states that when the oxygen partial pressure is lowered above magnetite, crystal is destroyed. The produced cations of lower positive valence are placed in vacant: octahedral interstices. For Co $\mathrm{Fe}_{2} \mathrm{O}_{4}$ the reduction reaction will read:

$$
2 \mathrm{Co}_{0}^{3+}+4 \mathrm{Fe}_{0}^{3+}+3 \mathrm{~V}_{0}+\mathrm{CoFe}_{2} \mathrm{O}_{4}=6 \mathrm{Fe}_{0}^{2+}+3 \mathrm{Co}_{0}^{2+}+\mathrm{O}_{2}(\mathrm{~g})
$$

In principle, the concentrations of all the ions and vacancies can be determined. In practice, however, the various equilibrium constants relating the ion and vacancy distributions are nearly impossible to calculate theoretically, and experimentally very dit'ticult to assess, since there are so many variables. Schmaltzreid succeeded in expressing the concentration of the vacancies on the tetrahedral sites, $\left[\mathrm{V}_{\mathrm{T}}\right]$, as a func- 
tion of the trivalent octahedral cobalt and the octahedral vacancies:

$$
\left[\mathrm{V}_{\mathrm{T}}\right]=\mathrm{K}_{1}\left(1-\left[\mathrm{Co}_{0}^{3+}\right]\right)^{-1}\left[\mathrm{~V}_{0}\right]
$$

where $K_{l}$ is a constant depending on the temperature and the Co/Fe ratio. only. As the crystal is reduced, Eq. 2 states that $\left[\mathrm{Co}_{0}^{3+}\right]$ decreases, and according to $\mathrm{Eq} .3$, the ratio of the vacancy concentration $\left[\mathrm{V}_{\mathrm{T}}\right] /\left[\mathrm{V}_{0}\right]$ should decrease. In other words, when the oxygen partial pressure, in equilibrium with a cobalt ferrite spinel, is reduced, the octahedral sites should become more populated than the tetrahedral sites. At the same time Schmaltzreid's calculations show that the cation vacancy concentrations are proportional to the $2 / 3$ power of the oxygen partial pressure that is in equilibrium with the spinel. Schmaltzreid et al. assumed that the oxygen sublattice would essentially be unaffected by the oxygen partial pressure. It should be noted that large oxygen non-stochiometries are in general not supported by these uxide cryslals. If the oxygen pressure is such that large non-stochiometries would result, a phase transformation will take place instead, in which a phase with higher cation/ anion ratio is tormed. As an example:

$$
3 \mathrm{Co} \mathrm{Fe}_{2} \mathrm{O}_{4}=2 \mathrm{Fe}_{3} \mathrm{O}_{4}+3 \mathrm{CoO}+\mathrm{O}_{2}(8) \ldots
$$

A difficulty that is not reflected by the quasi-chemical approach to the point defect situation is that reaction equations such as Eq. 2 and 4 , cannot give information on exactly how the oxygen leaves the crystal when a reduction or a transtormation occurs. At any rate, a gradient of increasing ratio of metal to oxygen should exist from the bulk towards the surface of the oxide that is being reduced. In principle, this gradient can be realized in two different ways: either by a metal ion excess or by an oxygen ion deficiency. In the case of the catio excess, the crystal 
should lose oxygen only at its very surface, and the excess metal ions (of lower valence according to Eq. 2) diffuse inward. If, on the other hand, the reduction creates an oxygen ion deficiency, then oxygen vacancies would be diffusing into the crystal from the surface. The same questions arise when metal is oxidized, and unfortunately no unanimous agreement on the mechanism of oxidation can be found in the literature. For the oxidation of cobalt Valensi, ${ }^{14}$ and Arkharov et al. ${ }^{15}$ support the oxygen vacancy mechanism, while Gulbransen and Andrew ${ }^{16}$ find agreement with Wagner's theory. Bergstein" also speaks of "oxygen diffusing through the crystal" upon reduction or oxidation of copper iron spinels. According to Wagner ${ }^{18}$ it is the cations that are active during oxidation or reduction. While a certain concentration of oxygen vacancies must exist in any oxide crystal, the present author finds the arguments in favor of Wagner's theory more compelling, although the observations in this study can be explained either way. If the oxygen non-stochiometry consists of excess cations, then a study of the oxygen diffusivity as a function of oxygen pressure may confirm this. Indeed, with the cation excess model, the oxygen diffusivity should be relatively independent of oxygen partial pressure, since it is reasonable that oxygen diffuses by a vacancy mechanism over its own sublattice only. The author is not aware of such a study in the literature. As far as phase transformation is concerned, with the cation excess model the rates should be controlled by the mobility of the faster moving cations, or maybe by the rate at which the oxygen desorption at the crystal surface takes place. The diffusion rates of the metal ions will depend on the vacancy concentrations in the cation sublattice, which, as was pointed out earlier, is a function of the oxygen partial pressure. 


\section{EXPERIMENTAI}

Specimens were obtained from the Airtron-Litton Industries, in the form of small single crystals with a diameter of 2 to $3 \mathrm{~mm}$. Analysis of the crystal composition was done by first reducing the crystals to metal by hoating them in lydrogen. Then lle Cu/Fe rullu was determined with. the standard chemical techniques. The composition of the crystals varied between $\mathrm{Co}_{1.05} \mathrm{Fe}_{1.95} \mathrm{O}_{4}$ and $\mathrm{Co}_{1.29} \mathrm{Fe}_{1.71} \mathrm{O}_{4} \cdot$ The as received crystals were annealed for about fifty hours in the solid solution range at $900^{\circ} \mathrm{C}$. Then they were air cooled. The specimens were ground down to platelets with a thickness of about 75 micron, and subsequently polished. A chemical polishing technique was used similar to the one reported by Lewis ${ }^{19}$ for magnesium aluminate spinels. 'l'he platelets were immersed in hot phosphoric acid kept at a temperature of about $400^{\circ} \mathrm{C}$. When the platelets had dissolved to about $1 / 3$ of their original size, electron transparent regions could usually be found at their edges. The chemical polishing took five to ten minutes. Immediately after the polishing, the specimens were washed in boiling water, and then in alcohol. The foils were examined in a $650 \mathrm{kV}$ Hitachi transmission electron microscope.

Some phase transformation were followed in situ, with the use of a. hot stage specimen holder. The temperature was controlled indirectly by fixing the power input to the furnace type heater of the hot stage specimen holder. Calibration of the hot stage was therefore necessary. The calibration was carried out in a vacuum bell jar, and the temperature of a specimen was measured with a thermocouple, as a function of power input and time. The calibration curve, Fig. 3a, shows that the temperature of 
the specimen rises to near equilibrium in about $15 \mathrm{~min}$. The curves were determined by turning the power input suddenly to a desired value, and by adjusting the controls such that the power input remained constant in time. Figure $3 b$ shows the equilibrium temperature of the hot stage versus the power input. The maximum cooling rate from $740^{\circ} \mathrm{C}$ is shown in Fig. 3c. Since the atmosphere composition was of fundamental importance in hot stage transformations, it. was analyzed at the specimen stage level with a Varian partial pressure gauge. The total pressure for gases with a mass between $I$ and 70 was measured to be about $5 \times 10^{-6}$ Torr. Figure 4 shows the readout of the gage, from which the partial pressures could be calculated. Water vapor was the main gas phase, which is common in unbaked vacuum systems. The partial pressures of the most important components in the specimen neighborhood have been listed in Fig. 4. Note that the atmosphere was not in equilibrium, since it contained more free hydrogen than free oxygen. The presence of this free hydrogen (and also the helium) may be explained by the dependence of the pumping rate of the diffusion pumps, on the molecular weight of the gas that is being pumped. Low molecular weight gases are pumped less efficiently. It was expected that, when in situ transformations were carried out, this atmosphere would react to equilibrium at the hot specimen surface. The result was then that the excess of hydrogen together with the water vapor, determined the actual oxygen partial pressure. For a given temperature, the oxidizing power of the atmosphere could be found from the ratio $\mathrm{H}_{2} / \mathrm{H}_{2} \mathrm{O}$. For most of the hot stage experiments, this ratio was between 0.1 and 0.05 . Such an atmosphere would e.g. at $500^{\circ} \mathrm{C}$ reduce $c 00$ to metallic cobalt, but it would not reduce Feo. 
No special attempt was made to control the atmosphere of the electron microscope. The composition of this atmosphere did indeed not remain constant over extended periods (days), so in order to have comparable transformation data, the hot stage experiments were carried out when the prevailing atmosphere were comparable. 


\section{OBSERVATIONS AND INTERPRETATIONS}

\section{A. Radiation Induced Defects}

It is well known that the observation of materials in a transmission electron microscope can lead to artifical introduction of defects. These defects, that are not characteristic of the original bulk crystal, are produced by the electron beam or by negative ions that are accelerated together with the electrons. This radiation damage can be very serious if the electrons are accelerated above a certain typical threshold value. As an example, stacking fualts images in $\mathrm{Al}-1 \% \mathrm{Ag}$ alloys are rapidly perturbed by primary electron knock-on damage, when the electron beam is accelerated above $500 \mathrm{kV} .^{20}$ The radiation damage, whether produced by electrons or ions, usually manifests itself in the specimen as very small defect clusters. It might be difficult to distinguish these defects clusters from small precipitates that result from phase transformations. The behavior of single phase crystals of cobalt ferrite during the observation in the high voltage microscope, must be examined for this reason. Numerous small defects were found in all the ferrites that. were observed in the $650 \mathrm{kV}$ electron microscope for any length of time. Figure 5 shows the distribution of the defects around a nearly perfect conical etch pit. The observations were made at an accelerating voltage of $650 \mathrm{kV}$, and this allows the foils to be electron transparent with good resolution up to about 1 micron, for $\bar{g}=440$. When the number of defects per unit area was counted in this foil it was found that the projected defect density was invariant over the entire range of thicknesses. The defect images have black and white lobes, with a Iine of no contrast dividing the lobes. 
These kinds of images typically result from the matrix strain field around defects that are too small to be resolved directly. The contrast of such defects was first discussed by Ashby and Brown; ${ }^{21}$ and subsequently examined in more detail; particularly with regard to depth dependence, by several authors, e.g. Rühle et $a . .^{22}$ The direction of the white-to-black contrast may be characterized by a vector $\bar{q}$ (see Bell and Thomas). ${ }^{23}$ Consideration of the direction of the line of no contrast LC, $\bar{q}$, and $\bar{g}$, can for certain foil orientations allow the immediate identification of the displacement vector associated with the defect. LC and $\bar{q}$ have been defined in the insert in Fig. 5. In this bright field image $\bar{q}$ is the same for every defect, suggesting that they are all located near the top or near the bottom surface of the foil. In the many foils that were examined these def'ects were found with uniform projected density right up to the foil edges. At $650 \mathrm{kV}$ the extinction distance for 440 reflections was calculated to be about $800 \mathrm{~A}$ for cobalt ferrites, so that it may be assumed that the defects are located within half an extinction distance from either surface. Further information must come from a comparison of bright and dark field images. 23 Image contrast calculations according to the procedures set up by Bell and Thomas ${ }^{24}$ for a systematic twelve beam dynamical case at $650 \mathrm{kV}$, showed that, as far as strain contrast from small defects is concerned, the same rules applied as were worked out by Ashby the Brown for a two beam case at $100 \mathrm{kV}$, when the defects are within half an extinction distance from the surface. Bright and dark field images are compared in Figs. $6 a$ and $6 b$. Note that $\bar{q}$ is in the same direction in both bright and dark field. From these observations it could be concluded that the defects were all near the surface of the foil that 
is facing the electron gun, and that they were of the vacancy type. For most of the defects LC is perpendicular to the operating diffraction vector $\bar{g}$, while the direction of $\bar{q}$ is antiparallel to $\bar{g}$. Some defects, for which LC is not perpendicular to $\bar{g}$, have been marked L: For these defects $I C$ is along the [IĪO] direction while $\bar{q}$ is still antiparallel to $g=404$. This strongly suggests that the marked defects are vacancy loops on the (III) plane. The magnitude of the Burgers vectors has not been determined, but the loops are probably perfect prismatic loops with $b=a / 2(110)$. Figures $7 a$ and $7 b$ show two dark field.images with different 440 operating vectors. A loop found in this area is marked L. For the majority of the defect images LC is at all times perpendicular to $\bar{g}$ while at the same time $\bar{q}$ is antiparallel to $\bar{g}$. It must be concluded that the large majority of the defects are spherical vacancy clusters, while only occasionally a prismatic loop is formed.

The increase in projected defect density with constant irradiation in the microscope has been followed. Figures $8 \mathrm{a}$ to $8 \mathrm{~d}$ show how the defect density changes with time. The diffracting vectors are different 440 reflections. This again allows distinction between loops and spherical clusters. A loop that was found in this area has been marked L. Note that the defects retained their character within the observations interval: there was no switch from spherical cluster to loop, or conversely. The projected defect density versus time is plotted in Fig. 9. These observations showed that the projected defect density saturated at about $2 \times 10^{9}$ per $\mathrm{cm}^{2}$, after about 20 minutes of observation. This kind of behavior was quite similar to that observed by Makin on copper. 20 In the present case the clusters did not continue to grow, however, beyond a radius of 
about $100 \AA$, at least within the period of observation. In appearance, the defects were similar to those resulting from the ion bombardment of some fec metals. ${ }^{25}$ The oxygen vacancies needed for the formation of the defects were probably introduced during the foil preparation. Indeed, during polishing in hot phosphoric acid, hydrogen is produced on the surface of the dissolving crystal. The hydrogen reduces the crystal over a very small depth, which catalyzes the dissolution. The precipitation of the vacancies would then be stimilated by the radiation damage produced during observation in the microscope. The radiation damage resulted from the impact of accelerated ions; it was unlikely that it would originate in primary electron knock-on events, since then the defects should appear simultaneously in the top and the bottom surface of the foil. Also, the def'ects f'ormed with equal density in parts of the foil that were not electron irradiated. The accelerated ions were probably former from the gases that were present in the microscope atmosphere, and were most probably oxygen ions from dissociating water vapor, and nitrogen ions.

Upon heating in the hot stage the defects slowly disappeared at about $400^{\circ} \mathrm{C}$. Above these temperatures phase transformations occurred in the foil and the behavior of the clusters could no Ionger be followed. Defect clusters were also observed to form during observation at. liquid nitrogen temperatures. These defects are shown in Fig. 10. Since there were no tilting facilities available for the cold stage, the number of diffraction experiments that could be carried out were very limited. Still, it seems that the strain fields of the defects are not 
quite spherical. Instead, the strain contrast indicates that at this temperature small stacking fault tetrahedra form (strain contrast from small tetrahedra has been discussed by Chik. ${ }^{26}$ ) THis change of defect character with temperature is not clearly understood, unless one would assume that the stacking fault energy decreases with decreasing temperature.

In summary:

(a) Damage produced by the impingement of accelerated ions stimulates the precipitation of vacancy clusters in the surface of the specimen that is facing the electron gun of the transmission electron microscope. The oxygen vacancies that are needed for the formation of these clusters are probably produced by surface reduction during the polishing in hot phosphoric acid.

(b) The ions that stimulate the defect formation are most likely to be nitrogen ions, and oxygen ions from the dissociation of the gases that are present in the microscope atmosphere.

(c) Near room temperature the defects are nearly all spherical vacancy clusters, while occasionally a perfect prismatic vacancy loop is formed. :There is no switch from spherical clusters to loops, or conversely. At low temperatures, the indications are that small stacking fault tetrahedra are more frequent. The defects can be electrically neutral by a reduction in valence of the neighboring cations.

(d) As with certain metals, care must be exercised in electron microscope analyses of lattice defects in ceramic materials. In the present case, the radiation induced defects must be distinguished from characteristic defects and small precipitates. The maximum projected density of the noncharacteristic defects is about $2 \times 10^{9}$ per $\mathrm{cm}^{2}$, which 
clearly allows the study. of other defects of interest in the foils without interference.

\section{B. Dislocations}

Dislocations in oxide crystals have been somewhat neglected by the ceramist, mainly because most highly ionic crystals are very brittlc, even up to high temperatures. The efforts in studies of mechanical properties of ceramics have therefore been concentrated on crack formation and propagation on a macroscopic scale. Dislocations plays a fundamental role in the mechanical behavior of oxide materials, as they do in metals, and the details of the dislocation structure itself should be of great importance with regard to high temperature deformation, fracture, or creep. Detailed contrast experiments have therefore been carried out to determine the degree of dissociation of dislocations in cobalt ferrite spincls. The geometry of dislocation dissoriation in the spinel lattifse has been discussed in detail by Hornstra. 27 Following considerations made for corundum by Kronberg, ${ }^{28}$ Hornstra advanced arguments for a four-fold dissociation of a perfect dislocation in spinels. The main agruments are that local electroneutrality can only be maintained in the four-fold dissociated configuration and that in nature spine]s are found that are twinned on \{1li\} planes. This observation of twinning in natural spinels was seen as evidence for the presence of Shockley partials. The dislocation reactions are shown in Fig. 1l. For two-fold dissociation the bounding partials have identical Burgers vectors both equal to a $/ 4(110)$. They are bounding a stacking fault in the cation sublattice only, since the fcc oxygen sublattice has actually half the lattice parameter of the spinel 
lattice. Two-fold dissociation was observed in magnesium aluminate spinels, and the nature of the cation stacking fault has been discussed by Lewis. 29 For the four-fold dissociation the two outer pairs of Schockley partials bound a compound fault: an intrinsic stacking fault in the oxygen sublattice plus a fault in the cation sublattice. In this fault, the cations would have moved to their favored crystal coordination. This process was termed synchroshear by $K r o n b e r g$, since it requires the simultaneous diffusional displacement of cations when the dislocation is moved through the crystal. Such a dislocation would be self pinning.

The cobalt iron spinels examined here were grown by a flux method, and contained only few dislocations. Figure 12 shows a network of dislocations that was found in this material. The occurence of networks of this kind immediately rule out that the network dislocations would be Shockley partials, since the oxygen stacking faults cannot be accomodated. Other isolated dislocations that were found, Fig. 13, appeared nat to be resolvably two-fold dissociated either, contrary to what might be expected from the considerations made by Hornstra. No diffracting conditions could be found where fault contrast between dislocation pairs was in evidence. This in itself is already a strong indication that the isoLated disloculions do not have a Burgers vector of a/4 110$\rangle$, but a more positfve conclusion could be drawn if the magnitude of the Burgers vector could be determined more directly. Generally a method is used for this where the dislocation crosses an extinction contour. 30 In strictly two bcam diffracting conditions, as can be more closely approximated in $100 \mathrm{kV}$ electron microscopes, the distinction between $\vec{g}: \vec{b}=1$ and $\vec{g} \cdot \vec{b}=$ ? is 
relatively clear: when the deviation parameter $w_{g}=0$. For $\vec{g} \cdot \vec{b}=1$ the dislocation images exhibit one fringe, while for $\vec{g} \cdot \vec{b}=2$ there are two fringes. At $650 \mathrm{kV}$ the Ewald sphere has appreciably. less curvature than at $100 \mathrm{kV}$, and it is usually not possible to produce two beam diffracting conditions. Instead the specimen should be tilted such that a single crystal zone is reflecting. The image analysis with such a set of systematic reflections requires the application of a many beam dynamical theory. An incremental solution of the many beam dynamical equations has been worked out by Bell and Thomas 24 , and was used in the present analysis.

In Fig: 14 a microphotometer tracing of the dislocation shown in the insert, is compared with calculated profiles. In principle a direct comparison between a microphotometer tracing of an inage and its calculated (ör actual) intensity profile, is not a correct procedure, since for high energy electrons the emulsion density on the photographic plate is linearly related to the incident electron intensity ${ }^{27}$ (and not logarthimically as is the case for photons). 32 Instead, an intensity profile should be compared with a densitometer tracing. There are; however, numerous factors that can affect the actual image profiles, diffuse scattering in particular. The effect of the diffuse scattering, which has not been incorporated in the computer simulations, is to proportinately increase the low intensity levels much more than the near background ones. As $A$. result, the relative differences of the intensity extremes of the actual image profiles will be much less pronounced than the theoretical ones. The exponential response of the microphotometer to emulsion densities ${ }^{31}$, 
exaggerates the intensity minima, and suppresses the maxima. The result. is that the microphotometer partially compensates for the effect of diffuse scattering, so that this tracing actually appears to be closer to the calculated image profiles than a densitometer tracing. The position, however, of the relative minima and maxima should not be affected by the diffuse scattering. It is then better to think of the dislocation image as a set of fringes parallel to the dislocation core, for which the fringe spacings is more characteristic than the fringe intensities. The criterion of agreement of fringe spacing between calculated and actual images will then be the basis for determining the value of $\vec{g} \cdot \vec{b}$. The operating vectors in a set of systematic reflections are labeled $g_{1}, g_{2}$, etc. and their corresponding dimensionless deviation parameters are $\mathrm{w}_{1}$, $\mathrm{w}_{2}$ etc., as is schematically in Fig. 15. The profiles for various values of $\bar{g}_{1} \cdot \bar{b}$, and for various values of $w_{1}$ are then calculatcd. Other parameters are foil thickness, absorption coefficients, and position of the dislocation. In the analyzed dislocation images the foil thickness was determined by counting the number of extinction fringes when $\mathrm{w}_{440}=0$. The extinction distance for a 440 reflection is about $800 \AA$ The first. order extinction distances are rather large; $2600 \AA$ for a 400 reflection, and $2150 \AA$ for a 440 reflection. The scattering potentials were calculated from the scattering factors of the perfect spinel lattice. The program for calculating scattering factors is available."

\footnotetext{
*Glen Stone, Department of Materials Science and Engineering University of California, Berkeley
} 
In Fig. 14, the direction of the Burgers vector was determined by the usual diffraction experiments. With the diffraction geometry of Fig. 19, only $\bar{g}_{1} \cdot \bar{b}=1$ and $\bar{g}_{1} \cdot \bar{b}=2$ are possible. From the fringe spacings of the image, it can be concluded immediately that the value of $\overrightarrow{\mathrm{g}}_{1} \cdot \overrightarrow{\mathrm{b}}=2$. $\Lambda$ comparison between a calculated and an actual dislocation image forced with $\vec{g}_{1} \cdot \vec{b}=1$, is shown in Fig. 16; in this image the geometry is such that $\vec{g}_{1} \cdot \vec{b}=1$. for the undissociated dislocation. Note that the microphotometer tracing agrees in appearance quite well with the calculated intensity profile. The reason for this phenomenon was discussed earlier. Figure 16 shows an unexpected double peak in the image near the core of the dislocation. The doubling of the central fringe is only observed when the image is in focus, so that electrical charges on the dislocation, or possible magnetic inhomogeneities near the core carnot be responsibile. So there is no way in which a splitting of the centra. fringe can be accounted for in an undissociated dislocation, even if this image is from a pure edge dislocation, and the calculated profiles are for pure screws. ${ }^{33}$ Instead, it must be concluded that this dislocation is probably slightly dissociated, with a partial separation of about 50 to $100 \AA$. It is possible that the extent of the dissociation depends on the temperature at which the dislocation was introduced. It is interesting to compare the results of the direct beam imaging with higher order reflections operating, to the high resolution technique that images $\overrightarrow{-g}$ when $+\vec{g}$ is on the reflecting sphere. ${ }^{34}$ A high resolution weak beam experiment is shown in Fig. 17. As is immeidately clear, the high resolution is only obtained in portions where the foil is very thin, and 
deteriorates rapidly with increasing thickness. At the node in.Fig. 17, the foil is about $2000 \AA$ thick. Also, the effective extinction distance in the weak beam mode is of the order of $100 \AA$, which complicates the image analysis tremendously. Nevertheless, the weak beam method is extremely useful when the experimental conditions are favorable. In the more general case, the high order $\vec{g}$ bright field images seem more informative, and deserve more attention than they are currently given in the literature.

All the above information confirms that the cation stacking fault energy is high, and that the dislocations are at most separated by about $50 \AA$. For accurate measurements of dislocation spacings of this order, an in depth study of the contrast from closely spaced dislocations is necessary.

The ferrites that were examined here had a composition that ranged from $\mathrm{Co} / \mathrm{Fe}=0.54$ to 0.76 . The present observations do not of course rule out that for spinels outside this composition interval, the dislocations are indeed more widely separated. It is believed, however, that it can be taken as a general rule that the oxygen stacking fault energies are very high in strongly ionic crystals. This generalization is supported by observations on a number of different oxides. $35-40$

In summary:

(a) The usefulness of the transmitted beam images with higher order reflections on the Ewald sphere was shown. This method seems more generally applicable than the weak beam imaging techniquc which applies only to thin foils.

(b) The actual and the computed images were quantitatively compared. $\Lambda$ systematic twelve beam dynamical program was used in the analysis. 
(c) For the same $w_{1}$, the fringe spacings of the dislocation images are characteristic for the value of $\bar{g}_{1} \cdot \bar{b}$, where $\bar{g}_{1}$ is the lowest order reflection (not necessarily the strongest) of a systematic set.

(d) The dislocations in cobalt ferrite are not dissociated more than $50 \AA$, indicating that the cation stacking fault energy is high. No stacking faults in the oxygen sublattice were observed.

\section{Ferrimagnetio Domains}

In the absence of strong applied fields, all large magnetic crystals contain magnetic domains: In metals, such as cobalt and iron, these domains have usually been imaged in the electron microscope with the Lorenz method. 30 This is essentially an out of focus technique, in which use is made of the differently. directed Lorenz forces in adjacent domains on the probing electron beam. The specimen is usually lifted out of the objective lens, so that it interacts less with the magnetic field of this lens. This method has the unavoidable drawback that crystal defects and domain walls cannot be observed simultaneously. In the present study the ferrimagnetic cobalt ferrite foils were in the regular specimen position, and consequently, as will be discussed below, only $90^{\circ}$ domain walls could be present. $90^{\circ}$ walls can be imaged in focus, so that it would be possible to observe interactions between defects and domain walls more clearly. These interactions were not studied this time, but the observed ferrimagnetic domains, and their behavior in the thin films of cobalt ferrite will be described in some detail. To the author's knowledse, electron microscope observations of ferrimagnetic domain walls in cobalt ferrites have not been reported in the literature yet, and 
even good in focus images of $90^{\circ}$ walls in other materials are very few.

1. Relative Importance of the Magnetization and the Magnetostriction on the Electron Image Formation

The maximum change in the deviation parameters $\bar{s}$, caused by the saturation magnetization $\bar{M}$ inside the ferrimagnetic domains, can be estimated for a certain foil thickness. For the geometry where the magnetization vectors are in the plane of the foil, and anti-parallel across a domain wall, the difference in direction of the probing electron wavefronts at the buttom surface of the foil will be maximum. In all other cases, the effect of the $\overline{\mathrm{M}}$ on the electron image is less. The angular deflection of the electron beam ${ }^{30}$ is seen in Fig. 18.

$$
\phi=4 \pi \text { e } \lambda \mathrm{t} \mathrm{M} / \mathrm{ch}
$$

where

$$
\begin{aligned}
\mathrm{e} & =\text { electron charge in esu } \\
\mathrm{c} & =\text { velocity of light in } \mathrm{cm} \mathrm{sec}^{-1} \\
\mathrm{~h} & =\text { Planck's constant in erg } \mathrm{sec} \\
\lambda & =\text { eleclrul wavelength, } 0.0118 \AA \text { at } 650 \mathrm{kV} . \\
\mathrm{t} & =\text { foil thickness in } \mathrm{cm} \\
\mathrm{M} & =\text { saturation magn. }: 400 \text { Gauss } / \mathrm{cm}^{3}
\end{aligned}
$$

For a foil of $2000 \mathrm{~A}$ thick, observed at $650 \mathrm{kV}, \phi$ is found to be equal to $3.1 \times 10^{-5}$ radians. So even in the most farorable case only a slight elongation of the diffraction spots is to be expected when a diffraction pattern is taken from a region containing a ferrimagnetic domain wall. If observed in the condition where $g=440$ (extinction distance $\xi_{440}=800 \AA$ ), the change in the dimensionless deviation $w$, for a foil of about $2000 \AA$ thisk is: 


$$
\Delta \mathrm{w}=g(2 \phi) \quad \xi_{440}=0.035
$$

Generally, a lattice contraction accompanies magnetic ordering. This effect is called magnetostriction. While in metals the effects of magnetostriction on the electron images usually. can be neglected, this appears not to be like case in cobalt ferrites. The magnetostriction constant for ordering of the spins in $(100)$ directions, $\lambda_{100}$, determined by Bozorth et al ${ }^{41}$ for a ferrite of composition $\mathrm{CO}_{0.8} 8^{\mathrm{Fe}} 2.2^{\mathrm{O}_{4}}$ is:

$$
\lambda_{100}=5.9 \times 10^{-4}
$$

$\lambda_{100}$ expresses the fractional change in lattice parameter. This lattice contraction makes the spinel unit cell slightly tetragonal, giving rise to a coherent domain wall boundary at the magnetic domain wall. For a $180^{\circ}$ Bloch will there is nu difference in luttice oriantation upon crossing the wall, and magnetostriction does not contribute to the image of that wall. Of importance then is contrast from a $90^{\circ}$ wall. 'Ihe geometry of such a wall is analyzed in Fig. 19. Comparable considerations were made for antiferromagnetic walls by Remaut et al. ${ }^{42}$ To make a coherent twin boundary on the diagonal plane, the lattices have to be rotated with respect to each other. The lattice rotation across the domain wall is :

$$
\alpha=2 \operatorname{arctg}\left(\lambda_{100} / \sqrt{ } 2\right) \text {, which since } \alpha \text { is small, can be approximated }
$$
well by:

$$
\alpha=\sqrt{ } 2 \lambda_{100}=8 \times 10^{-4} \mathrm{rad}
$$

If the axis of rotation is contained in the foil plane, this will give rise to a change in $w$, for $\bar{g}=440$ :

$$
\Delta \mathrm{w}=0.43
$$


This is a very significant change, and for $90^{\circ}$ Bloch walls all the contrast effects can be attributed to the magnetostriction.

\section{Influence of the Field of the Objective Lens}

on the Spin Arrangements

Measurements of Bozorth et al. ${ }^{41}$ have shown that in cobalt ferrites the magnetization vectors, $\bar{M}$, inside the domains have a strong preference for the $\langle 100\rangle$ directions. This was also pointed out by Hoselitz ${ }^{11}$ who remarked that the addition of cobalt ions to a ferrite spinel should strongly increase the anisotropy in favor of magnetic ordering in the $(100\rangle$ directions. Since all the foils examined in the electron microscope were well above $1500 \AA$ thick only Bloch walls were to be expected. The presence of the magnetic field of the objective lens (7000 Gauss at the specimen) will have a strong influence on the arrangements of the magnetization vectors in a thin film observed in the electron microscope. The position of the specimen in the field of the objective lens is shown schematically in Fig. 20. The magnetic energy density, $U$, of a piece of ferrite with a magnetization $\bar{M}$ per unit volume 43 , when placed in the field $\bar{H}$ obj of the objective lens:

$$
\mathrm{U}=-\overline{\mathrm{M}} \cdot \overline{\mathrm{H}}_{\mathrm{obj}}
$$

$U$ is the minimum when $\bar{M}$ is parallel to $\overline{\mathrm{H}}_{\mathrm{ob} . j}$. The preferred arrangement of the magnetization vectors in the different ferrimagnetic domains will be such that the components of $\overline{\mathrm{M}}$ which are anti-parallel to $\overline{\mathrm{H}}_{\text {obj }}$ will be minimal. For this reason $90^{\circ}$ walls are preferred over $180^{\circ}$ walls. For a (III) foil the $90^{\circ}$ domain structure then consists of a combination of magnetization vectors as shown in 'ig. 21. For such a foil stress free domain walls have to be in (110), (101) or (011) planes, as can be derived 
by considering Fig. 19. It should also be noted that, since the magnetic field of the objective lens. is particluarly strong in a high voltage microscope ( $7 \mathrm{k}$ Gauss at specimen) it may be possible that the direction of magnetization in the different ferrimagnetic domains is somewhat deviated from $\langle 100\rangle$ directions.

The above considerations account for the facts that only $90^{\circ}$ walls were observed in the specimens, and that in some toils urusual, non-equ1l1brium configurations were present. These observations are described in more detail below.

\section{Observations of Ferrimagnetic Domain Walls}

In most specimens ferrimagnetic domain walls were observed. A special difficulty was present in the determination of lie urientation of the domain walls planes, since they move when the specimen was tilted. In Fig. 22, however, the domain wall plane could be determined. This micrograph shows contrast from two overlapping domain walls in a wedge shaped foil. The orientation of the walls followed from a geometrical analysis: In the (323) surface of the foil, the trace of the wall is in the [101] direction. This gives as possibilities either a (010) plane for a $180^{\circ}$ wall or a (101) for a $90^{\circ}$ wall. The projected width of the domain wall, measured along the loth extinction contour, is $22000 \AA$. In this image $\bar{g}=404$ and with ${ }^{404}$ almost equal to zero, $\xi_{440}$ can be calculated to be about $800 \mathrm{~A} .30$ At the loth extinction contour the foil would then be about $8000 \mathrm{~A}$ thick. The geometry is shown in a stereographic projection, Fig. 23. The angle between the direction of measurement in the wall and the direction of measurement on the surface is either $56^{\circ}$ for a $(010)$ 
wall, or $18^{\circ}$ for a (101) wall. The foil thicknesses derived from these angles was correspondingly $34000 \AA^{\circ}$ or $7500 \AA$. It was clear that in this case the ferrimagnetic domain wall must have been on the (101) plane, indicating that the magnetization vectors in the adjacent domains were indeed at. $90^{\circ}$ to each other. Further information about the direction of $\overline{\mathrm{M}}$ came from the nature of the fringe contrast at the domain boundaries. Contrast from coherent domain boundaries has been discussed by Gevers et al. 44 Since the domain wall plane was known, the top and the bottom of the wall could be indicated, and from the nature of the extreme fringes, the change in $\bar{s}$ across the boundary was found. In Fig. 24 the direction of $\mathrm{M}$ is indicated so as to be in agreement with the observed fringe contrast. Note that the nature of the extreme fringes is opposite for the two walls. This was just as expected, since the sign of $\Delta \bar{s}$ is opposite upon crossing the different walls, see Fig. 25. Note also that $\bar{M}$ in the different domains always has a component paraliel to $\overline{\mathrm{H}}_{\mathrm{obj}}$ as followed from the discussion in the previous section. Most of the observed domain walls had a sawtooth configuration. This is especially clear in Fig. 26. These configurations arise when the surface of the foil is not an exact plane with low Miller indices. ${ }^{45}$ Note that the geometry of the arrangement of the walls indicates the presence of three different directions of $\bar{M}$. Such configurations are only found when the foil is in a near (111) orientation.

The mobility of the domain walls is illustrated in Fig. $27 a$ and $\mathrm{b}$. These micrographe also intirate hnw the domains grow. Most commonly observed is a domain growth as depicted below: 

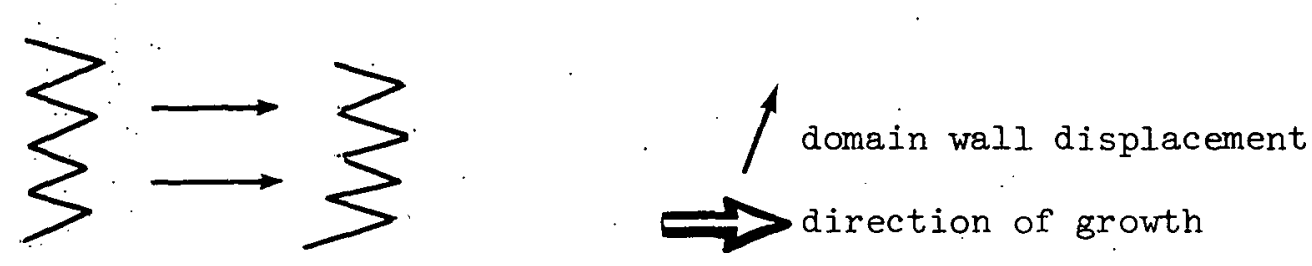

Note that in Fig. 28b, the zigzaging in the lower part of the image has a pitch as low as 0.25 micron. For such rapid zigzaging, the walls cannot be resolved directly, since they interfere with each other. in the image formation; their presence can, however, be derived from the oscillation of the extinction contour. These observations evidence that zigzag walls can exist on a scale much finer than has been observed so far with the most sophisticated Bitter techniques. It is interesting to note that in this case the domain wall motion is nearly perpendicular to the direction of growth. Borrowing the terminology of the dislocation theory one might say that this wall would be of the screw type. The equivalent of an edge dislocation would then be when the domain growth is parallel to the domain wall displacement, as is shown below.
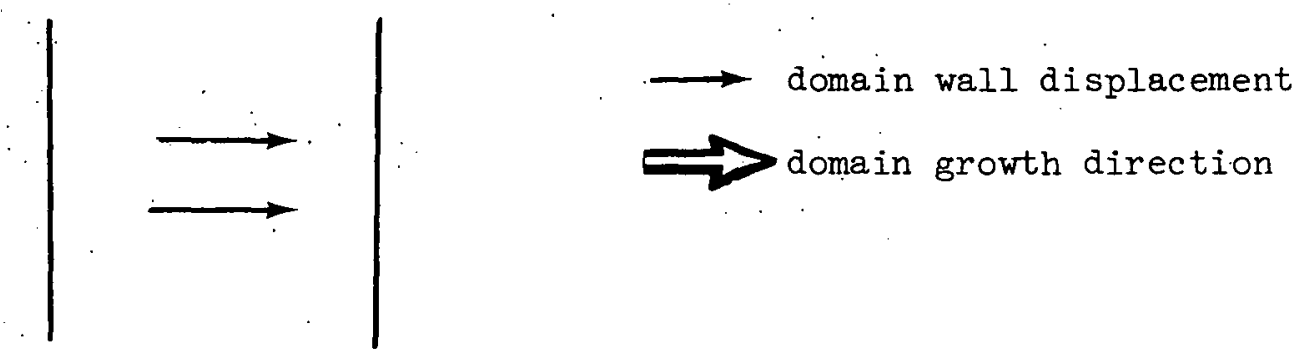

Doubling of Kikuchi lines due to the lattice rotation across the domain wall was observed. In Fig. 28, a selected area diffraction pattern of an area $\mathrm{A}$ inside a domain, and an area B containing a Bloch wall are compared. The brighter circular area B corresponds to the area 
isolated by the field limiting aperture. The double and parallel Kikuchi lines for the area $B$, evidence that the crystal rotation is about an axis perpendicular to the wall trace, and nearly in the plane of the foil. For such a configuration, the magnetization vector rotates over only $90^{\circ}$, which is the $\pi / 2$ wall orientation of the lowest magnetic energy. Such a configuration can only exist in thin foils where the lattice rotation can be accomodated by buckling. If the specimen increases in thickness, the elastic energy associated with a $\pi / 2$ domain wall of the type observed here would quickly become very high.

Figure 29 shows some domains in the 440 dark field. Note that the walls have a sawtooth configuration near the foil edges." The edges were also observed to be the nucleation sites of new domains, when the foil was tilted. The domain walls do not seem to be confined to the same plane, over large distances, although this is elastically not favorable. The buckling of the domain walls may have been due to imposed elastic stresses that can arise in a specimen mounted for observation, to surface orientation dependent demagnetization fields, or to long range composition fluctuations. 45

The magnetic domain wall images have a line of symmetry in wedged crystals: The position of this line of symmetry was called the "diffraction center" by Gevers et al. 44 The diffraction center is located in the foil at a depth determined by

$$
\sigma_{1} t_{1}=\sigma_{2} t_{2}
$$


where the parameter $\sigma_{i}$ is more or less proportional to the deviation parameter $s_{1}$ in the top a part of the foil. $\sigma_{2}$ is similarly related to $s_{2}$ in the bottom part of the foil.

$$
\begin{aligned}
t_{1}= & \text { distance from the top surface to domain wall, along the } \\
& \text { foil normal } \\
t_{2}= & \text { distance frum lie domain wall to bottom sirfers. }
\end{aligned}
$$

When the crystal is rotated around the symmetrical position, $s_{1}=s_{2}$, the diffraction center moves in accordance with Eq. 11. This rotation can also be realized by elastically bending the foil. This allows the examination of the effect of an elastic stress on the domain wall configuration:

The effect of a mechanical stress on the domain wall configuration is shown in Fig. 30. Inspection of the position of the diffraction center shows that the foil is elastically bent around an axis that is perpendicular to the wedge fringes. The tetragonality induced by the magnetic ordering causes a slight rotation across the coherent domain wall, as was 'described earlier. The sense of' the rotation deperms vin the sign of $\Delta s$. The bending of the foil was due to mechanical constraints imposed when the specimen was clamped between the mounting grids, or maybe also to contamination stresses. The segments that tended lu wurk against mechanical constraints were ndrrower than the other ones, so that the total elastic energy was lowered. This caused the succession of wide and narrow domains. 
In summary:

(a). Magnetostriction contributes most to the electron imaging of $90^{\circ}$ ferrimagnetic domain walls in cobalt ferrites. The contribution of the Lorentz effect to these images can be neglected.

(b). The field of the objective lens was 7 kilo Gauss at the specimen. Due to this high field; only $90^{\circ}$ Bloch walls could occur in the foil, and then only when the foil normal was nearly contained in $\{110\}$. planes.

(c). The fringe contrast of the $90^{\circ}$ walls was in very close agreement with the one predicted by the theory. This confirmed that the domains were also tetragonal twins, where the tetragonality was caused by the magnetostriction. From the nature of the fringe contstant, and the direction of $\overline{\mathrm{H}}_{\mathrm{obj}}$; the magnetization vectors could be found unambiquously.

(d). The motion of zigzag walls through the specimen was shown. The pitch of the zigzaging changed during the displacement of the wall, possibly due to interaction with the local foil geometry. A pitch as small as 0.25 micron was observed. This evidenced that zigzaging can occur on a much finer scale than observed earlier with high resolution Bitter techniques.

(e). Doubling of Kikuchi lines was observed in diffraction patterns taken from areas that contaimed $90^{\circ}$ Bloch walls. The doubling of the Kilkucki lines was due to the lattice rotation that accompanies tetragonal twinning.

(f). Wall configurations were observed that cannot exist in thick specimens, due to the high elastic energy that would be associated with $14,9 \%$ 
(g). Elastic bending of the foil produced a succession of wide and narrow domains. This effect was due to magnetostriction, and the possibility of lowering the elastic energy of the system by chaning the wall configuration.

\section{Phase Transformations}

Phase transformations were studied in cobalt feiril.th l.hH. wore transformed in two different environments: in air, and in the atmosphere of the electron microscope (hot stage). The hot stage experiments will be discussed first.

1. Transformations at Reduced Oxygen Partial Pressure: Hot Stage Experiments

Foils of cobalt ferrite with a Co/Fe ratio between 0.54 and 0.16 , were transformed in the hot stage of the electron microscope. It turned out that in this composition interval there was no significant difference in the morphology and nature of the hot stage transformations. For this reason, these experiments will not be discussed seperately. The composition of the microscope atmosphere, at the temperatures at which the hot stage experiments were carried out, was strongly reducing, and as the heating proceeded, the specimen lost oxygen to its surroundings. The details of the reduction were discussed earlier. As a consequence, so long as equililrium has not been established, a dynamic situation prevails in the specimen. The excess cations created at the decomposing crystal surface diffuse into the specimen, so that a time dependent cation cxcess gradient is present. The local deviation from stochiometry is the main driving force of the phase transformations. Evidence of this may be seen in the fact that foils heated in air at the same temperature, did not contain any precipitates, even after $60 \mathrm{~min}$. of heating. 
During the hot stages transformations the foil may simultaneously exhibit different phase transformations at different depths. The images of the transforming foils will have to be interpreted bearing this situation in mind. Somewhat arbitrarily, the transformations have been divided into three stages: the early, intermediate, and final stage. The purpose of 'this distinction' was mainly to facilitate the presentation.

It needs to be pointed out that although the hot stage was calibrated, still large uncertainties existed in the actual temperature at which the annealings in the microscope were carried out. For this reason, the hot stage work can only be considered as giving rather approximative quantitative information. The: temperature uncertainties prohibited measurements of transformation kinetics.

\section{The Early Stages}

When the precipitates were small, and the reactions proceeded rapidly, the transformation çould be said to be in the early stage. The duration of this stage depended strongly on the temperature at which the transformation was carried out, and on the judgement of the observer. The observations were made on foils that had not been electron irradiated prior to the transformation, although this precaution seemed to make little difference. It should be pointed out that care should be exercised in. the examination of phase transformations in the high voltage electron microscope, since $\mathrm{Nes}^{46}$ has recently observed electron radiation induced. precipitation. in high purity silicon. For short transformation times the. observations were made after the specimen had cooled from the transformation temperature. For these brief heatings, the effective annealing time and 
temperature had to be derived from heating and cooling curves of the hot stage, Fig. 3.

The aspect of a foil transformed for about $3 \mathrm{~min}$ at $500^{\circ} \mathrm{C}$, is shown in Fig. 31. Inspection of this image reveals that there are two different types of precipitates. For the present, the larger precipitates will be called Type 1 , while the very small ones are 'lype '2.

Of particular interest "is the location in the foil of these apparently different types of precipitates, their identification and orientation relationships, and finally, the nature of the phase transformations involved. These points are discussed in succession below.

a. Location of the precipitates in the foil

It is possible to locate the precipitates in the foil, by taking two images of the same area, with approximately the same diffracting conditions, but such that there is a difference of about $10^{\circ}$ in the specimen tilt between the two images. The two images then cunstitute a stereo pair. The axis of tilt should be perpendicular to the operating reflection. 30 It was found that an angle of tilt of about $5^{\circ}$ was better suited for the achievement of the stereo effect, possibly because of the high defect density, and the large foil thickness (compared to the foils suitable for $100 \mathrm{kV}$ clcctron mlerosoopy). A stereo pair taken under these conditions is shown in Fig. 32. Study of this pair with stereo viewers showed that the transformations were taking place at different levels in the specimens. The Type 1 precipitates, were actually located very near or on the top and the bottom surface. The Type 2 precipitates were located deeper inside the foil, and were separated from the surfacc reaction zone by an apparently precipitate free region. The geometry of 
the reaction zones is shown in Fig. 33. The Type 1 precipitates have an apparent size of about $400 \AA$, and are separated about $1500 \AA$ on the average. The Type 2 precipitates in the inner reaction zone have a diameter of about 30 to $40 \AA$ and seem to be separated in the average approximately $150 \AA$. The estimated precipitate density in the inner reaction zone was then about $3 \times 10^{17} \mathrm{~cm}^{-3}$. This was quite high. With these approximate data a rough estimate of the volume transformed, was only $1 \%$.

It is worth mentioning that near the very edge of the foil, where it is less than $1000^{\circ}$ thick no precipitates of Type 2 were observed. This again showed the merit of high voltage transmission electron microscopy. In the thin foils that are to be used in $100 \mathrm{kV}$ instruments, the Type 2 precipitation mode might be missed entirely.

\section{b. Identification, and orientation relationships of the precipitates}

With the conditions prevailing during the hot stage transformations, only oxides could form that had an anion to cation ratio that was smaller, or at most equal to that of the original spinel matrix. This greatly facilitated the identification of the precipitates by means of electron diffraction, and out of the large number of oxides that could form in the Co-Fe-0 system, only a few had to be considered:

The identification of the different precipitates was carried out by examining numerous reflections in dark field. It should be noted that the use of a 5 micron objective aperture was mandatory, since most reflections of one kind of precipitate were usually very near others. The procedure in such a complicated case, where several phases are present at the same time, is to postulate the presence of a certain type of precipitate, and then to verify this postulate by constructing the corresponding 
diffraction pattern. If the reflections in the actual diffraction pattern agree with the postulates, then final identification comes from imaging every individual diffraction spot. This rather laboreous procedure was adopted.

From a scrutiny of diffraction patterns, and concurrent dark field imaging it appeared that the Type 1 precipitales were a mixture of cubic and hexagonal cobalt metal. The Type 2 precipitates were a cobaltwüstite phase. These findings are examined in more detail below.

The actual diffraction pattern (enlarged to about 10 times its original size) of a 100 foil transformed for $3 \mathrm{~min}$ : at $500^{\circ} \mathrm{C}$ in the hot stage, is shown in Fig. 34a. The solution is shown in Fig.34b. The circular area of higher intensity around $h_{1}$ in Fig. $34 a$, is an image of the 5 micron objective aperture. Reflections of the hexagonal cobalt are marked $h$. They are all $10 \bar{I}$ type reflections. They have not been indexed, since they cannot be labelied unambiguously. The cobalt-wütite reflections could not be resolved at the lower order reflections. For higher order reflections though they could indeed be separated, as is shown in Fig. 35. The arrows in this figure point towards the center of the diffraction pattern. The sodium chloride type phase is the only one in this system that has a latticc parameter that is elightly larger than half the lattice parameter of the spinel matrix, fitting these diffraction results. It has not been possible to ever eslimate the Co/Fe ratio in this cobalt-wústite phase, since the lattice parameters of $\mathrm{CoO}$ and FeO differ by only $1 \%$.

In Fig. 36 the various precipitates are imaged. The diffraction spots that were isolated with the objective aperture, and hence were 
contributing to the dark field images, are shown in the inserts. Although a matrix reflection is included in Fig. 36b, matrix contrast is low. This was achieved by placing the $040 \mathrm{~m}$ on the reflecting sphere, and then imaging the $040 \mathrm{~m}$ cluster of reflections. Under those conditions good image contrast can be obtained from small defects. 'I'his method is similar to the weak beam method that has been used for separating close partial dislocations. 34 In Fig: 36 c only the Type 1 precipitates shown. Note that the "satelites" to the main reflections are really reflections from the metallic cobalt precipitates. They do not arise from a particular spacial arrangement of the cobalt-wústite precipitates; otherwise, these precipitates would also be imaged. Figure $36 \mathrm{~d}$ again shows the cobalt metal phase, this time imaged with a $10 \bar{I}_{1}$ type reflection, $h_{1}$. Note that many precipitates in this dark field image have flat sides that are parallel to the $(110)$ directions of the matrix. In Fig. 37 another area in a 100 foil treated as above, is shown. In Fig. 37b, the "weak beam" techique was used once more to image the cobalt-wistite precipitales with their 020 reflection. Note again that the precipitates are randomly distributed.

The cobalt-wüstite phase was topotactic with the spinel matrix. Since the oxygen sublattice parameter difference was only $1.5 \%$ the precipit.a.tes were likely to be completely coherent with the matrix. The oxygen sublattice was then continuous throughout the two phase crystal.

The orientation relationship between the cubic cobalt metal, $c$, and the matrix $m$, was also simple:

$$
\begin{aligned}
& (001)_{\ldots} . \| \quad(001) \\
& {[100]_{c} \|[100]_{m}}
\end{aligned}
$$


The orientation relationship between the hexagonal cobalt and the matrix was found to be:

$$
\begin{aligned}
& (0001)_{h} \|(111)_{m} \\
& {[21 \overline{1} 0]_{h} \|[110]_{m}}
\end{aligned}
$$

This relationship was also observed between cobalt spinel that forms on hexagonal cobalt, when the metal was oxidized. 47

Since the cubic cobalt had the ame orientation as the matrix, it followed that

$$
\begin{aligned}
& (0001)_{h} \|(111)_{c} \\
& {[2 \overline{1} \overline{1} 0]_{h} \|[110]_{c}}
\end{aligned}
$$

This orientation relationship was also found when cubic cobalt transformed to its hexagonal form, or conversely. The hexagonal cobalt. was then most likely formed from the cubic cobalt, when the crystal was cooled after the hot stage annealing. The hexagonal cobalt should form on $\{111\}$ planes of the cubic metal. Evidence of this was found in Fig. 36d: the flat sides of the precipitates coincide with the trace of $\{111\}$ planes in the (.001) foil plane. Better information about the structure of the cobalt precipitates was obtained at a later transformation stage when the precipitates were large. The presence of hexagonal and cubic cobalt at room temperature indicated that, according to the cobalt-iron phase diagram, ${ }^{48}$ the metallic precipitates contained less than $5 \%$ iron.

\section{c. Nature of the phase transformation}

Phase transformations is one of the most intensely studied areas in materials science. The current effort is largely focused on the early stages. In spite of the enormous amount of work that has been done in the past, it was only very recently that some unified views emerged concerning 
the early stages of decomposition. 49 All nucleation phenomena are describable in terms of composition and displacement fluetuation models which are more easily treated in their Fourier space. At the same time it gets more difficult to make a clear distinction between one kind of transformation or the other, especially when the supersaturations are large. Beyond the nucleation stage the understanding of phase transformations seems considerably better, and detailed treatments can be found in Christian's book. 50

Recently, Fine ${ }^{51}$ has given a review of decomposition in non-metallic solid solutions. While homogeneous transformations are very rare in metallic systems, examples may be found in oxide ceramics when transformations occur by transport of the cation interstitials This is e.g. the case when magnesioferrite spinel precipitates in MgO that is supersaturated with iron. 52 In our case the situation was more or less reversed, since now a cobalt-wustite phase precipitated in a spinel matrix. The observed density of the precipitates was for the present treatment about a factor of 10 higher (that is $3 \times 10^{17}$ per $\mathrm{cm}^{3}$, see earlier) than what was found for the magnesioferrites precipitates in MgO. Also no preferred precipitation at dislocations was observed, as is clear from an examination of Fig. 38. The reason for this truely homogeneous transformation was possibly that the cation diffusion rates were considerably enhanced by the low oxygen partial pressure prevailing during the transformations. Th1s was discussed in the section on point defect chemistry. Enhanced diffusion at dislocations is likely to be of small significance when the diffusion rates in the bulk are high. It was also pointed out in the previous section that the cobalt-wústite particles precipitate ran- 
domly, so that a spinodal like decomposition has to be ruled out. Spinodal decomposition with $\partial^{2} G / \partial c^{2} \leq 0$ is actually very unlikely, since the precipitate has a different crystal structure than the parent phase.

It is difficult to make any reliable conclusions regarding the nature of the dernmpnsition leading to the formation of metallic cobalt, since this transformation occurred on the surface of the specimen. These metallic particles were most likely formed through a heterogeneous decomposition since the difference between the two phases involved was more drastic. Note that foil edges and surface steps got preferentially decorated, as is shown in Fig. $39 a$ and B. Steps such as shown in Fig. 39b were frequently found on the surfaces of specimens that were accidentially cleaved. Part of this decoration effect was probably due to faster loss of oxygen at these geometrically more favorable sites. "Locally the reaction would then proceed at a somewhat higher rate.

Note that very high coercive forces should accompany a microstructure such as the one found in the early stages. The coercive force should be especially high below the Neel temperature of the cobalt-wístite phase.

\section{The Intermediate Stages}

As the heating continued, the transformations proceeded to the intermediate stage. It was expected that the precipitates that were nucleated in the early stages would continued to grow, except where the changing anjon cation ratio had changed sufficiently so as to locally cause a different phase relation. Thus, for the present geometry further reduction of the foil should lead to continued growth of the surface metallic phase, and to shrinkage of the spacial extent of the inner reaction zone that 
was containing the cobalt-wustite precipitates. A clear impression of what was going on in the intermediate stages came from the observation of transforming, wedged crystals. In such a crystal, the shrinkage of the inner reaction zone should manifest itself in the images as a receding boundary. At the moving boundary between the precipitate free zone and the inner reaction region, the ( $\mathrm{Co}, \mathrm{Fe}) \mathrm{O}$ particles should shrink, and eventually dissolve completely. Inside the reaction zone, these particles should continue to grow. All of this can be observed in the sequence Fig. 40a to d. In this series a. wedge shaped foil was imaged while transforming, after the early stages had passed. The foils were heated for $120 \mathrm{~min}$. before the sequence was started. This allowed the hot stage to get stable, so that the observations could be made at temperature. The numbers in the micrographs refer to the ellapsed time in minutes. Note that the cobalt-wústite particles show double arc strain contrast. Such a particle has been marked with a black arrow. As the transformation proceeds, and the inner reaction zone shrinks, this precipitate shrinks In size, and finally dissolves completely after $140 \mathrm{~min}$. Other cobaltwistite precipitates that were not so close to the moving boundary may be seen to have increased in size somewhat. An example of such a precipitate is marked with a white arrow. The precipitate density could be estimated from the spacing of the particles, and is found to be about $2 \times 10^{16}$ per $\mathrm{cm}^{3}$. This is about an order of magnitude less than the precipitate densities that was observed in the early stages. At the same time compared to the early stages, the precipitate size has clearly increased. The size could be estimated from the strain contrast, 21 and was found to be on the average $150 \AA$ in diameter. At this time then the volume fraction of the cobalt- 
wüstite phase in the inner reaction region is about 5\%. The volume fraction of the transformed material would, in the middle of the inner reaction region, still be increasing because the crystal was further reduced when the heating in the electron microscope atmosphere was continued.

The ctrain contrast of the cobalt-wistite particles is examiner in more detail in Fig. 4l. These images typically result from the strain field of coherent particles'2l so that it must be concluded that the cobaltwústite particles are completely coherent with the matrix spinel, at least up to this size. At all times the line of no contrast is perpendicular to the operating reflection, so that the matrix strain fields surrounding the precipitates are spherically symmetrical. The strain field images 'are not black-whtte Lobed, since the preclpitates are not close to the surface. For this reason, the sign of the strain field could not be found from the examination of electron images. However, the particles were identified earlier, as cobalt-wístite and since this cubic phase has an oxygen sublattice parameter that is slightly larger than that of the matrix, the strain fields must be compressive. Note also that the inner reaction region is not separated from the precipitate free zone by a sharp and distinct interface. This is because the spinel matrix only changes composition when the $(\mathrm{Co}, \mathrm{Fe}) 0$ precipitates, and does itself not change crystal structure.

The large precipitates in Fig. 41., were identified in the previous section as a highly cobalt rich metallic phase. In a simplified way the decomposition in the reducing atmosphere of the microscope of a cobalt ferrite into highly cobalt rich metal and highly iron rich spinel can be 
written as follows:

$$
3 \cdot \mathrm{CoFee}_{2} \mathrm{O}_{4}=3 \mathrm{Co}(\mathrm{s})+2 \mathrm{Fe}_{3} \mathrm{O}_{4}(\mathrm{~s})+2 \mathrm{O}_{2} \text { (gas) } \ldots
$$

All the ferrites spinels have about the same molar volume; since their lattice parameters are very close. The volume of the three moles of cobalt that are formed when three moles of cobalt ferrite are decomposed according to the above scheme, does not compensate for the loss of 1 mole of ferrite, so that an over all reduction of about $25 \%$ in volume occurs upon complete transformation. This significant volume reduction was reflected in the transforming foils by the formation of holes in the matrix spinel around the growing metal particles. The effect was especially pronounced at the foil edge, where the surface to volume ratio was particularly high, (see Fig. 40). The metal precipitates remained attached to the matrix, and actually did move around somewhat so as to keep in physical contact. This motion, which was more or less determined by the local geometry, slowly destroyed the orinetation relationship between, the matrix and the metallic precipitates.

\section{The Later Stages}

In the later stages the foil was near equilibrium with the surrounding atmosphere, and only grain growth (which appeared to be a very slow process here) occurred. With the prevailing atmosphere and heating conditions, the foil consisted of a very highly cobalt rich metallic phase; and a spinel phase which was consequently very high in iron content. The foil thickness was a strong factor in determining how rapidly the specimen approached the later stages. This is illustrated in Fig. 42, which is an image of a wedge shaped foil after heating for $150 \mathrm{~min}$. at $550^{\circ} \mathrm{C}$. The foil edge is at the left. Note that in the thicker 
part of the foil, at the right, the coherent cobalt-wustite particles are still present in the inner reaction zone. The diffraction pattern was taken from the entire area. The deterioration of a definite orientation relationship between the matrix and the metallic precipitates is evident form this diffraction pattern, since many non-systematic precipitate reflections are present. The internal structure of the metalilc phuse is shown in l'ig. 43. Occusionally it was possible to isolate a single precipitate in the field limiting aperture, as was the case in Fig. 44. In a diffraction analysis of such a precipitate it was useful to apply a technique where pairs of diffraction patterns were examined, one focused and one defocused. 30 The analysis showed that this precipitate consisted of cubic and hexagonal cobalt, such that the $(0001)_{\text {hex }} \|(111)_{\text {cub }}$. This orientation relationship was already found in the early stages; where the internal structure of the Eurface precipitates could not be resolved adequately, and confirmed the earlier conclusion that the hexagonal cobalt was formed trom the culic phase upon cooling of the specimen after transformation. Another kind of substructure in the cobalt metal precipitates is shown in Fig. 45. In this micrograph, a highly cobalt rich precipitate is exactly in the 110 orientation: The diffraction pattern shows streaking perpendicular to the observed substructure. Ciose examination of lieste stied alrs ruveiled that they actually consist of a succession of discrete spots superimposed on the less intense streaks. The spacing of the spots in the diffraction pattern corresponded to the substructure spacing in the precipitate. A further analysis showed that origin of the diffraction effect is due to the almost periodic micro-twinning or faulting of the cubic cobalt prccipitate, with a periodicity of about $45^{\circ}$. 
4. Hot Stage Transformations in Less Reducing Atmospheres

As was mentioned in the experimental section, the composition of the microscope atmosphere did not remain constant over extended periods. Whether or not free metal was thermodynamically possible at: a certain temperature, depended critically on the excess hydrogen present. This excess was variable, and in fact, occasionally excess oxygen was found instead. This excess oxygen partial pressure was usually in the order of $10^{-7}$ or $10^{-8}$ Torr, and free metal should not be formed in that case. It was then to be expected, that growth and coarsening of the wustite type precipitateswould just continue as the transformation proceeded. This was indeed the case, as is shown in Fig. 46 for a foil that was transformed for $10 \mathrm{~min}$. at about $650^{\circ} \mathrm{C}$ in an effective oxygen partial pressure of about $2 \times 10^{-8}$ Torr. It is interesting to note that the precipitates seemed to be periodically arranged. Often this periodic arrangement is interpreted as evidence of a spinodal decomposition. However, periodic arrangements are not necessarily conclusive evidence of spinodal decomposition, as was shown in the work of Ardell and Nicholson. ${ }^{53}$ They found modulated arrangements of precipitates in the Ni-Al system when the transformation was in the coarsening stage, but random precipitates in the early stages. The reason for the development of periodic arrangements was due to the elastic interactions between the growing precipitates. In the present case, it was observed that the (Co,Fe)o particles initially formed randomly. The apparent periodic arrangement that was observed in Fig. 46 must then originate from elastic interactions upon coarsening, jusl as was the case for the $\gamma^{\prime}$ precipitates in the Ni-Al system; they cannot be considered as evidence of a spinodal decomposition. 
In summary:

(a). Hot stage transformations are of a highly dynamic nature. The transformations are due to the loss of oxygen from the sample surface. The excess cations formed in this way diffuse into the sample, creating a time dependent cation/anion gradient from the surface.

(b) In thè eâr ly stagés a very hıgh densıty of (C'o, f'e)U precipitates forms. The decomposition is homogeneous, and inolves mainly the transport of cations from the crystal surface.

(c). Cobalt metal, containing less than 5 at.\% $\mathrm{Fe}$, is formed at the crystal surface. After cooling from the transformation temperature, hexagonal cobalt is found. The hexagonal cobalt forms from the cubic one by a twinning or faulting reaction.

(d). All orientation relationships are simple.

(e). Examination of transtorming wedged crystals show the existence of the ditf'erent reaction zones. Spherically symmetrical strain contrast was observed at the ( $\mathrm{Co}, \mathrm{Fe}) 0$ precipitates.

(f). The growing metal grains slowly destroy their original orientation relationship with the matrix, since they remain in physical contact with the matrix that is consumed.

(g). In the later stages the interinal shruclure of the cobalt grains was examined in more detail. In some cases quasi-periodical twinning or faulting is observed, giving rise to long streaks in the diffraction patterns.

(h) Very high coercive forces are expected for thin foils that are treated to contain a two phase structure as is found in the early stages. 
(i) For hot stage transformation in partial oxygen pressure of $210^{-8}$ Torr, at $650^{\circ} \mathrm{C}$, no free metal forms and the (Co,Fe) phase continues to grow. In this coarsening stage the precipitates are arranged in a modulated structure. The modulated structure is a result of strain interactions, and does not result from spinodal decomposition.

\section{E. Transformations In Air}

1. Annealings at $670^{\circ} \mathrm{C}$

Single crystalline specimens with a Co/Fe from: 0.54 to 0.76 kept at $670^{\circ} \mathrm{C}$ for up to two weeks, failed to show precipitates. Numerous foils were examined but there was no trace of a eutectoid decomposition of the matrix spinel into hematite and a very cobalt rich spine, as Fig. la might suggest.

\section{Annealings at $720^{\circ} \mathrm{C}$}

Crystals with a composition of $\mathrm{Co}_{1.29} \mathrm{Fe}_{1.71} \mathrm{O}_{4}$ were heated for several days at $720^{\circ} \mathrm{C}$. The phase diagram, Fig. la suggests that a decomposition into two spinels should occur. While Takahashi and Fine ${ }^{54}$ have reported a spinodal decomposition for higher cobalt contents in this miscibility gap, no precipitation was observed for crystals with a Co/Fe ratio of 0.76 . Single crystals with a higher. Co/Fe were unfortunately not availab.?

In view of the above observations, it seemed that the phase diagram suggested by Aukrust and Muan, 6 is in error. The recent observations of Takahashi and Fine 54 also confirmed this, and all the evidence now. indicates that the phase diagram reported by Robin ${ }^{\top}$ is the more correct one.

\section{Annealings at $1230^{\circ} \mathrm{C}$}

Crystals with a Co/Fe ratio of 0.76 were annealed for a series of times at $1230^{\circ} \mathrm{C}$. For this temperature the phase diagram shows equilibrium 
between the spinel $\mathrm{Co}_{1.18} \mathrm{Fe}_{1.82} \mathrm{O}_{4}$, and the sodium chloride type ( $\mathrm{Co}_{0.82}$ $\mathrm{Fe}_{0.18}$ )0. Negelecting the oxygen non-stochiometry, the transformation reaction reads, according to the lever rule,

$$
\begin{aligned}
\mathrm{Co}_{1.29} \mathrm{Fe}_{1.71} \mathrm{O}_{4} & \rightarrow 0.26\left(\mathrm{Co}_{0.82} \mathrm{Fe}_{0.18}\right) 0+0.91\left(\mathrm{Co}_{1.18} \mathrm{Fe}_{1.82}\right) \mathrm{O}_{4} \\
\ldots & +0.10 \mathrm{O}_{2}(\mathrm{~g}) \uparrow
\end{aligned}
$$

Oxygen non-stochiometry, which is very likely to occur at this high transformation temperature, would slightly alter the amount of oxygen gas formed per mole of spinel that is transformed. Equation 13 shows that after complete transformation the (C⿺,Fe) o precipitates occupy about $7 \mathrm{vol} . \%$ of the crystal. Since the transformation reactions were of the same nature as in the hot stage experiments, certain similarities might be anticipated. The cation mobility could be estimated from the data of Schmaltzreid et al. 14,15 At $1230^{\circ} \mathrm{C}$, the diffusion coefficient of cobalt would be about $10^{-9} \mathrm{~cm}^{2} \mathrm{scc}^{-1}$, so that after $100 \mathrm{sec}$, lle mean dirfusiun distance of this cation would be about $1 \mathrm{~mm}$, so that, as was the case in the hot stage transformations, a reaction zone should develop from the specimen surface. Again, the transformation depended on the long range cation diffusion, since at all temperatures oxygen is the slower diffusing component. In view of this gradient phenomenon, it was difficult to measure precipitate growth rates, since the material from which the foil was eventually prepared could not be located accurately with respect to the surface of the bulk sample. Still, the general trend of size increase for longer isothermal annealing times should be preserved.

a. 10 minutes at $1230^{\circ} \mathrm{C}$ in air

(Co, $\mathrm{Fe}$ ) 0 precipitates formed in $\mathrm{Co}_{1.29} \mathrm{Fe}_{1.71} \mathrm{O}_{4}$, upon heating for 10 min. at $1230^{\circ} \mathrm{C}$ in air. Some of these precipitates are shown in Fig. 48, 
with two different operating reflections. Weak structure factor contrast observed when $\bar{g} .=800$, indicated that the precipitates where in fact orthorhombic prisms, bounded by $\{100\}$ planes. The images exhibited double arc contrast, which typically results from the strain field of coherent precipitates. Note that the line of no contrast, LC, takes on an "S" shape, when $\bar{g}=440$. In principle, the contrast effects should follow from the work of Sass et al. 55 These authors calculated the strain field electron images of coherent cuboid particles, for a limited number of diffracting conditions. The images of the orthorhombic prisms observed. here, should correspond to their images, when the precipitates are viewed with the long axis parallel to the electron beam. A precipitates satisfying this orientation is imaged under several diffracting conditions in Fig. 48. In the top left micrograph, the deviation from the exact Bragg condition is large, and the shape of the precipitate can be observed directly from the weak structure factor contrast. When the direction of the imaging reflection is not along a principal axis of the particles, the asymmetry of the strain contrast fringes is in agreement with the predictions of Sass et al. In particular, the appearance of a "notch" in the line of no contrast, LC, is confirmed. The shape of LC can now be understood more clearly, since the present observations, Fig. 47 and 48 , show that the peculiar images originate in the tendency of LC to follow the symmetry of the coherency fields, regardless of the direction of the operating reflection. Note that this is somewhat different from the strain contrast images of small prismatic loops. As was discussed by Thomas and Bell, 24 for prismatic loops, LC is perpendicular to the operating vector $\bar{g}$, when the angle between the direction of the Burgers vector of these 
loops, and $\bar{g}$ exceeds $15^{\circ}$.

The size of these coherent prisms varied in the same specimen; the largest coherent precipitate found had a size of $1200 \AA$.

In a foil of identically treated material, precipitates were observed as shown in Fig. 49. The precipitates now exhibit moire fringe contrast. They are actually arianged along a dislusation. A tentative cxplanation of this "string of beads" morphology may be as follows: The dislocation would end on the free surface of the treated crystal. The lower left part of this dislocation in Fig. 49 would be further away from the free specimen surface, so that the precipitates that were formed there were smaller than the ones closer to the surface.

Regardless of the location of the dislocation this observation evidenced that the wustite precipitates nucleated heterogeneously at $1230^{\circ} \mathrm{C}$ in air. The different morphologies, and the range in size also supported the idea that the transformations proceeded from the surface, and that a gradient in cation concentration was present, just as for the hot stage transformations.

b: 60 minutes in air at $1230^{\circ} \mathrm{C}$

Precipitates of ( $\mathrm{Co}, \mathrm{Fe}) 0$ found in cobalt ferrites that were transformed in this way are shown in Fig. 50. The precipitates lost perfect coherency with the matrix as is evident from the interface contrast. Isolated particles are more or less spherical. Other precipitates that are elongated or odd shaped probably formed by coalescence of several precipitates. Note also that the particles are connected by a few dislocations. The smallest precipitate that exhibited this type of interface contrast was about $1500 \AA$ in diameter. 
Inspection of the images of the matrix-precipitate interface reveals several features that need to be examined in more detail. This will be done in a later section.

c. Annealings for more than 60 minutes in air at $1230^{\circ} \mathrm{C}$

Longer annealing in air of the same material apparently did not lead to much change in the morphology of the precipitates. The same size and distribution was found in crystals that were transformed for 20 hours, or for six days at $1230^{\circ} \mathrm{C}$. An example of precipitates in cobalt ferrite $(\mathrm{Co} / \mathrm{Fe}=0.76)$ annealed for 20 hours, is shown in Fig. 51. The similarity with Fig. 50 is striking. The diffraction pattern from the area encircled in this micrograph, again shows the succession of single and double spots, confirming that the precipitate is $(\mathrm{Co}, \mathrm{Fe}) 0$.

A short summary of the transformation events, based on the observations, is: (1) coherent precipitates of ( $\mathrm{Co}, \mathrm{Fe}) 0$ are formed initially. The precipitates remain coherent up to a size of at least $1200 \AA$. No dislocations are found in conjunction with the coherent precipitates. (2) The precipitates lose coherency somewhere between 1200 and $1500 \mathrm{~A}$. (3) A small number of dislocations is invariably associated with the incoherent precipitates.

4. The Precipitate Interface

Interfaces in metallic systems have been studied e.g. by Weatherly and Nicholson. ${ }^{56}$ The interface between the (Co,Fe) 0 and cobalt ferrite matrix are now examined in the same light.

a. Moiré fringes and dislocation

For the examination of interfaces, some geometries are more favorable than others, and a large number of foils had to be prepared, until this 
favorable geometry was obtained. An extra difficulty was that the precipitates were so large that they often intersected the foil surface. In each case the actual geometry had to be determined.

In Fig. 52 precipitates that appeared to be completely inside the foil, are shown with two different operating reflections. The diffracting conditions are such that systematic sets of reflecliuns are operating, and so only the lowest order reflection, $g_{1}$, is marked. The fringcs are always perpendicular to the operating reflections, indicating that they are of the moire type. The detailed interpetation of the fringes is highly complicated, since their intensity (not their position) depends in a complex way on the depth of the interface in the foil. These and other characteristics of moiré fringes were discussed in great detail by Gevers. 57

The interface exhibited some other features in addition to moiré fringes, as is clear from Fig. 53. The geometry of this precipitates is drawn in perspective in Fig. 53b. The wedged surface of the foil intersects the precipitate in the shaded areas. Of interest are the "Iight patches" seemingly at the precipitate interface, as well as the particular fringe structure encircled and mared 2. The "light patches" are discussed later.

As is well known, semi-coherent interfases can be described in terms of misfit dislocations. Imaging of interface dislocations can be very difficult, especially if their spacing is small, and when moiré fringes are present simultaneously. The area marked 2 in Fig. 53a contains what might be interface dislocations. One of the simplest experiments to distinguish between interface dislocations and moiré fringes 
is to tilt the specimen over a small, angle in the electron microscope. so that the deviation parameter, $\bar{s}$, of the operating reflection is slightly changed. As Gevers 57 has pointed out, this should casue moiré fringes to shift. Images of dislocations don't exhibit this effect. Such a tilting experiment is shown in Fig. 54. The "light patches" can be considered as fiducial marks. Examination of these images shows that after a smali angle of tilt towards the exact Bragg condition, from $\bar{s}_{1}$ ' to $\bar{s}_{2}<\bar{s}_{1}$, the line images have not shifted. This confirms immediately that they are indeed dislocation images, and not moire fringes. Upon further tilting to $S_{3} \cong 0_{1}$, the moiré fringes appear. The imaged dislocations are parallel to the trace of the [110] direction, indicating that they have a strong edge component. Screws with b\| [110]. would be out of contrast with $g$ parallel to the [001] direction. The spacing of these misfit dislocations is about $340 \AA$. Assuming that the Burgers vector, of these dislocation is $1 / 2$ a matrix ( 110 gives for the present misfit a dislocation spacing of about $280 \AA$. If the dislocations would have a Burgers vector equal to $1 / 2$ a precipitate (110), then the dislocation spacing should be about $140 \AA$. The Burgers vector of the misfit dislocations is then most likely $1 / 2 a_{\text {matrix }}\langle 110\rangle$, with the "extra half plane" in the matrix (since the precipitate has the larger lattice parameter). It is quite common to find an interface misfit dislocation spacing that is somewhat larger than the theoretical one. Some reasons for this were given by Weatherly et al. 56 Another reason is quoted at the end of the next paragraph, and is due to the nature of the "light patches".

It is interesting to note that there still may be an easier way to check if dislocations are present at the interface. when strong moire fringe 
contrast is superimposed parallel to the dislocations. The technique would consist in a comparison of under and over focused images. When the focal length of the objective lens is changed, the moire fringes shift just enough to be detectable while observing on the screen. Dislocations do not. The different focusing behavior of moire fringes and dislocations io clcar in Fig. 55. Figure 55a c ohowo the moirc fringeo ouporimpocod on the dislocations, while Fig. 55d-f shows dislocations only. The degree of under and over focusing is the same in both sets. Note that the moiré fringes have half the spacing of the dislocations. It may be interesting to further study the difference in the spacial variation of these images. These effects, although not reported in the literature yet, are not examined further now.

\section{b. Micropores at the interface.}

The "light patches" at the interface were somewhat curious. It was not possible to obtain any diffraction from these defects, and their intensity always remained close to that of the surrounding background, regardless of the diffracting conditions, both in bright and dark field. Four different imaging conditions are shown in Fig. 56. It must be concluded that these "light patches" are in fact micoopores that have developed at the matrix -prooipitate interfaoe during the phase transformation. The pores are indeed exactly at the interface, as can be seen in Fig. 53 . Some pores, located just where the foil intersects the interface, are marked 1. No pores were ever found behind the matrix-precipitate interface, so that they must be migrating along with it. It is very doubtful that they would form upon cooling of the sample from the transformation temperature, since the cooling rates were several hundred degrees per 
minute. Migration of gas bubbles filled with fission gas, formed in copper irradiated with high doses of thermal neutrons, has been observed Barnes et al. ${ }^{58}$ indicating that the migration of such large defects was not extraordinary.

The formation of micropores during the transformation can only be explained by a mechanism which involves the transport of oxygen vacancies. A tentative mechanism of their formation would be as follows: In Eq. 13 the overall transformation reaction was given, and it was pointed out that the formation of the cobalt. -wïstite precipitates was controlled by the long range cation diffusion from the free surface of the crystal. At $1230^{\circ} \mathrm{C}$, however, this is a simplication, since the mobility of the oxygen vacancies . must be sufficient to participate, at least to a small extent, in the precipitation phenomenon. Part of the gradient in cation/anion ration must then be realized by the inward diffusion of oxygen vacancies. These oxygen vacancies, $V_{o x}$, are created at the surface of the heated crystal by reactions such as

$$
4 \mathrm{Fe}^{3+}+2 \mathrm{O}^{--}=0_{2}(\mathrm{gas})+2 \mathrm{~V}_{\mathrm{ox}}+4 \mathrm{Fe}^{2+}, \ldots
$$

Both the oxygen vacancies and the divalent cations diffuse toward the precipitate, where the phase transformation is continuing. The growing cobalt-wüstite precipitate consumes a large amount of divalent cations, but it can not incorporate the oxygen vacancies. As the precipitate increases in size, an excess of oxygen vacancnies would then build up in the crystal matrix, near the spinel - (Co,Fe)O interface. Since these oxides cannot support large deviations from stochiometry, the oxygen vacancies will have to cluster, probably first as vacancy loops, and later as 
actual. voids. The climb of these vacancy loops is limited by the requirement that they have to remain at the interface to accomodate the difference between matrix and precipitate lattice parameters. The dislocations and the micropores then act as effective oxygen vacancy sinks, allowing the further participation of oxygen in the transformation. Once a free suriace is present intcrnaliy, a roastion sirth is expresscd by Eq. 13 can take place. This would on the one hand increase the pore size somewhat, but on the other hand it would fill the pore with oxygen gas. When the equilibrium oxygen pressure has been reached, this reaction must cease. The presence of oxygen gas in the micropores might be inferred from their nearly spherical shape. The partial pressure of the oxygen gas should be sufficient to overcome the tendency of the pores to be bounded by low indices. crystallographic planes. 'l'his tendency is due to the anisotropy of the surface energy. A rough estimate of the pore volwae showed that they took up ahout $0.3 \%$ of. the volume of the precipitate. This was certainly not sufficient to compensate for the overall $2.5 \%$ decrease in volume that accompanied the transformation but it was direct evidence of the participation of the oxygen ions in the phase transformation. The presence of the micropores may partly account for the larger than calmulated spacing of the interface dislocations.

\section{c. Mechanism of the loss of coherency}

Loss of coherency of growing precipitates was discussed by Weatherly et al. 56,59 Several mechanisms have to be considered:

1. A punching mechanism in which one loop is emitted into the matrix; when one loop is being formed at the interface. 
2. Interface dislocations are supplied by sources in the matrix

3. Interface dislocations are supplied by sources inside the precipitate.

4. Dislocations are nucleated at the interface by collapse of point defect aggregates .

These four possibilities are now examined in the framework of the experimental observations.

Although there are examples of systems in which punching occurs; see e.g. Eikum and Thomas, 60 mechantsm 1 did not seem to be operating during the growth of the (Co,Fe)o precipitates. The dislocation density that was observed in the neighborhood of the large precipitates was too low to be in agreement with the punching model. Also, a minimum amount of misfit, about $4 \%$ between the lattice parameters of the precipitate and the matrix, seems to be necessary. 52 . In the present cases, this misfit was only $1.5 \%$.

Since the dislocation density in the crystal was low at the start of the transformation, it was highly unlikely that the misfit dislocations would be supplied from the matrix. For this reason, mechanism 2 was dis carded.

Mechansim 3 requires the nucleation of a dislocation loop inside the prenipitate. No defects, such as stacking faults, were found in the $(\mathrm{Co}, \mathrm{Fe}) 0$ precipitates when they were fully coherent, and it is though that the nucleation of a loop in the perfect precipitate has a rather high energy barrier.

The present observations indicated that the mechanism involving collapse of lhe point defects at the precipitate surface was the one by 
which the precipitate lost coherency. This was highly likely, in view of the observation of the micropores at the matrix-precipitate interface. As was suggested earlier, the transformation-produced . oxygen vacancies would acuumulate near the interface, finally collapsing into vacancy type loops. The loops could expand by climb along the precipitate surface, thus reducily the interstitint wyse atrece field that surrounded the coherent particle. Once a loop was nucleated, the creation of more interface dislocations would follow in a more or less catastrophic way, 3s was suggested by Weatherly and Nicholson. 56 Occasionally, a dislocation might be produced, by plastic deformation of the precipitate surroundings, as was suggested by the observation of the few dislocations that invariably seems to accompany the semi-coherent precipitates.

In summary:

(a). The present usservations on robalt ferrites heated in air, did not support the phase diagram Juggestad by Aukrust and Muan, they were in agreement with the phase diagram reported by Robin. ${ }^{7}$

(b): Annealing in air of cobalt ferrites with a Co/Fc ratio of 0.76 caused the precipitation of $(\mathrm{Co}, \mathrm{Fe}) 0$. These precipitates were initially coherent, and appeared as small square prisms. They lost coherency when they had reached a size between 1200 and $15000^{\circ}$.

(c). Moiré fringes, dislocations and micropores were observed at the matrix-precipitate interfaces.

(d). The misfit dislocations were shown to have a Burger vector of $1 / 2 a_{\text {matrix }}\langle 110\rangle$, and they did not appear to be dissociated. 
(e). The formation of micorpores at the precipitate interfaces was direct evidence of the participation of oxygen vacancies in the transformation process. The pores also migrated along the moving interface. Their shape indicated that they were filled with oxygen.

(f). A mechanism for the pore formation was proposed. It involved the rejection of oxygen vacancies by the cobalt-wustite phase.

(g) The mechanism of loss of coherency was discussed. Loss of coherency of the precipitates was probably initiated by the nucleation of vacancy loops due to oxygen vacancy clustering at the interface: Ocassionally, some dislocations would be produced, possibly by plastic deformation of the matrix spinel. 
V. FINAL DISCUSSION AND REMARKS

The same approach that is now standard in metallurgical investigations has been followed in the study of defects and phase transformations of a ceramic oxide. The success of the entire work depended greatly on the availability of the proper research facilities: the $650 \mathrm{kV}$ transmissiun electron microscope. While much of the general knowledge that has been obtained about defects and phase transformations in metallic systems can be straightforwardly applied to ceramic oxides, some significant differences should be borne in mind. Most metal oxides are strongly ionic due to the large electronegativity of oxygen, and as a consequence, charge neutrality plays a very important role, The creation of vacancies or interstitials in the cation sublattice of the spinel oxide, at'tects the anion sublattice as well (and conversely) making the point defect chemistry quite complicated. Coulomb bonding between ions is also of a much longer range than short range metallic bonding, and this is probably why even relatively small deviations from the stochiometric cation/anion ratio will have a drastic effect. Evidence of this is found more widely as methods of investigation improve, and non-stochiometric oxide crystal that some timc ago were thought to be single phase, sontiain on closer. examination extended structural defects that accomodate the cation or anion deficiencies. Prime examples are found in the oxides of transition metals of the titanium, vanadium and chromium groups. ${ }^{61}$. Not always is the accomodation of non-stochiometry as spectacular as in these shear type oxides, and in the case of cobalt ferrite an imposed increase in cation to anion ratio is simply accomodated by the precipitation of a phase 
with a higher cation to anion ratio: cobalt-wústite. The strong, long range interactions between the ions on different sublattices, makes that phase transformations in oxides can be strongly influenced by the prevailing oxygen partial pressure. Most phase transformations involving a change in the metal/oxygen ratio must then proceed in a layer like fashion from the free surface of the crystal. Whether or not these layers exhibit discrete interfaces will depend on the nature of the transformation. A distinct interface is most likely to develop when the oxygen partial pressure is such that the matrix completely transforms into a new phase, and when the transformation product has a different lattice symmetry than the matrix. Typical examples of layer growth can be observed during the high oxygen partial pressure oxidation of metals. The layering effect poses so many manufacturing problems that properties of practical oxide ceramics are normally controlled by variation of grain size and porosity or by addition of inert inclusions. Also, transformation strains are not easily accomodated, since most ceramic oxides remain intrinsically brittle up to high temperatures, so that precipitates often nucleate cracks. In this thesis it has been shown that a second phese can be precipitated in cobalt ferrite, without destruction of the matrix by transformation strains. The practical relevance of such transformations, however, must be confined to thin film applications. This is not really a great drawback, since thin films are widely used in microelectronics, and two phase oxides films produced by controlled oxidation or reduction, may be of great use. Precipitation can then be added as an extra parameter in optimizing the performance of such thin film devices. 
One of the technologically interesting properties of cobalt ferrites (and many other iron spinels) is that they are ferrimagnetic. Magnetic properties are largely controlled by the mobility of the domain walls, just as mechanical properties are determined by the mobility of dislocations. The motion of the domain walls can be impeded by two basically different interactions: interactions with internal strain tields, or interaction with non-magnetic particles. When a second phase is present in the form of finely dispersed coherent particles, the interaction of the strain fields of the particles with the magneto-striction that accompanies magnetic ordering may be sufficient so as to affect the domain wall mobility very significantly. This second phase may be magnetic also. An undesirable effect is that the magnetization vectors will be deviated by the presence of the internal strain fields, ${ }^{\mathrm{G}_{2}}$ thus leading to a decrease in the magnetic susceptibility. This effect would not be present if the second phase is completely incoherent, so that internal strain fields are absent. In that case, though the second phase should be non-magnetic or anti-ferrimagnetic. Anti-ferrimagnetic particles are of special interest since they can very effectively pin the neighboring ferrimagnetic matrix through exchange anisotropy: 63 A decrease in magnetic succeptibility is then to be expected as resulting from the decrease of the volume fraction of the ferrimagnetic material, from the presence of demagnetization fields, or from closure domains around large precipitates. Dijkstra and West $^{64}$ found experimentally that particles of a size equal to the thickness of the domain wall were most effective. This is in agreement with a simple analysis. ${ }^{4}$ For a 180 degree wall in cobalt ferrite, this would be about $250 \AA$; for a $90^{\circ}$ wall this would be 
about half. An even distribution of particles of this size is aifficult to achieve, unless the decomposition is of a particular nature. In general a spinodal transformation, or large supersaturations are required to produce a high density of nuclei of the second phase. Examination of the phase diagram in air, Fig. 1l, shows that there is a possibility of spinodal decomposition. A spinodally decomposed material seems to be the ideal for a device in which magnetic switching is to occur by coherent spin rotation. The magnetic properties of fine grained polycrystalline covalt ferrites that have been spinodally decomposed have been examined very recently by Takahashi and Fine ${ }^{54}$ and coercive forces of up to $20 \mathrm{k}$ Oe at $77^{\circ} \mathrm{K}$ were reported. Unfortunately, it was not possivle to obtain single crystals in the composition range required for a spinodal decomposition in air. In the present work; at reduced oxygen partial pressures, very high densities $\left(3 \times 10^{17}\right.$ per $\left.\mathrm{cm}^{3}\right)$ of second phase particles were produced by a homogeneous transformation, and it is quite likely that two phase films with such a microstructure would have magnetic properties that are highly desirable. Confirmation has to come from actual magnetic measurements on one micron thick foils of cobalt ferrite that have been heated for 3 to 5 minutes in a reducing $\mathrm{H}_{2} \mathrm{O} / \mathrm{H}_{2}$ atmosphere. 


\section{ACKNOWLEDGEMENTS}

The author is deeply indebted to Professor Gareth Thomas, for continuous guidance and stimulating discussions during the course of this investigation. He also wishes to acknowledge the assistance of $\mathrm{Dr}$. W. Bell in the dislocation contrast examination; $\mathrm{Dr}$. P. Okamoto for numerous discussions cancerning phase transformations; G. Stone for supplying the structure facture computer program;' Dr. D. Olander for kindly providing the partial pressure gage; R. Clem for performing the chemical analysis; D. Jurica for attending to the well being of the high voltage electron microscope at all times of day; his colleages for stimulating discussions and enthusiasm; Dr. E. Nes for critical reading of some sections; and finally the United states Atomic Energy Commission for financial support through the Inorganic Materials Research Division of the Lawrence Radiation Laboratory. 


\section{REFERENCES}

1. R. W. G. Wijckoff, Crystal Structures, Interscience Pub. Inc., N.Y., 1951.

2. E. W. Gorter, Philips Res. Rep. 2, 295 (1954).

3. E. C. Snelling, Proc. British Ceram. Soc. 2, 151 (1964).

4. A. Broese van Groenou, P. F. Bongers, and A. L. Stuyts, Mater. Sci. Eng., 3, 317 (1968).

5. W. Muller and H. Schmaltzreid, Ber. Bunsenges. Physik. Chem. 68,270 (1964).

6. E. Aukrust and A. Maun, in Reactivity of Solids, North Holland Publ. Co., 1964.

7. J. Robin, Ann. Chim. 10, 393 (19555).

8. W. L. Roth, J. Phys. Chem. Solids 25, 1 (19.64).

9. T. Nagamiya, K. Yosida and R. Kudo, Adv. Phys. 4, I (1955).

10. S. Chikazumi, Physics of Magnetism, John Wiley and Sons, Inc., N.Y. 1964, p. 98.

11. K. Hoselitz, Proc. British Ceram. Soc. 2, 97 (1964).

12. H. Schmaltzreid, Progress in Solid State Chemistry 2, Pergamon Press, 1965:

13. H. Schmaltzreid and J. D. Tretjakow, Ber. Bunsenges. Physik. Chem. 70, $180(1966)$.

14. G. Valensi, La Metal. Ital. $\underline{3}, 77$ (1950).

15. V. I. Arkharov and K. M. Yaerski, Zh. tekh. fiix. SSSR 14, 132 (1944).

16. E. A. Gulbransen and K. F. Andrew, J. Electrochem. Soc. 98, 241 (195i).

1\%. A. Bergstein, Màt. Res. Bull. 3, 787 (1960),

18. C. Wagner and K. Grunewald, Z. Phys. Chem. B40, 455 (1938).

19. M. H. Lewis, Phil. Mag. 13, 777 (1966). 
20. M. J.Makin, Phil. Mag. 18, 637. (1968).

21. M. F. Ashby and L.M. Brown, Phil. Mag. 8, 1083 (1963).

22. M. Rühle, M. Wilkins and U. Essmann, Phys. Stat. Solidi 11, 819 (1965).

23. G. Thomas and W. L. Bell, in Lattice Defects and Their Interactions, ed. R. Hashiguti, Gordon \& Breach, N. Y. 1967, p. 477.

24. W. L: Bell and G. Thomas, Proc.:27th Annual FMSA Meeting, Claitor's Publ., 1969, p. 156.

2j. L. M. Howe, J. F. McGurn and R: W. Gilbert, Acta Met. 14, 801 (1966).

26. K. P. Chik, Phys. Stat. Solidi 16, 685 (1966).

27. J. Hornstra, Proc. 4th Inț. Symp. Reactivity of Solids, 563 (1961).

28. M. L. Kronberg; Acta Met. 2, 597 (1957).

29. M. H. Lewis, Phil. Mag. 17, 48i (1968).

30. P. B. Hirsch, A. Howie, R. R. Nirholgor, D. W. Tashley, and M. J."Whelan, Electron Microscopy of Thin Crystals, Butterworths, London, 1965.

31. S. Katagiri, M. Nishigaki, and H. Yokodoro, J. Electron. Microsc. $\underline{3}, 215$ (1968).

32. N. H. Nachtrieb, Spectrochemical Analysis, McGraw-Hill, New York, 1950.

33. W. H. Stobbs, private communication.

34. D. J. H. Cockayne, I. L. F. Ray and M. J. Whelan, Phil. Mag. 20, 1265 ( 1969$)$.

35. M. H. Lew1s, Phil. Mag: 14, 1003 (1966).

36. H. Blank and S. Amelinckx, J. Appl. Phys. 34, 2200 (1963).

37. J. Washburn, G. N. Groves, A. Kelly and G. K. Williamson, Phil. Mag. 2. $991(1960)$. 
38. P. Delavignette and S. Amelinckx, Appl. Phys. Letters $\underline{2}, 236$ (1963).

39. C. A. May and K. H. G. Ashbee, Phil. Mag. 18, 61 (1968).

40. J. Hornstra, Mat. Sci. Res: 1, 88 (1963).

41. R. M. Bozorth, E. F. Tilden and A. J. Williams, Phys. Rev. 99, 1788 (1955).

42. G. Remaut, A. Lagasse, and S. Amelinckx, Phys. Stat. Solidi, I, 447 $(1964)$.

43. C. Kittel, Introduction to Solid State Physics, J. Wiley \& Sons, Inc. N. Y., 1966.

44. R. Gevers, P. DeJavignette, H. Blank and S. Amelinckx, Phys. Stat. Solidi $\underline{4}, 383(1964)$.

45. R. S. Tebble, Magnetic Domains, Methuen \& Co. LTD, London, 1969, p. 40.

46. E. Nes, Ph. D. Thesis, U. C. Berkeley, UCRL-19608, August 1970.

47. J. B. Newkirk and W. G. Martin, Trans. AIME 212, 398 (1958).

48. M. Hansen, Constitution of Binary Alloys, McGraw-Hill, N.Y., 1958, p. 472 .

49. Gordon Research Conference, Aug. 24-28, Tilton, N. H., 1970 (unpublished).

50. J. W. Christian, Transformations in Metals and Alloys, Pergemon Press, 1965.

51. M. E. Fine, The Chemistry of Extended Defects in Non-metallic Solids, Ed. L. Eyring and M, O'Keeffe, North-Holland Publ. Co., Amsterdam, 1970, p. 575.

52. G. P. Wirtz and M. E. Fine, J. Am. Ceram. Soc. 51, 402 (1968).

53. A. J. Ardell and R. B. Nicholson, Acta Met. 14, 1295 (1966), and J. Phys. Chem. Solids 27, 1793 (1966). 
54. M. Takahashi and M. Fine, Am. Ceram. Soc., West Coast Annual Regional Meeting, October 1970.

55. L. L. Sass, T. Mura and J. B. Cohen, Phil. Mag. 16, 679 (1967).

56. G. C. Weatherly and R. B. Nicholson, Phil. Mag. 17, 801 (1968).

57. R. Gevers, Phil. Mag. I, 1681 (1962).

58. R. S. Barnes and D. J. Mazey, Proc. Roy. Soc. A, 275, 47 (1963).

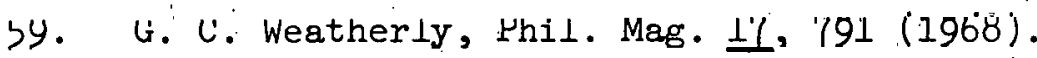

60. A. Eikum and G. Thomas, Acta Met, 12, 537 (1.964).

61. A. Magnëli, The Chemistry of Extended Defects in Non-metallic Solids, ed. L. Eyring and M. O'Keeffe, North-Holland Puh, Co, Amsterram, 1970.

62. Ya. S. Shur, A. A. Glazer, Yu. N. Dragoshanskiy, V. A. Zaykova and G. 3. Kandaurova, Izd. Akad. Nauk. SSSR 28, 553 (1964).

63. W. L. Roth, The Chemietry of Extended Defects in Non-Metallic Solids, ed. I. Eyring and M. O'Keeffe, North-Holland Pub. Co., Amsterdam, $1970 ;$ p. 455.

64. L. J. Dijkstra, and C. West, Phys. Rev. 79, 974 (1950). 


\section{FIGURE CAPTIONS}

Fig. 1a. Phase diagram at 1.atm. of air. 6

Fig. Ib. Phase diagram at 1 atm of air.?

Fig. 2. Schematic octahedral cation distribution. 5

Fig. 3. Calibration curves for the hot stage.

Fig. 4. Partial pressure gage readout. The partial pressures of the most important gases in the electron microscope are listed.

Fig. 5. Defect distribution around a nearly perfect conical etch pit. The projected defect density is uniform.". The foil is about one micron thick at the outer thickness fringes.

Fig. 6. Bright and dark field pair of defects.

Fig. 7. Defects imaged with two different operating vectors. A loop is marked $\mathrm{L}$.

Fig. 8. Charge of projected defect density with time of observation. The spherical clusters do not change to loops, and conversely.

Fig. 9. Observed projected defect density versus time.

Fig. 10. Defects formed during electron microscope observation at liquid nitrogen temperatures. The contrast indicates that now small tetrahedra are formed, instead of spherical clusters.

Fig. 11. Dislocation dissociation in spinel, Hornstra. 27

Fig. 12. Dislocation network in cobalt ferrite. This configuration excludes wide fourfold dissociation.

Fig. 13. Dislocations in cobalt ferrite do not seem to be twofold dissociated.

Fig. 14. Comparison between calculated and actual dislocation images. The magniture of the Burgers vector is found from the fringe 
spacings, not from their intensity $\bar{g}_{1} \cdot \bar{b}=2$ for this image.

Fig. 15. Diffraction geometry for a systematic set of reflections.

Fig. 16. Computed and calculated dislocation images with $\vec{g}_{1} \cdot \vec{b}=1$. The splitting of the central image fringe must be due to narrow twofold dissociation. 33

Fig. 17. Dislocation image in high resolution weak beam mode. ${ }^{34}$ At the node, the foil is about $2000 \AA$ thick. High rasolution is only obtained in the thin part of the foil.

Fig. 18. Deflection of clectron beam by Lorentz forces, and consequent change in deviation parameter ' $s '$

Fig. 19. Geometrical construction showing lattice rotation at coherent tetragonal twin boundary. The angle of rotation $\alpha$, is determined by the magnetostriction parameter, $\lambda_{100}$.

Fig. 20.. Specimen in the field of the objective lens. $\bar{H}_{\text {obj }} \cong 7$ kgauss at the specimen.

Fig. 2I All magnetization vectors must have a component nearly equal, and parallel to $\overline{\mathrm{H}}_{\mathrm{obj}}$, if domains are to be observed.

Fig. 22. Overapping ferrimagnetic domain walls. The domain wall plane can be derived from a geometrical analysis of this image.

Fig. 23. Geometrical analysis of Fig. 22.

Fig. 24. Ferrimagnetic domain walls in cobalt ferrite.

Fig. 25. Schematic arrangement of the domain walls, and magnetization vectors in Fig. 24. The change in $\bar{s}$ is indicated at the walls, together with the resultant change in fringe nature.

Fig. 26. In this (III) foil, three different magnetization vectors are present, as was illustrated in Fig. 21. 
Fig. 27. Motion of zigzag walls. A reference point has been marked. Note in $B$, that the pitch of the zigzaging can be as low as 0.25 micron, as is evidenced by the oscillations in the extinction contour.

Fig. 28. Doubling of Kikuchi line evidences a lattice rotation across the wall. This particular configuration is only possible as long as the foil can accommodate the rotation by buckling.

Fig. 29. The $90^{\circ}$ block walls are not necessarily planar. The foil edges are the nucleation sites of new domains.

Fig. 30. Effect of an elastic bending stress on the domain arrangement.

Fig. 31.. Two types of precipitates are observed in a foil of cobalt ferrite, after heating for $3 \mathrm{~min}$ at $500^{\circ} \mathrm{C}$, in the electron microscope.

Fig. 32. Stereo pair of area in Fig. 31. Study of this pair will show that the larger precipitates are on the foil-surfaces, while the very small ones are deeper inside the foil.

Fig. 33. Location of the precipitates in a foil heated for $3 \mathrm{~min}$ at $500^{\circ} \mathrm{C}$, in the hot stage of the electron microscope.

Fig. 34a. Diffraction pattern of area shown in Fig. 31.

Fig. 34b. Solution to Fig. 34a.

Fig. 35\% Resolution of the cobalt-wüstite reflections at higher order reflections.

Fig. 36. Bright and dark field imaging of precipitate reflections, The reflections isolated by the objective aperture are encircled. Note that in $d$, the flat sides of the precipitates are parallel to $(100)$. 
Fig. 37. Dark field images of indicated reflections. The very small precipitates $(\sim 30-40 \AA)$ are $(\mathrm{Co}, \mathrm{Fe}) 0$, the larger ones are a mixture of hexagonal and cubic cobalt. Note that the (Co, $\mathrm{Fe}) 0$ precipitates randomly.

Fig. 38. No preferential decoration of dislocations by the ( $\mathrm{C}, \mathrm{Fe}) \mathrm{O}$ phase is observed. This indicates that the nucleation of this phase is homogeneous.

Fig. 39. Decoration of foll edges, and surface steps by cobalt metal.

Fig. 40. Time sequence showing how the reaction proceeds in a wedge shaped foil. The elapsed time is indicated. Note the shrinking of the inner reaction zone. Note also how the matrix is consumed around the growing cobalt particles, especially at the Poil cdge.

Fig. 41. Strain contrast of the (Co,Fe)o precipitates with three different operating vectors. The contrast indicates that the partirles are spherical and coherent.

Fig. 42. Transforming foil showing the deterioration of the orientation relationship between the metal and the matrix, in the later stages.

Fig. 43. Cobalt grains in late transformation stage show clear substructure.

Fig. 44. Analysis of a cobalt grain shows that the substructure is due to the presence of cubic and hexagonal cobalt.

Fig. 45. Cobalt grain showing nearly periodic twinning or faulting. The substructure acts as a phase grating, giving rise to a succession of very closely spaced diffraction spots. 
Fig. 46. Modulated (Co;Fe)o precipitate arrangements can be found after. transformation, if the transformation to metal + spinel does not occur. The modulation does not originate in spinodal decomposition.

Fig. 47. (Co,Fe)o precipitates as small square prisms, bounded by $\{100\}$ planes, when cobalt ferrite is heated for $10 \mathrm{~min}$ at $1230^{\circ} \mathrm{C}$ in air. Note the particular shape of the line of no contrast when $g=440$.

Fig. 48. Contrast of prism of (Co,Fe)O, when viewed end on. Agreement is formed with theory. 55

Fig. 49. Decoration of dislocation by (Co, Fe)O shows that the transformation is heterogeneous at $1230^{\circ} \mathrm{C}$ in air. Note that there is still evidence of strain.

Fig. 50. Large precipitates of $(\mathrm{Co}, \mathrm{Fe}) \mathrm{O}$ in cobalt ferrite after heating. for $60 \mathrm{~min}$ at $1230^{\circ} \mathrm{C}$ in air. Note the presence of a few dislocations, and the peculiar interface contrast.

Fig. 51. After 20 hours at $1230^{\circ} \mathrm{C}$, the precipitate morphology has changed little. The diffraction pattern of the encircled area identifies the precipitates as ( $\mathrm{Co}, \mathrm{Fe}) \mathrm{O}$.

Fig. 52. Moiré fringes at precipitate interfaces with two different diffracting vectors.

Fig. 53. Large ( $\mathrm{Co}, \mathrm{Fe}) 0$ precipitate at the foil edge. The geometry is drawn in perspective. The foil surfaces intersect the precipitate in the shaded areas. "Bright spots" at the interface are marked 1. Possible interface dislocations are marked 2.

Fig. 54. Tilting experiment showing the presence of interface dislocations. 
Fig. 55. Focusing experiment showing possibility of distinguishing between moiré fringes and dislocations.

Fig. 56. Diffraction experiments showing that the "bright spots" are actually micropores at the matrix-precipitate interface. 


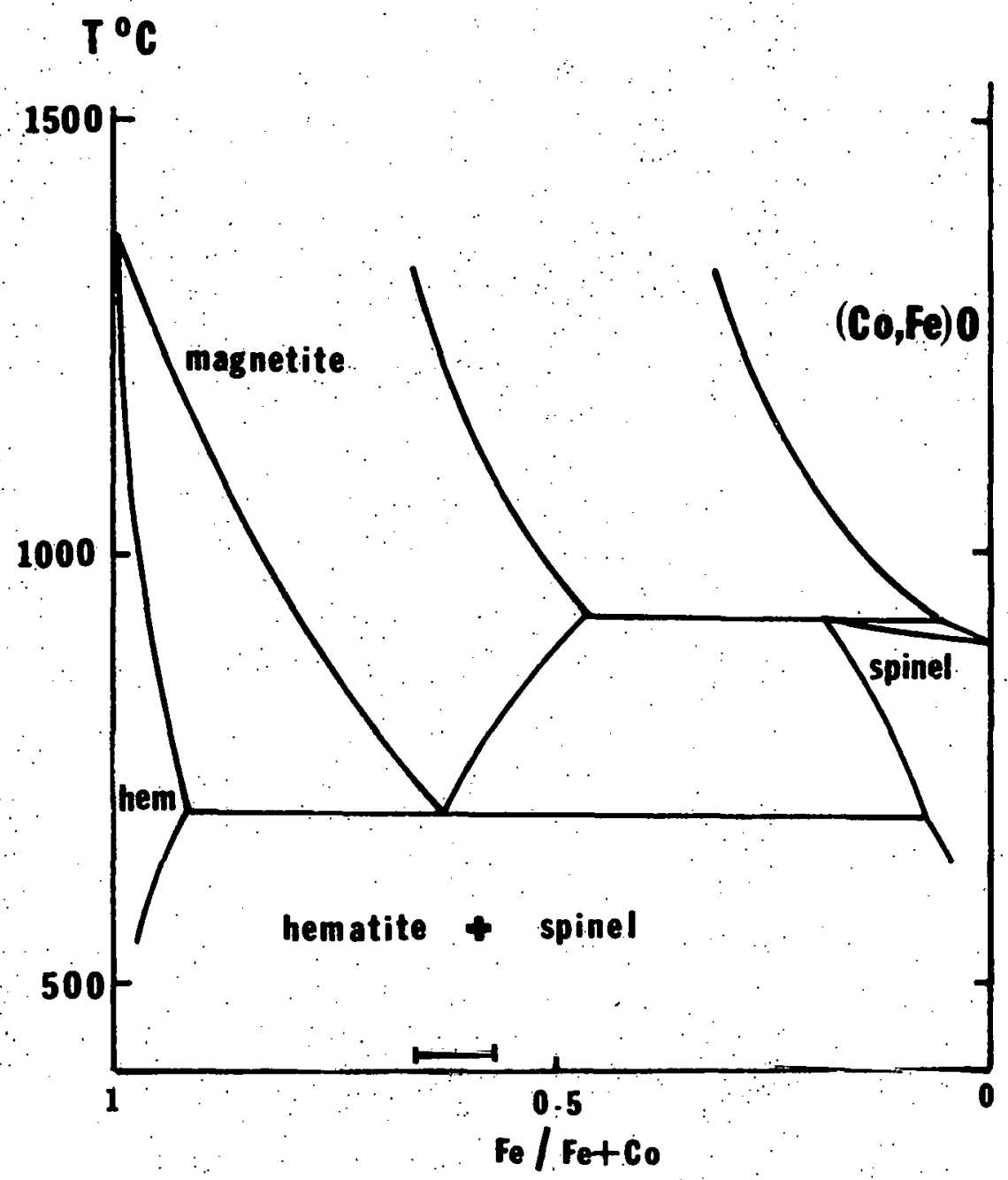

XBL $7010-6693$

Figure la 


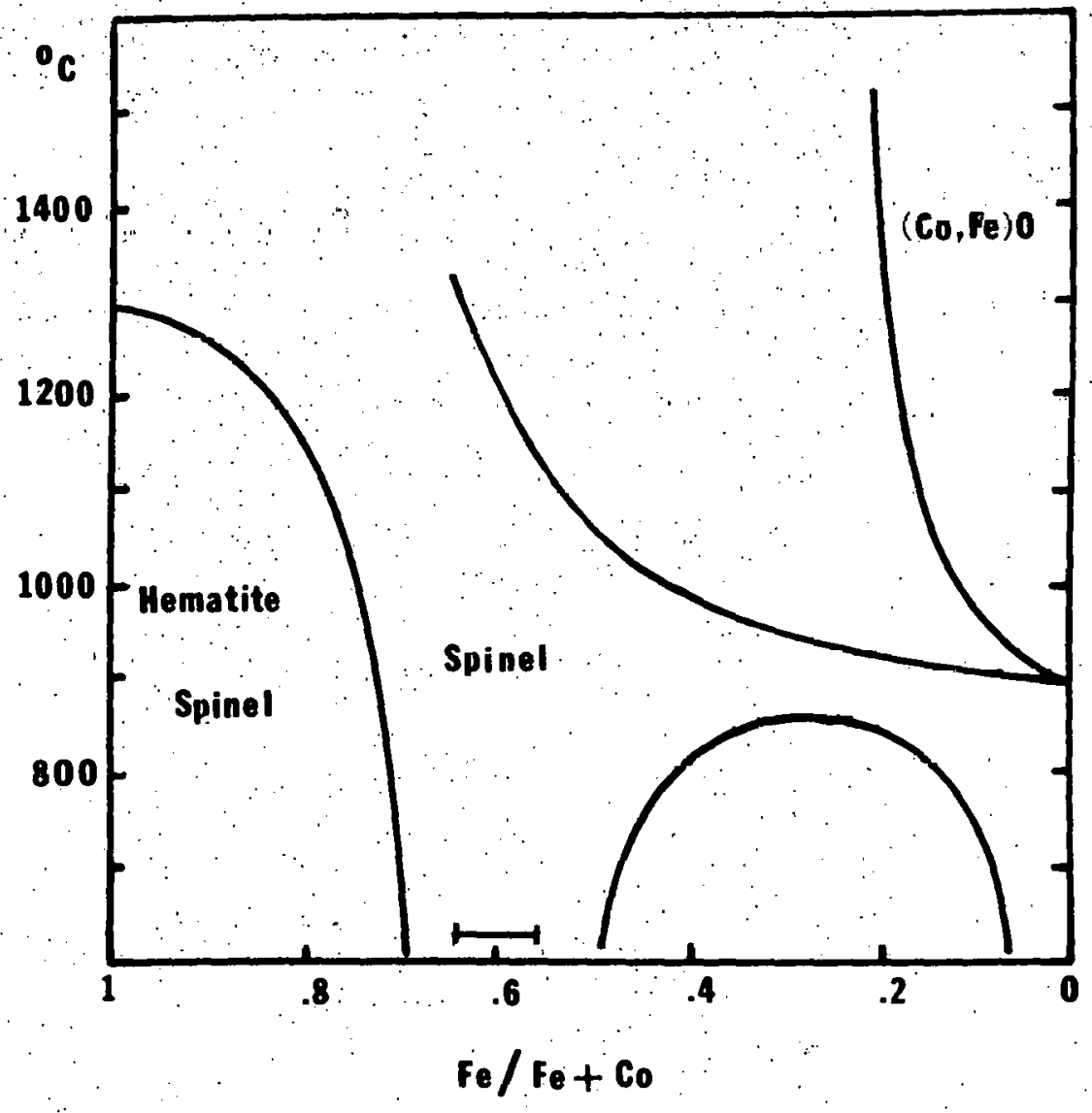

XBL $7010-6692$

Figure $1 \mathrm{~b}$ 


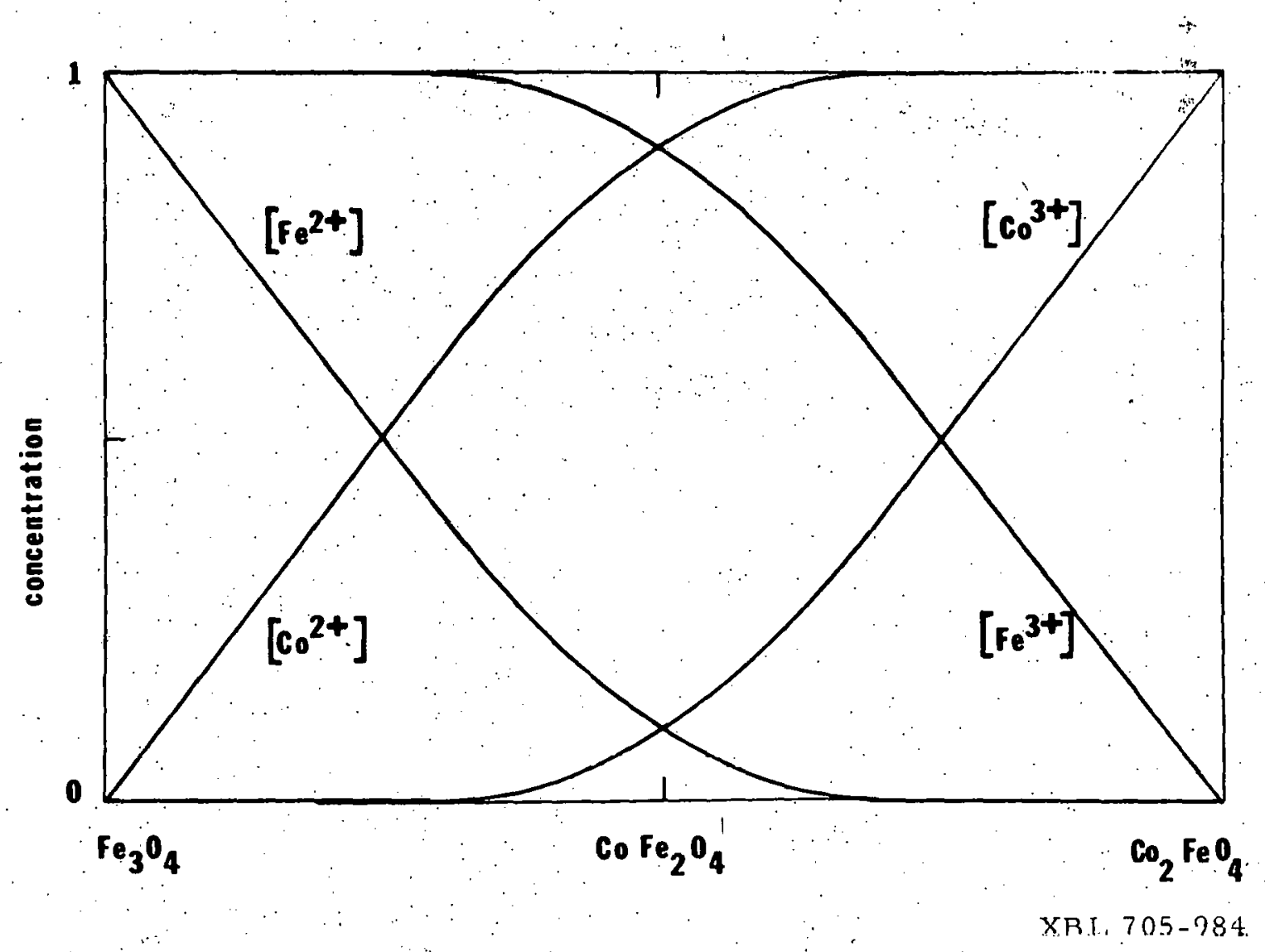

Figure 2 

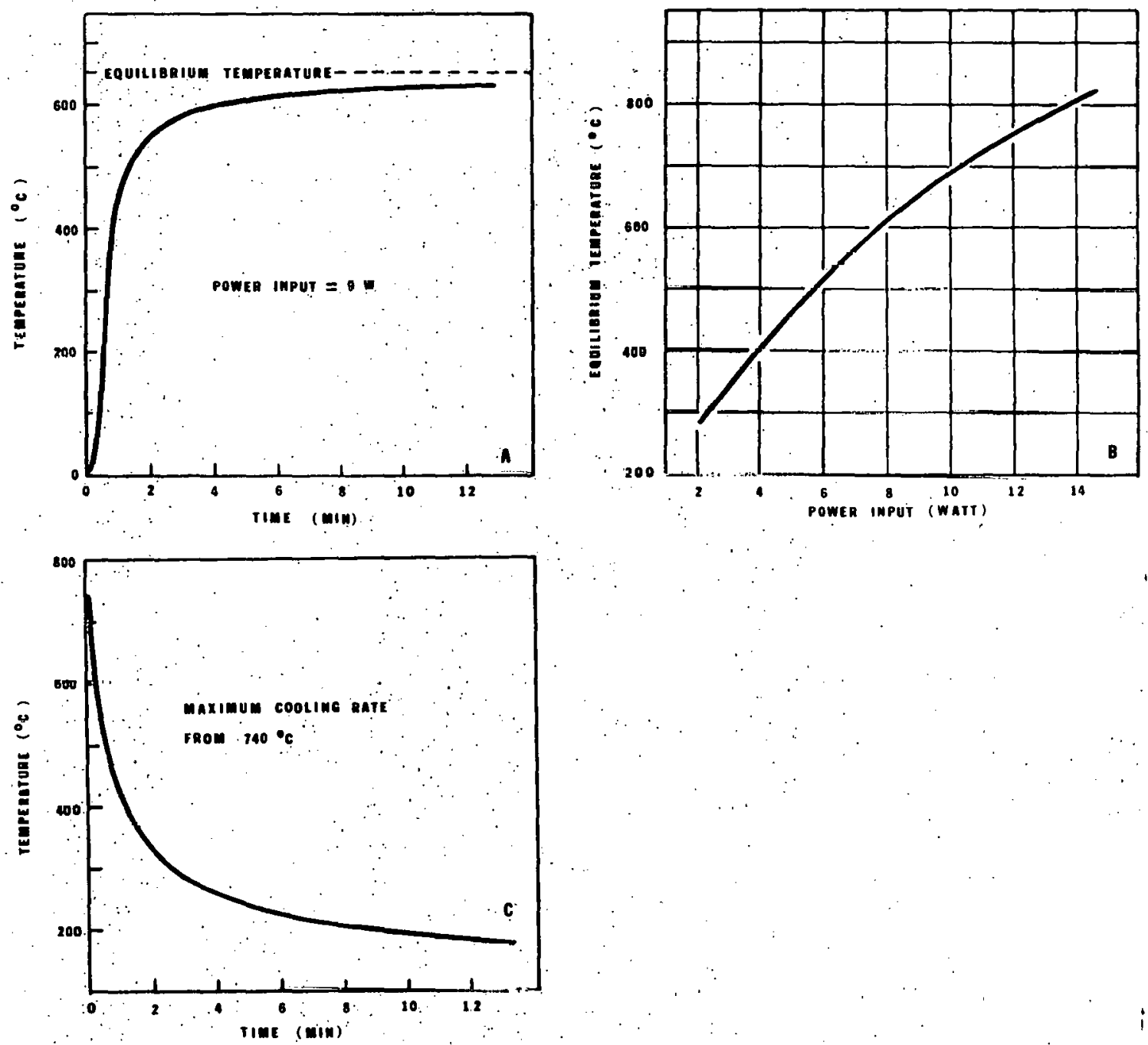

$X B \perp 709-6613$

Figure" 3 


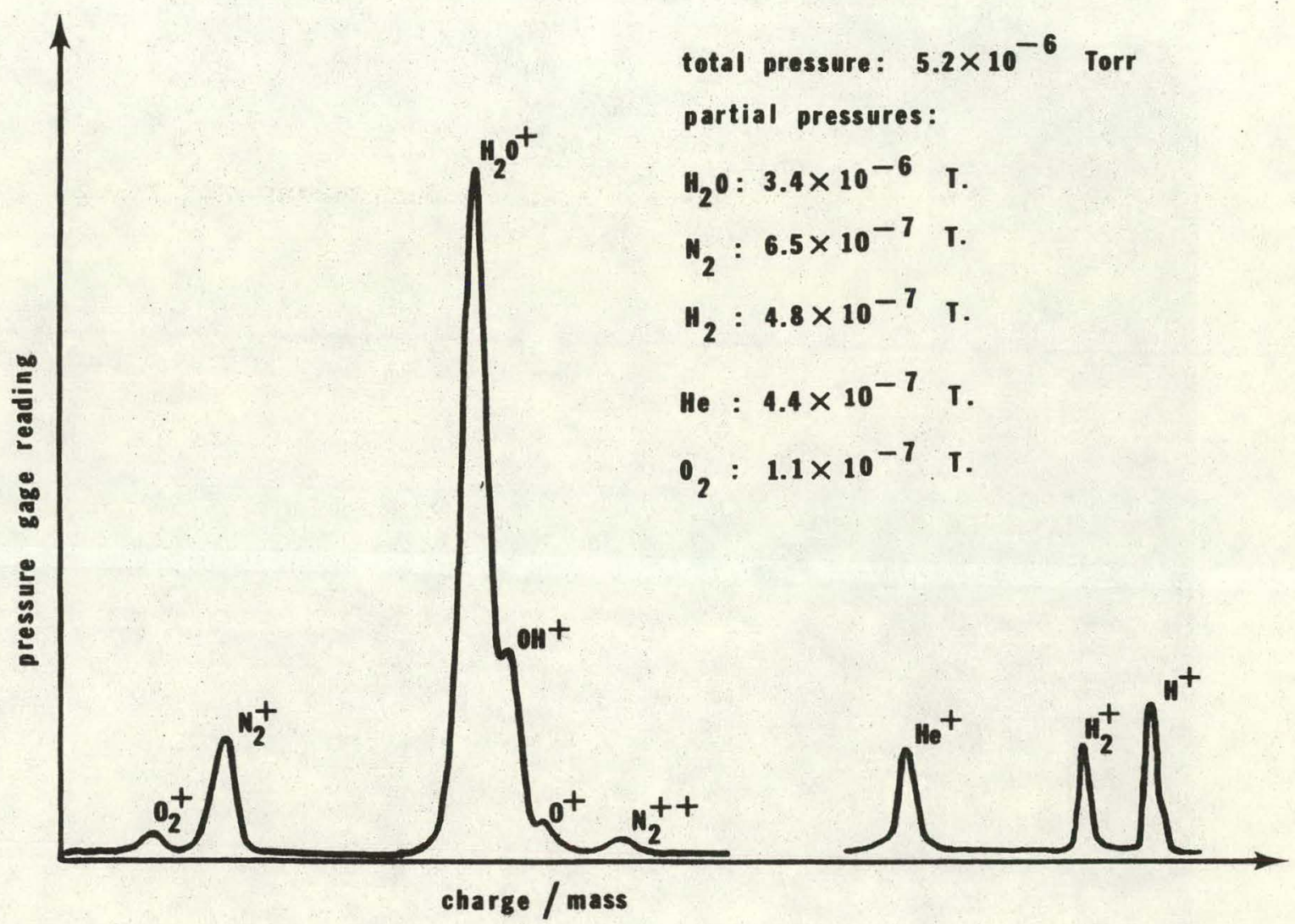

Figure 4 


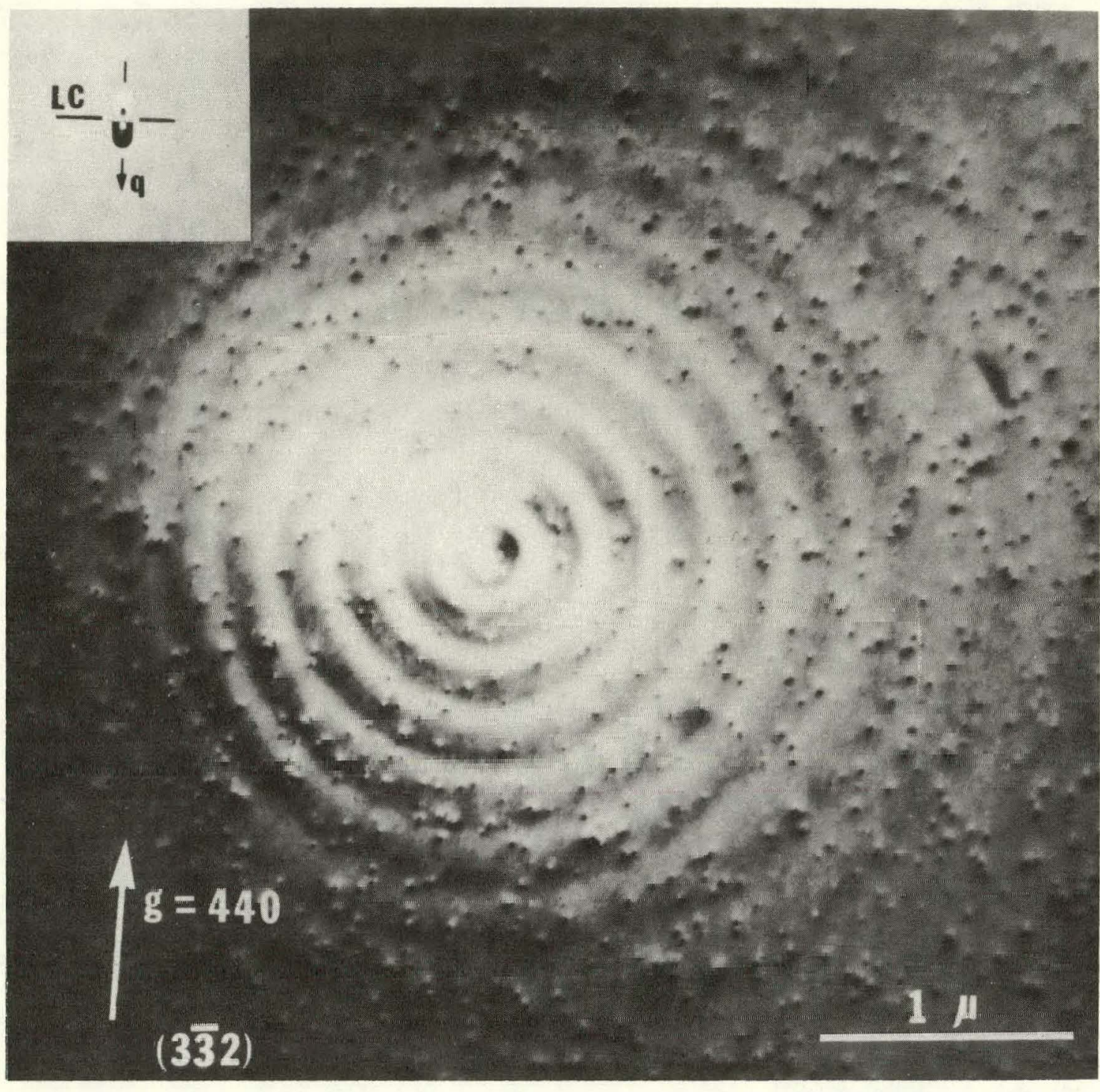

XBB706-2772

Figure 5 

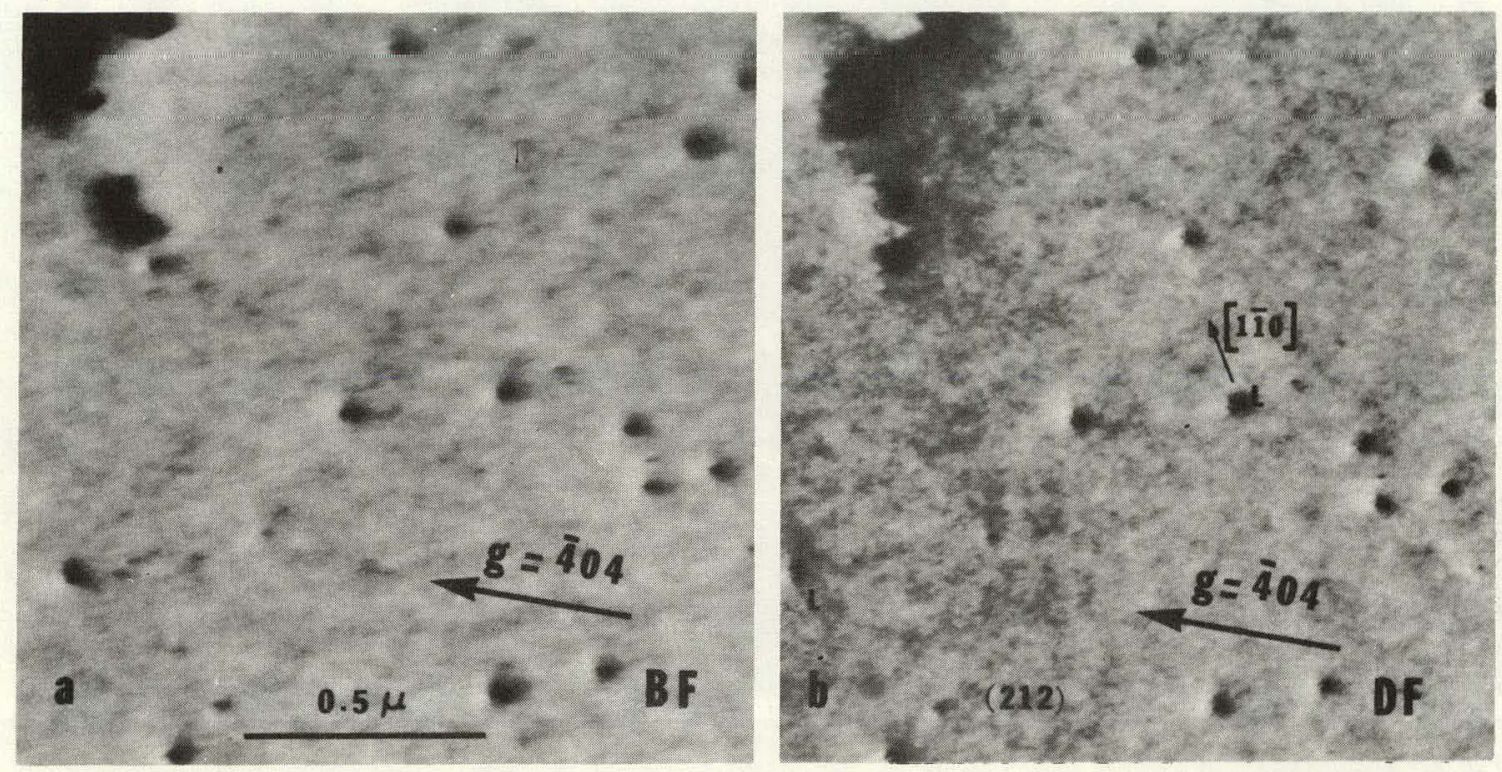

XBB706-2770

Figure 6 

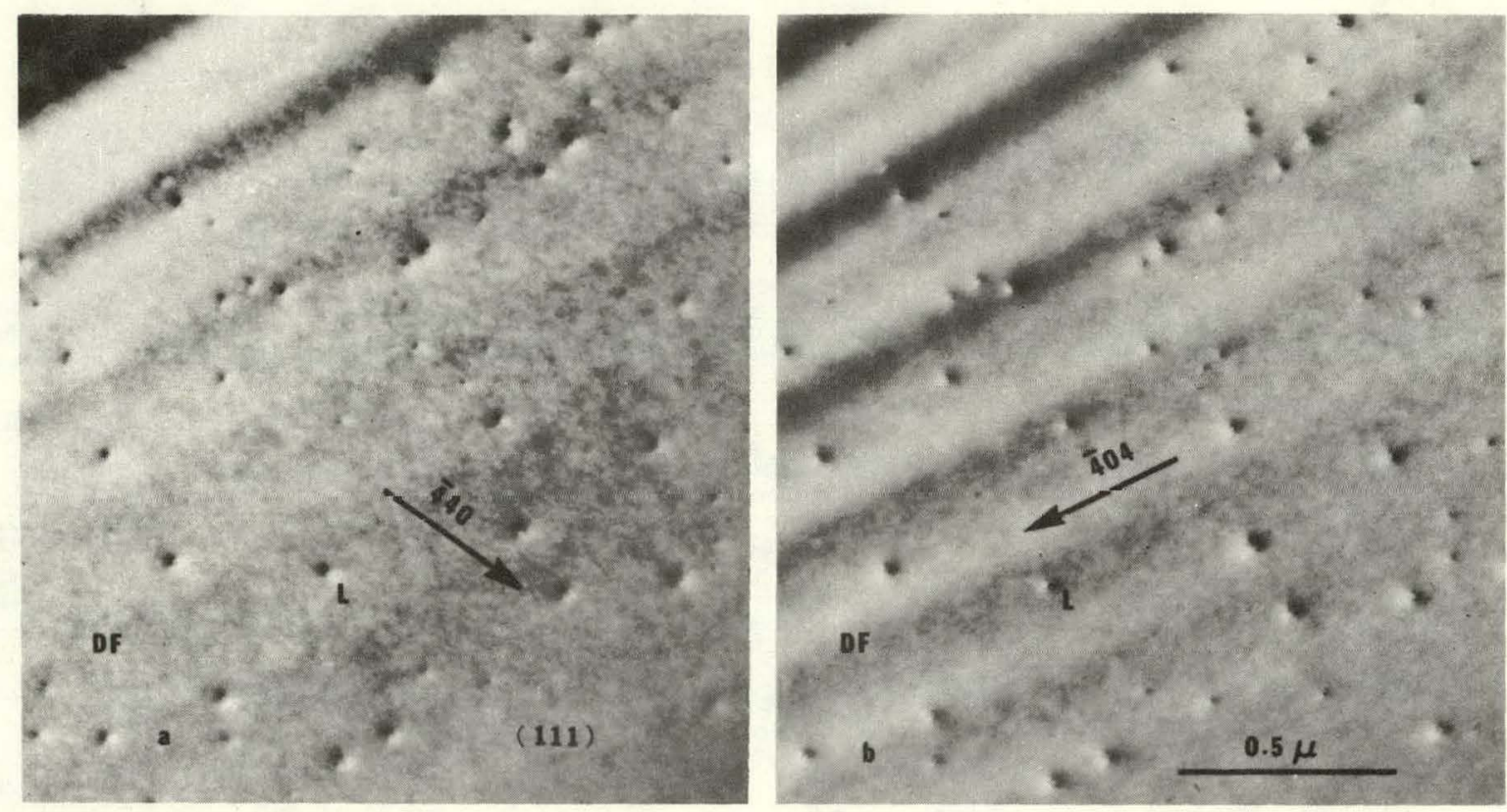

XBB706-2769

Figure 7 

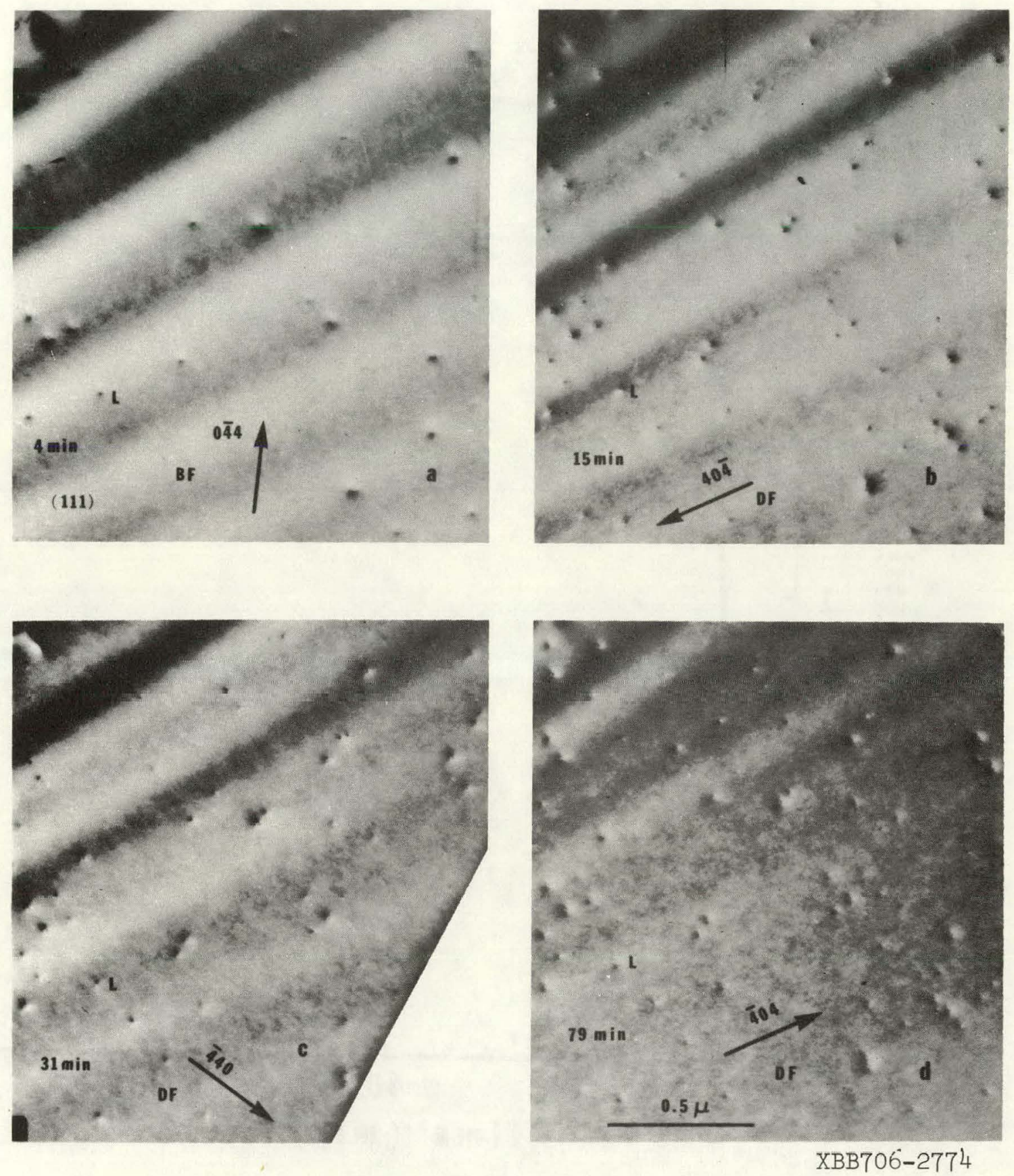

Figure 8 


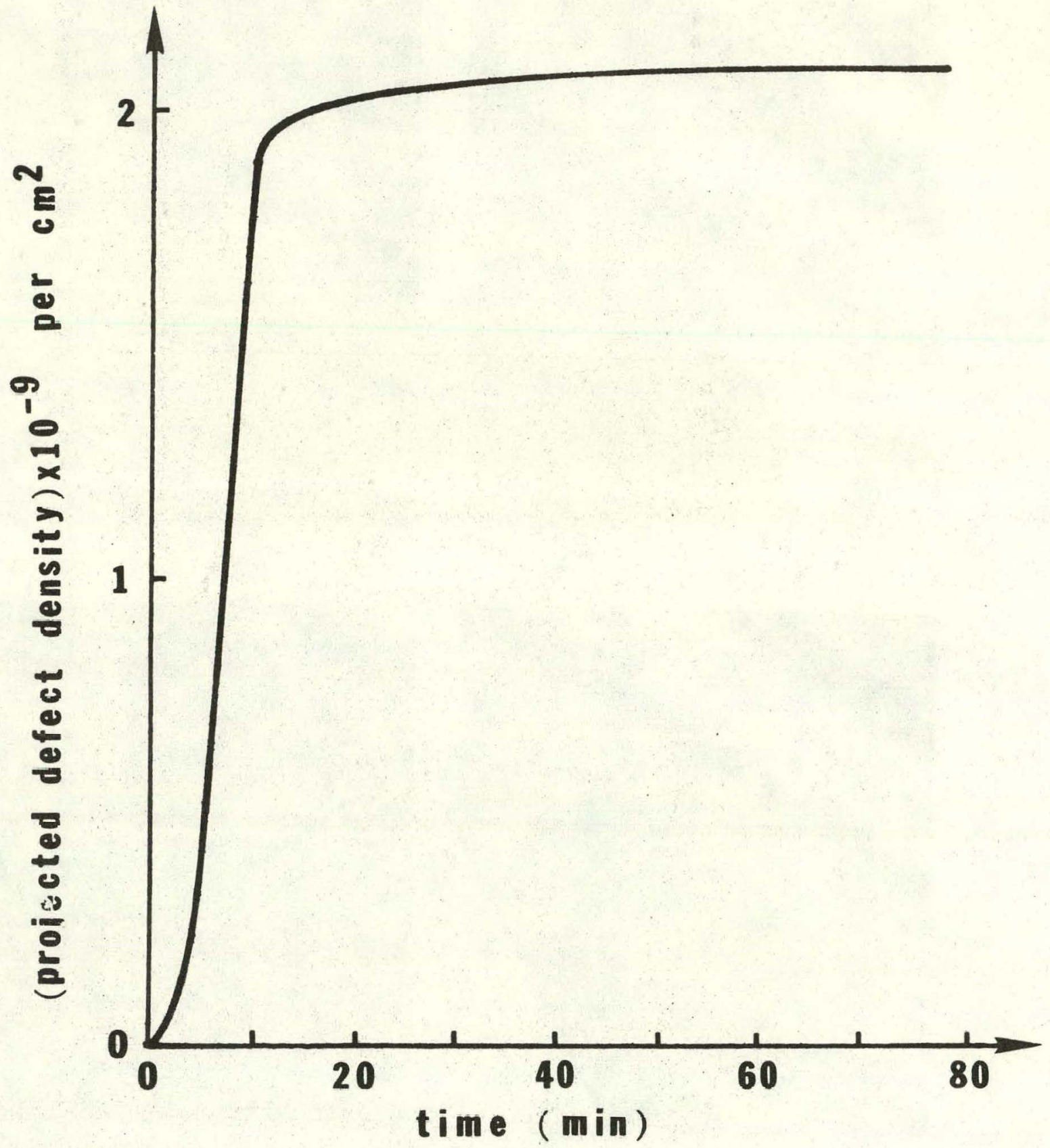

XBL 706-1217

Figure 9 
-87-
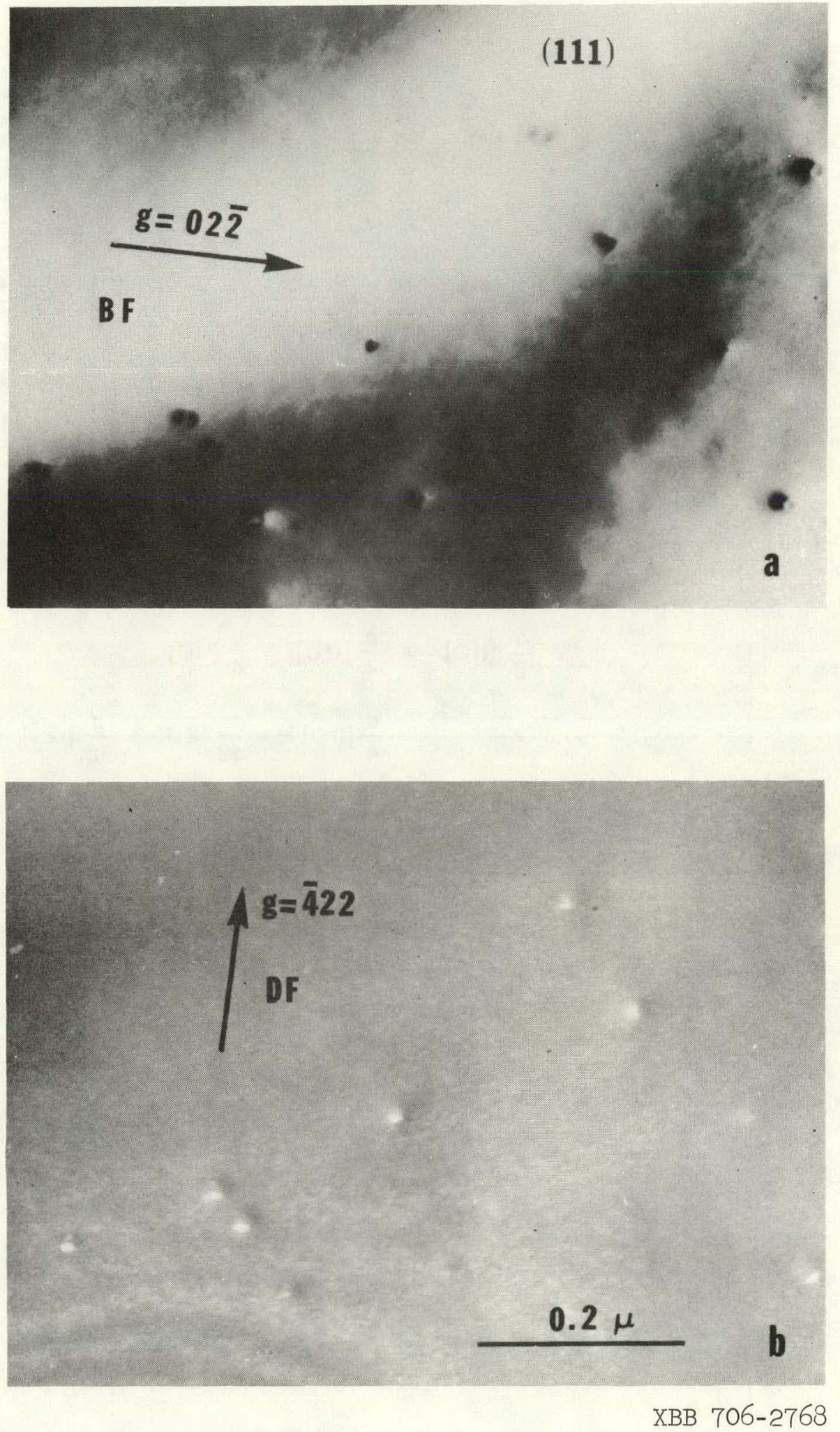

Figure 10 


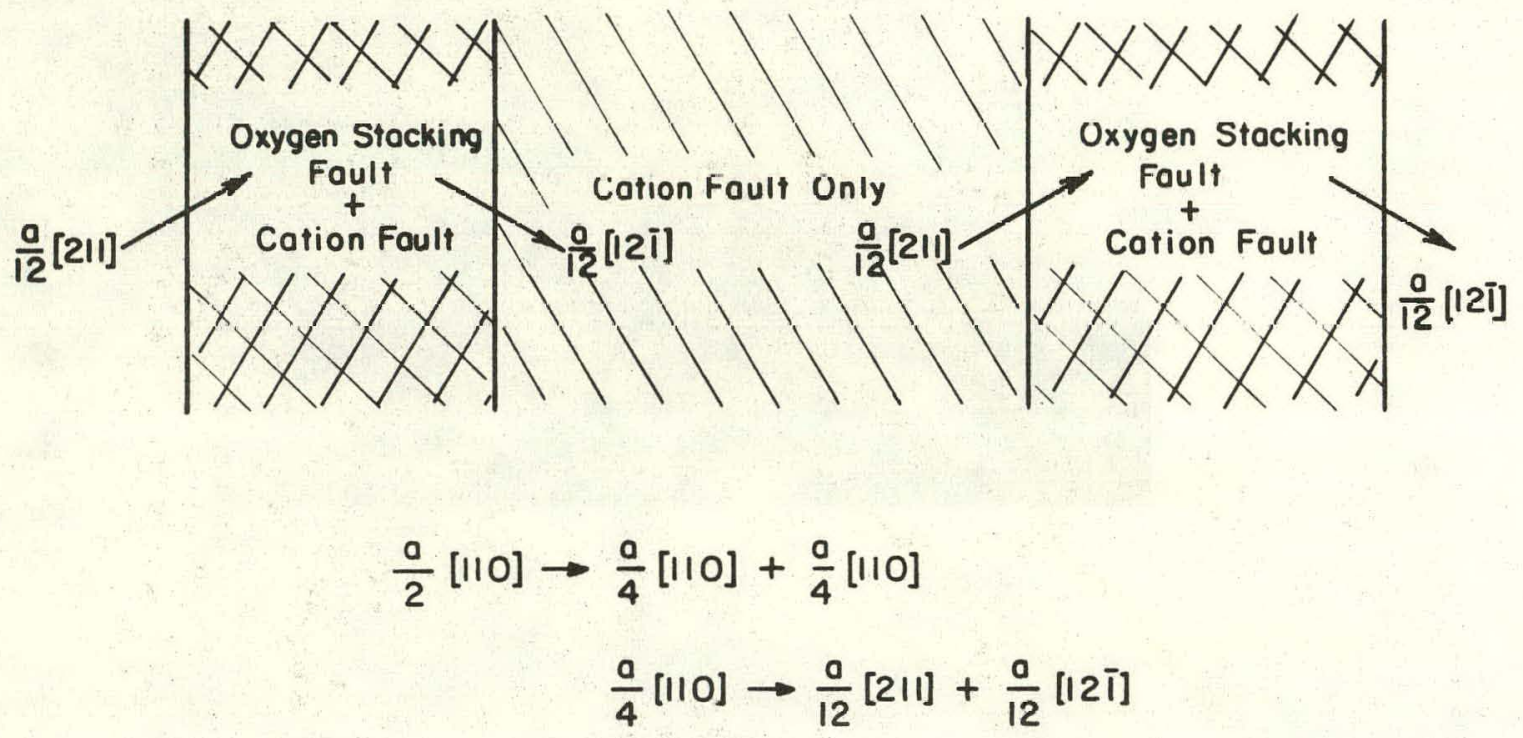

XBL 701-205

Figure 11 


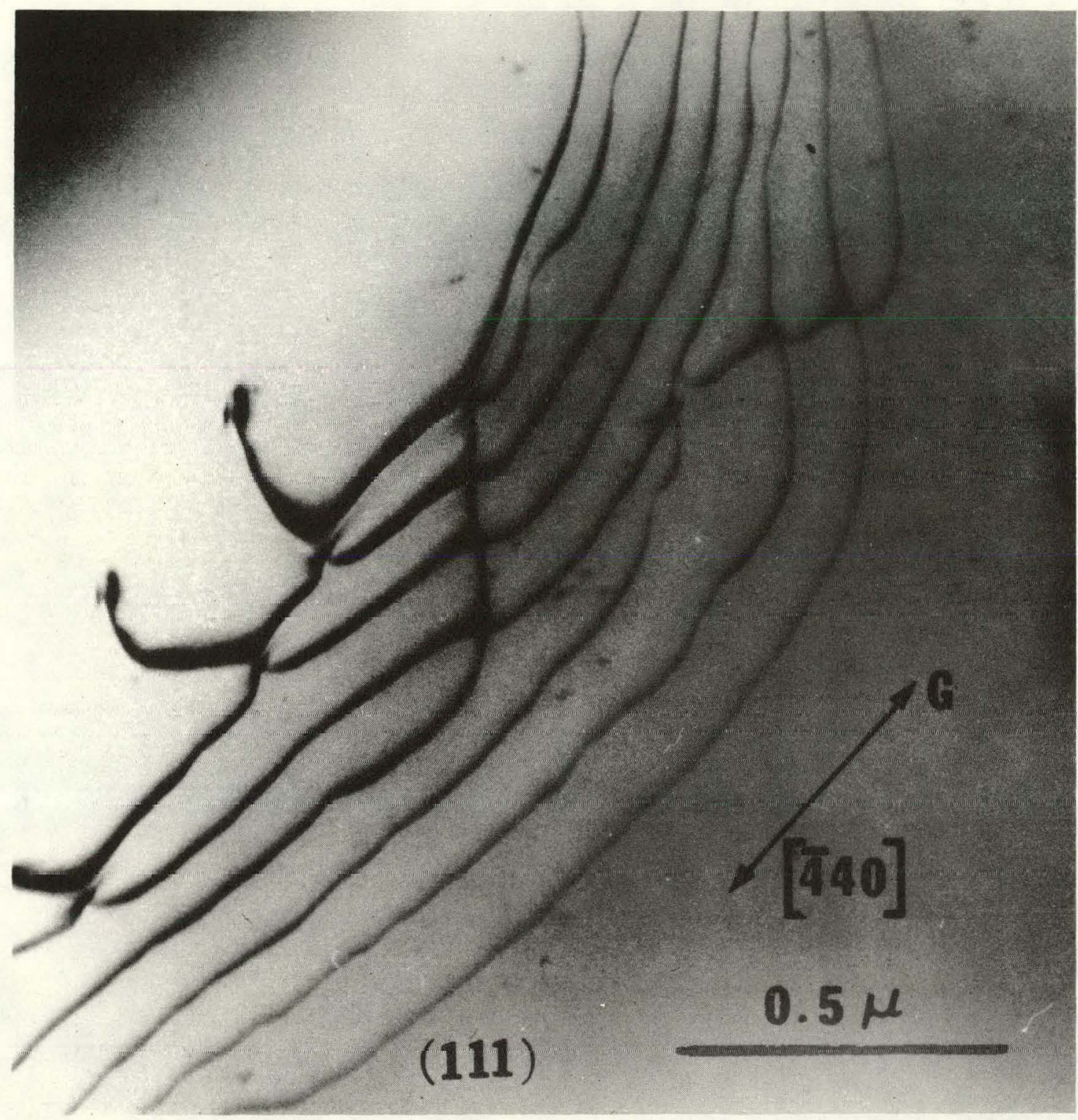

XBB706-2773

Figure 12 

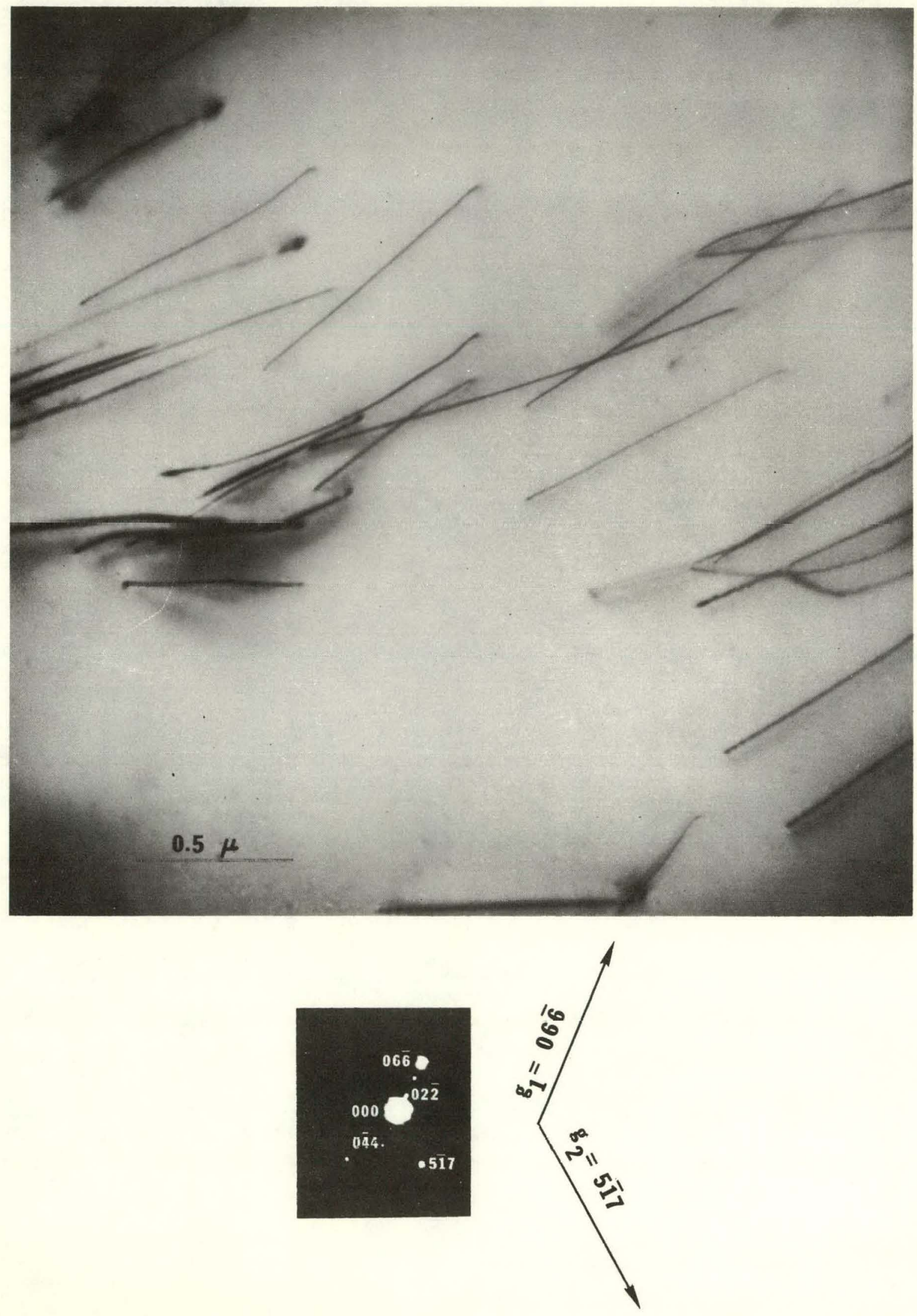

XBB709-4341 


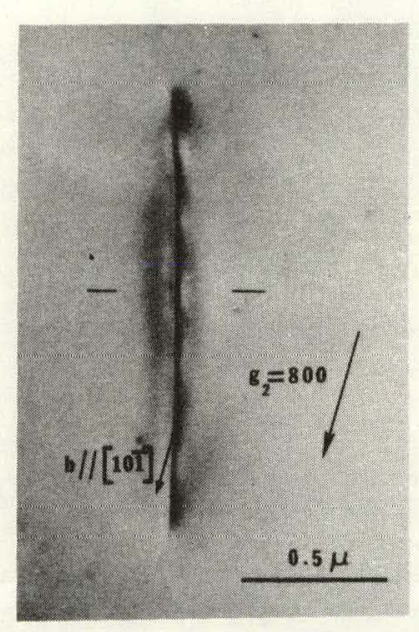

EXPERIMENTAL BRIGUT FIELD

$m_{800}=0$

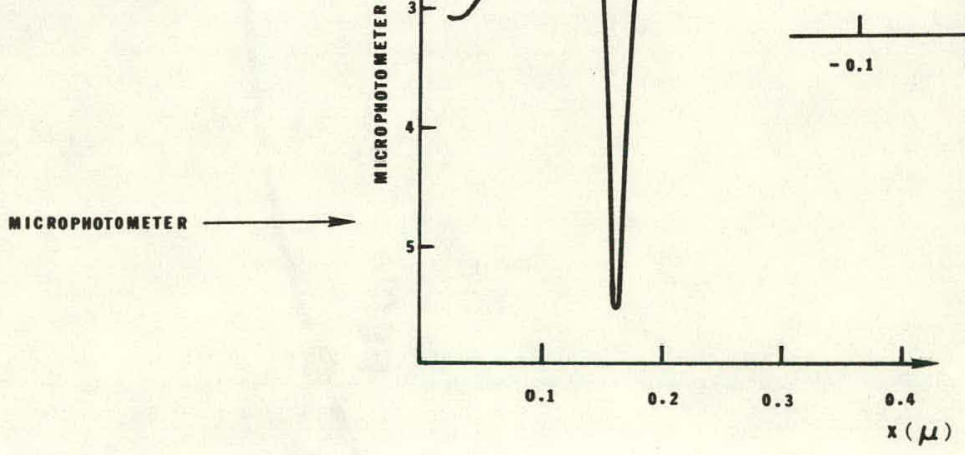

XBB707-3328

Figure 14 


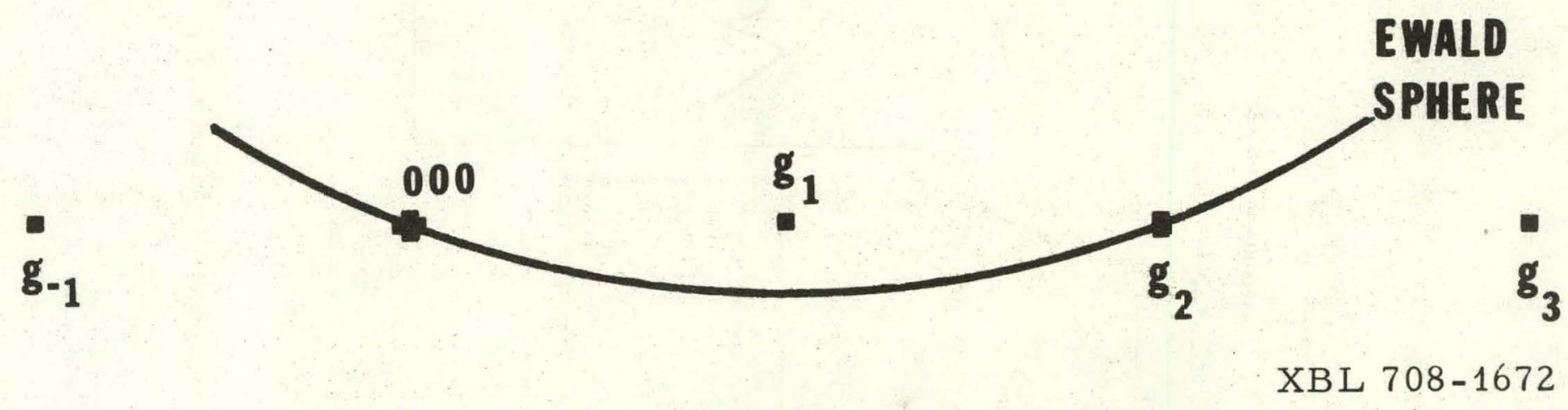

Figure 15 


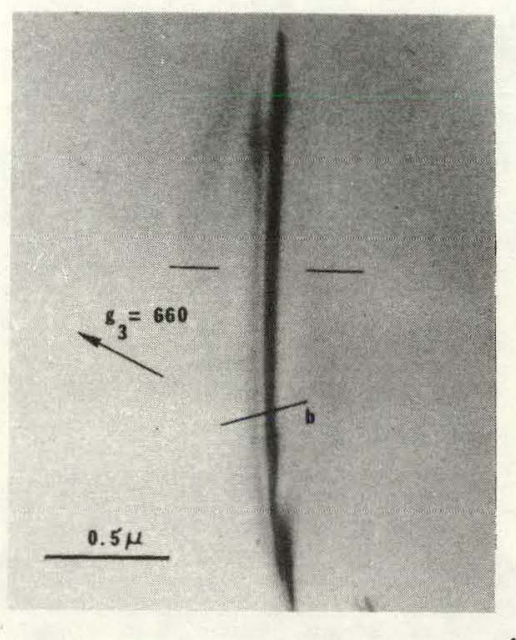

EXPERIMENTAL BRIGUT FIELO

$w_{660}=0$

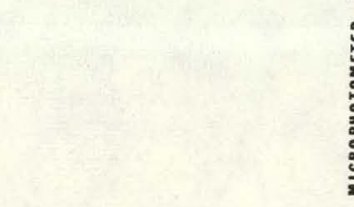

MICROPHOTOMETER
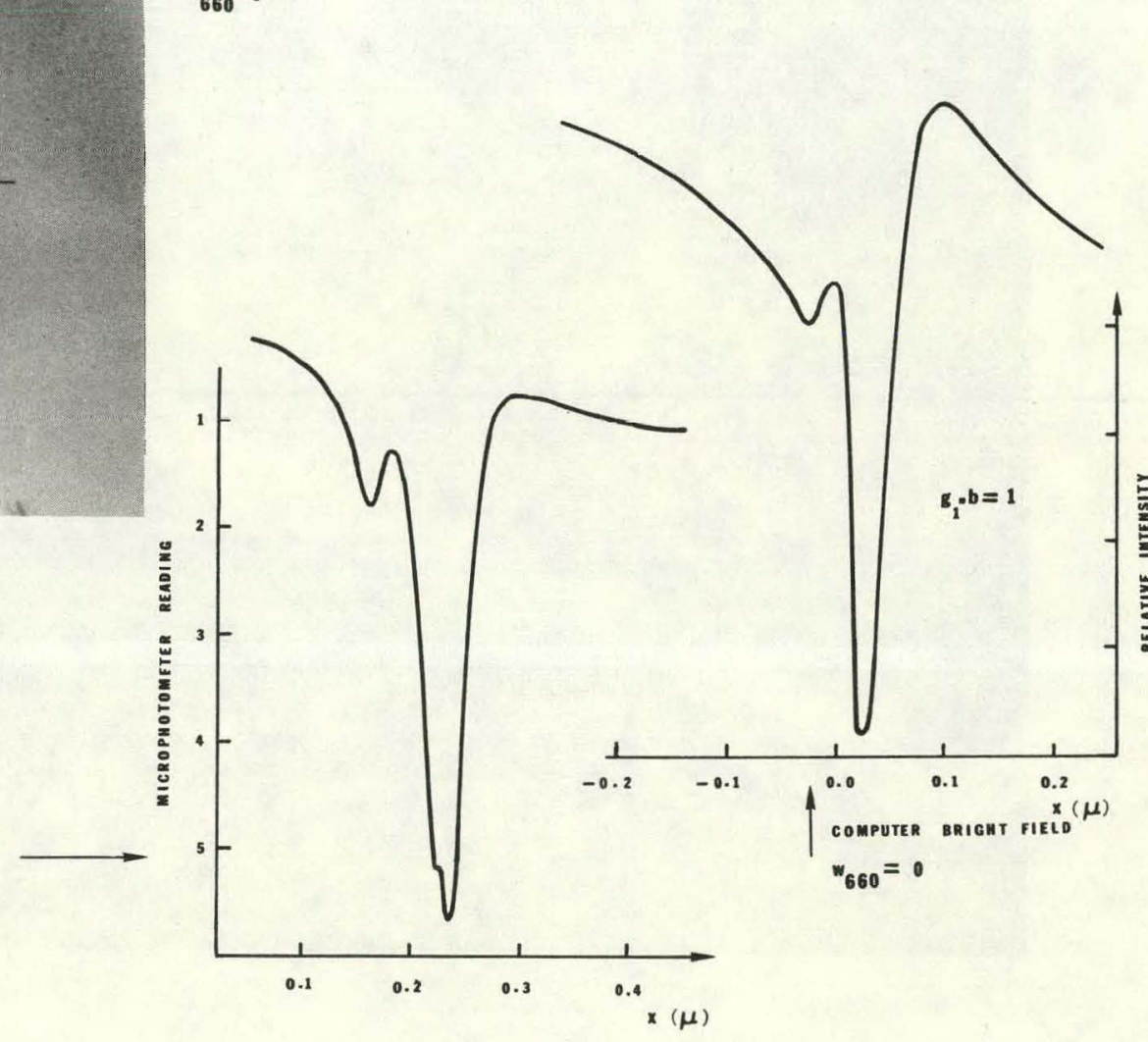

XBB707-3329

Figure 16 


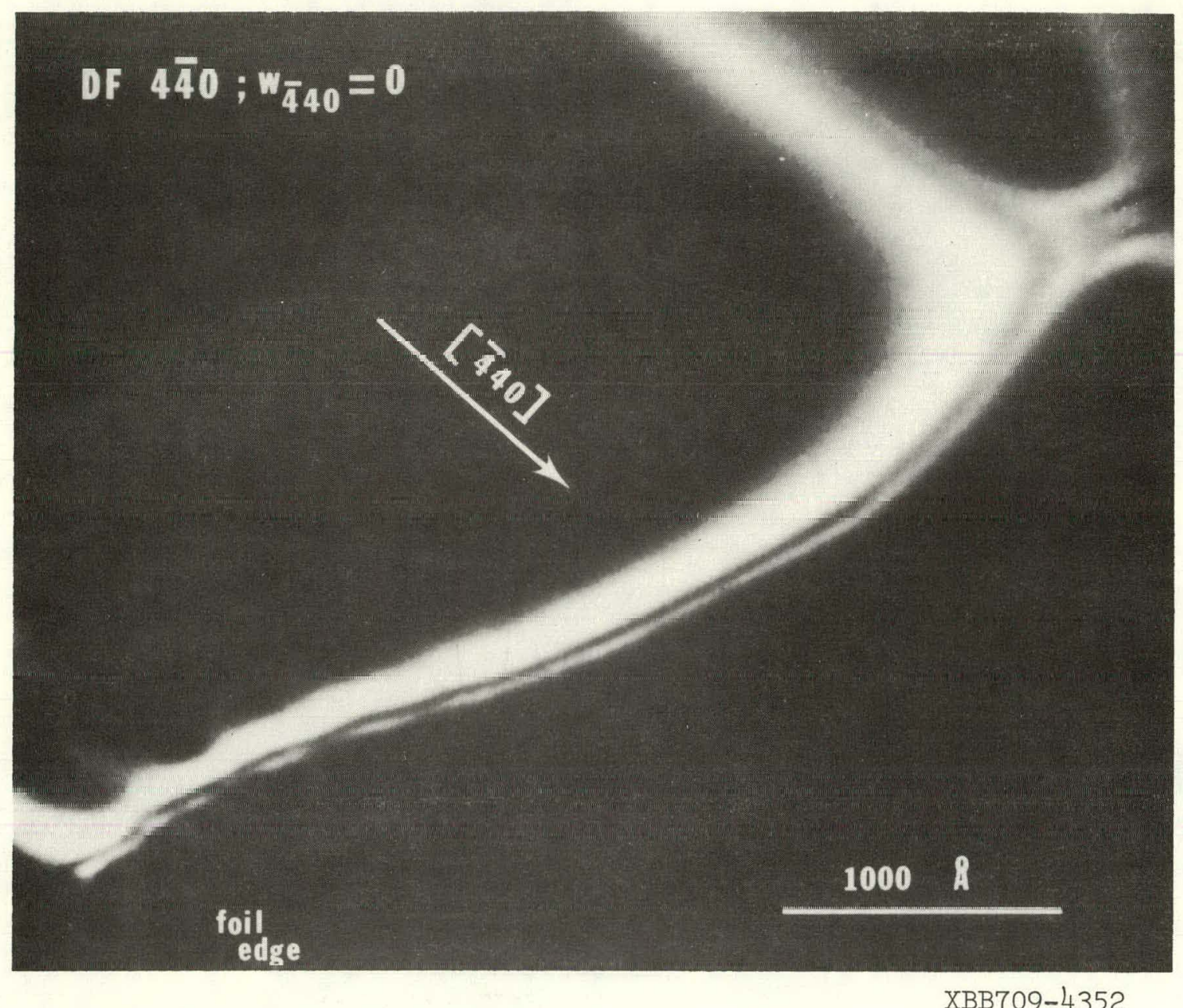

Figure 17 


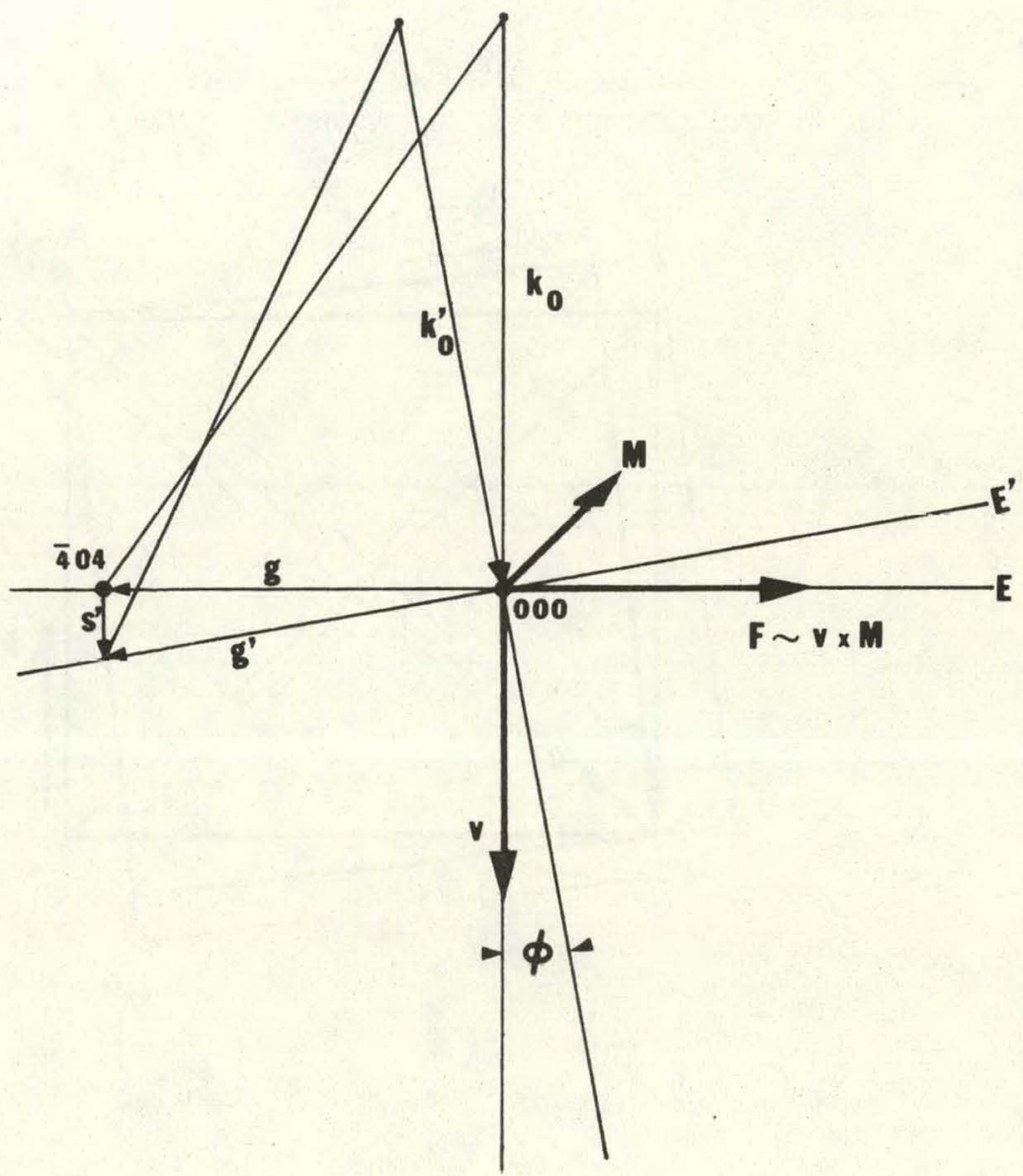

XBL 702-445

Figure 18 


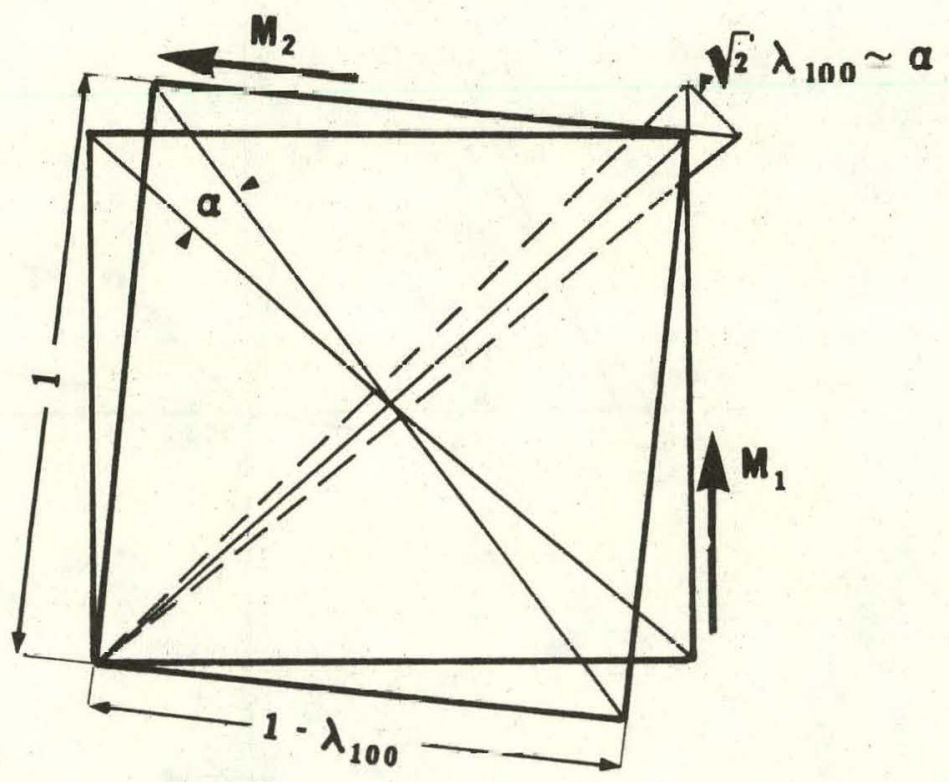

XBL 702-446

Figure 19 


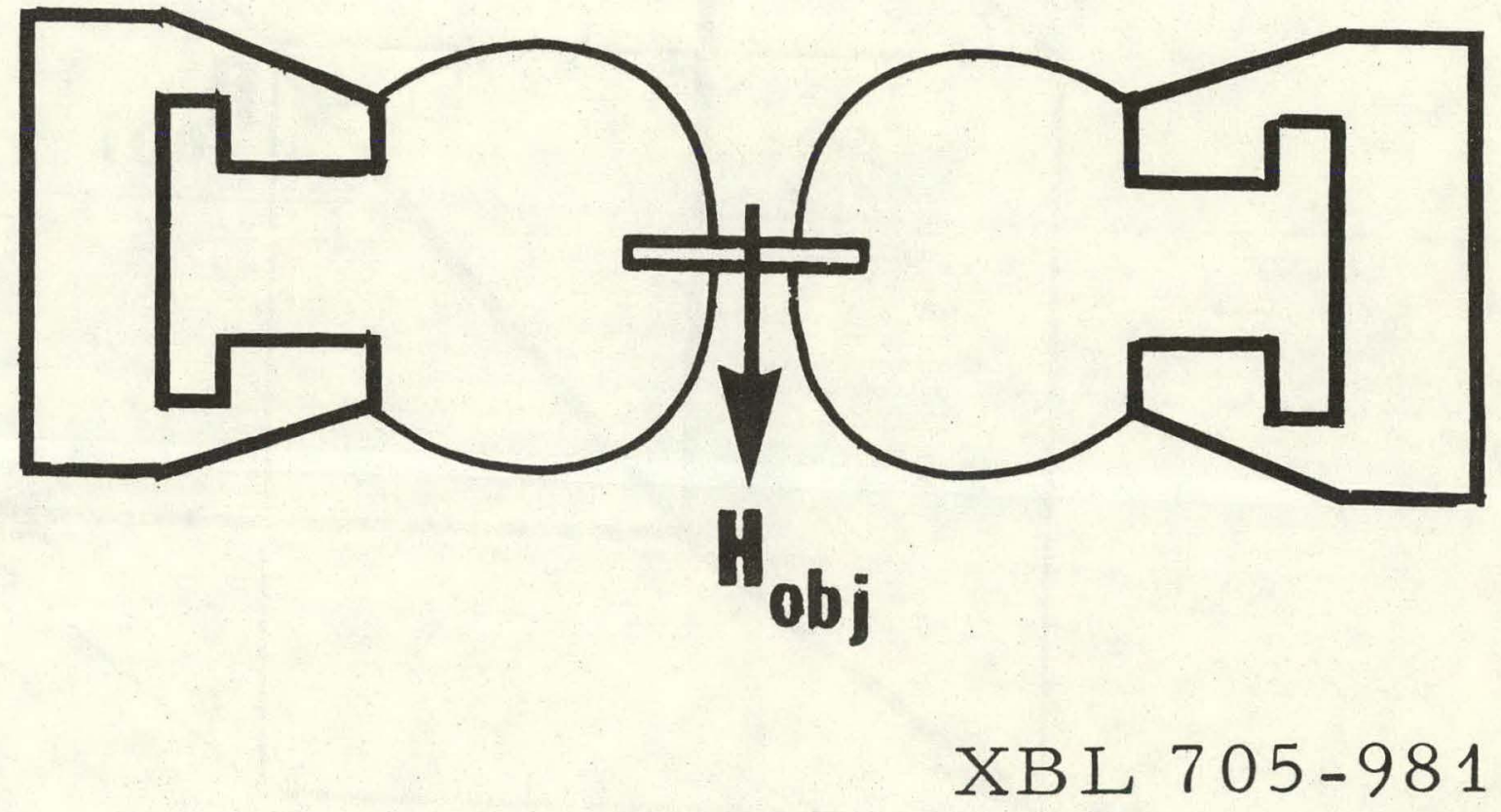

Figure 20 


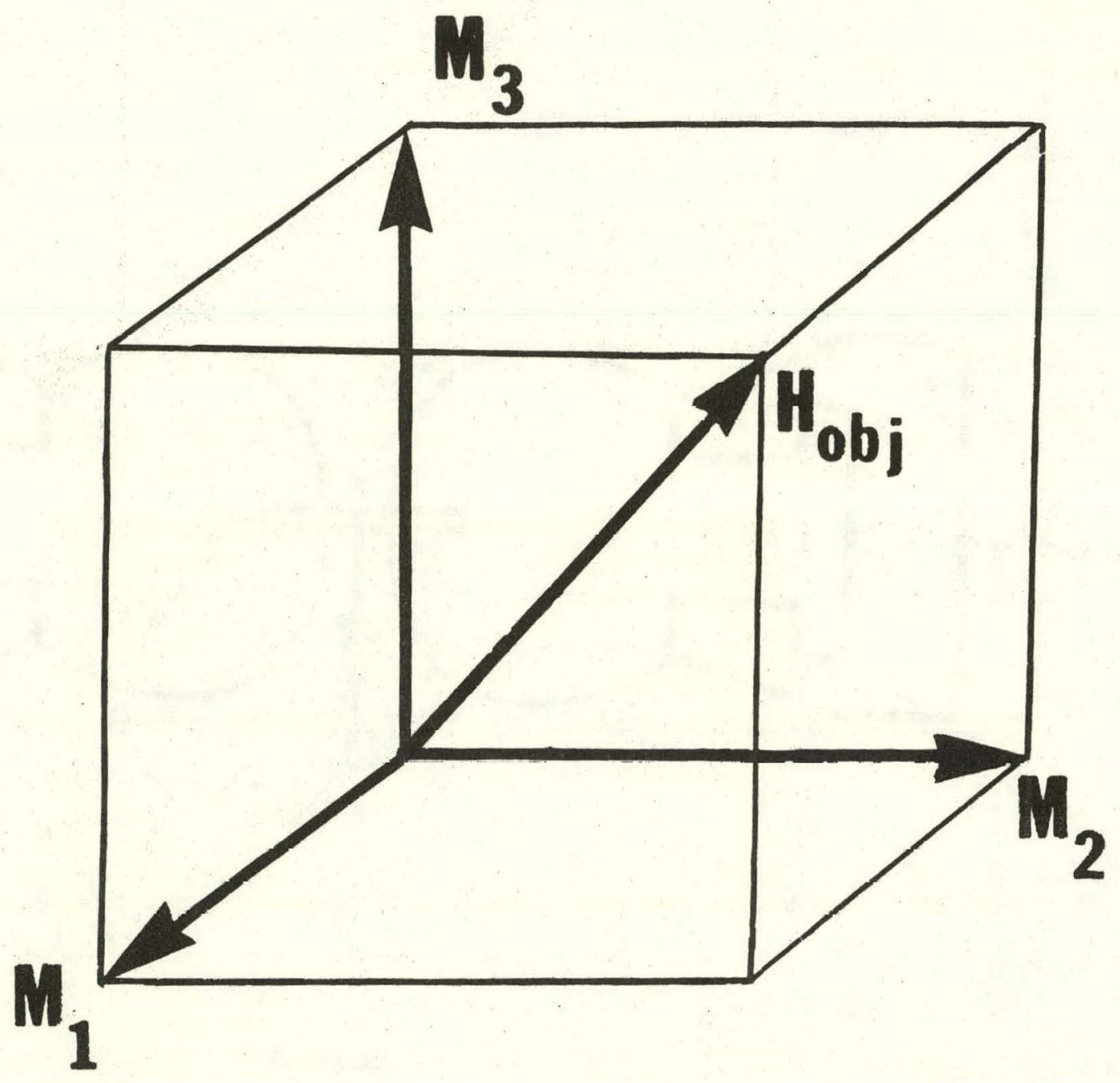

XBL 705-982

Figure 21 


\section{Ferrimagnetic domains}

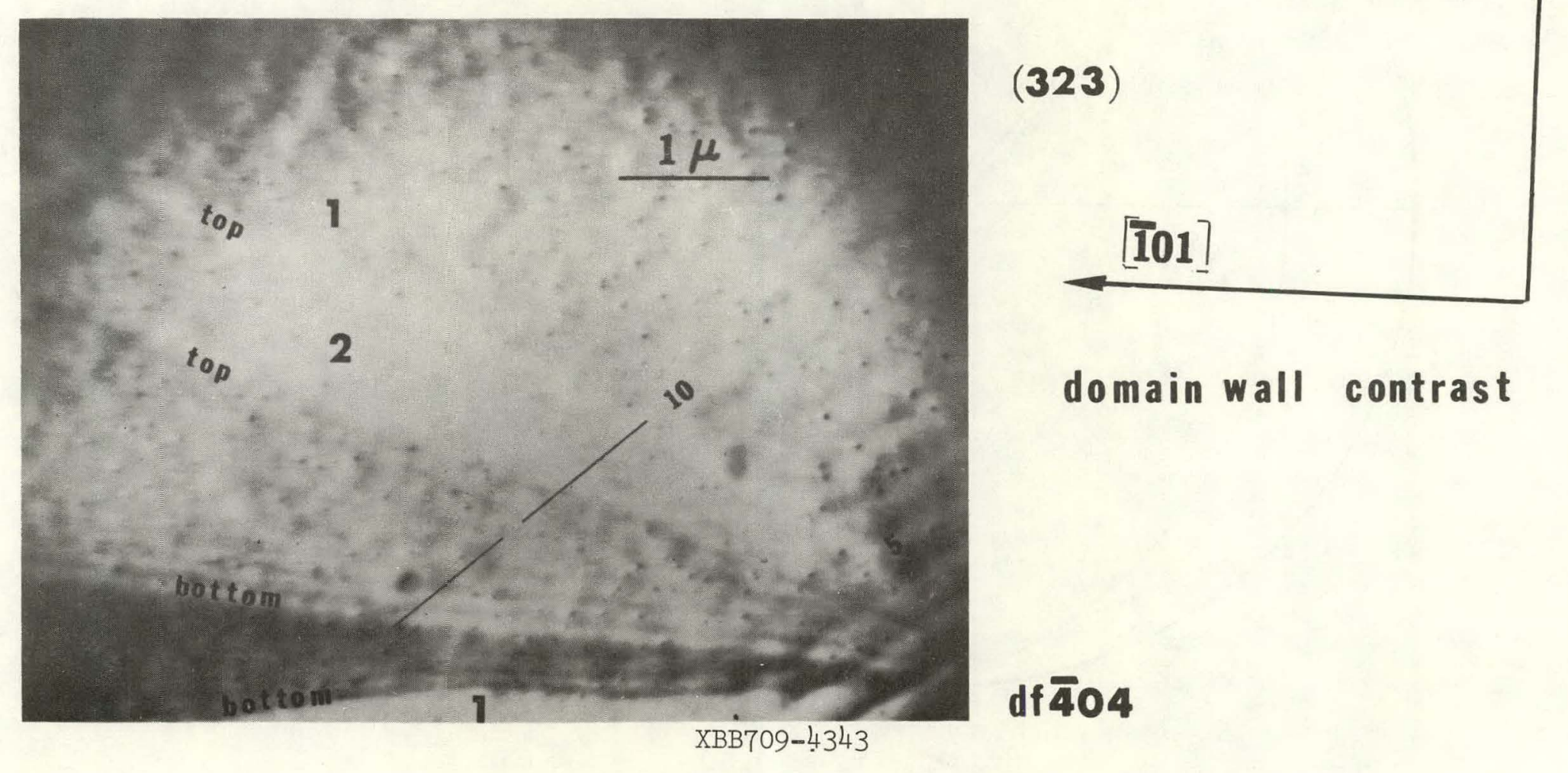




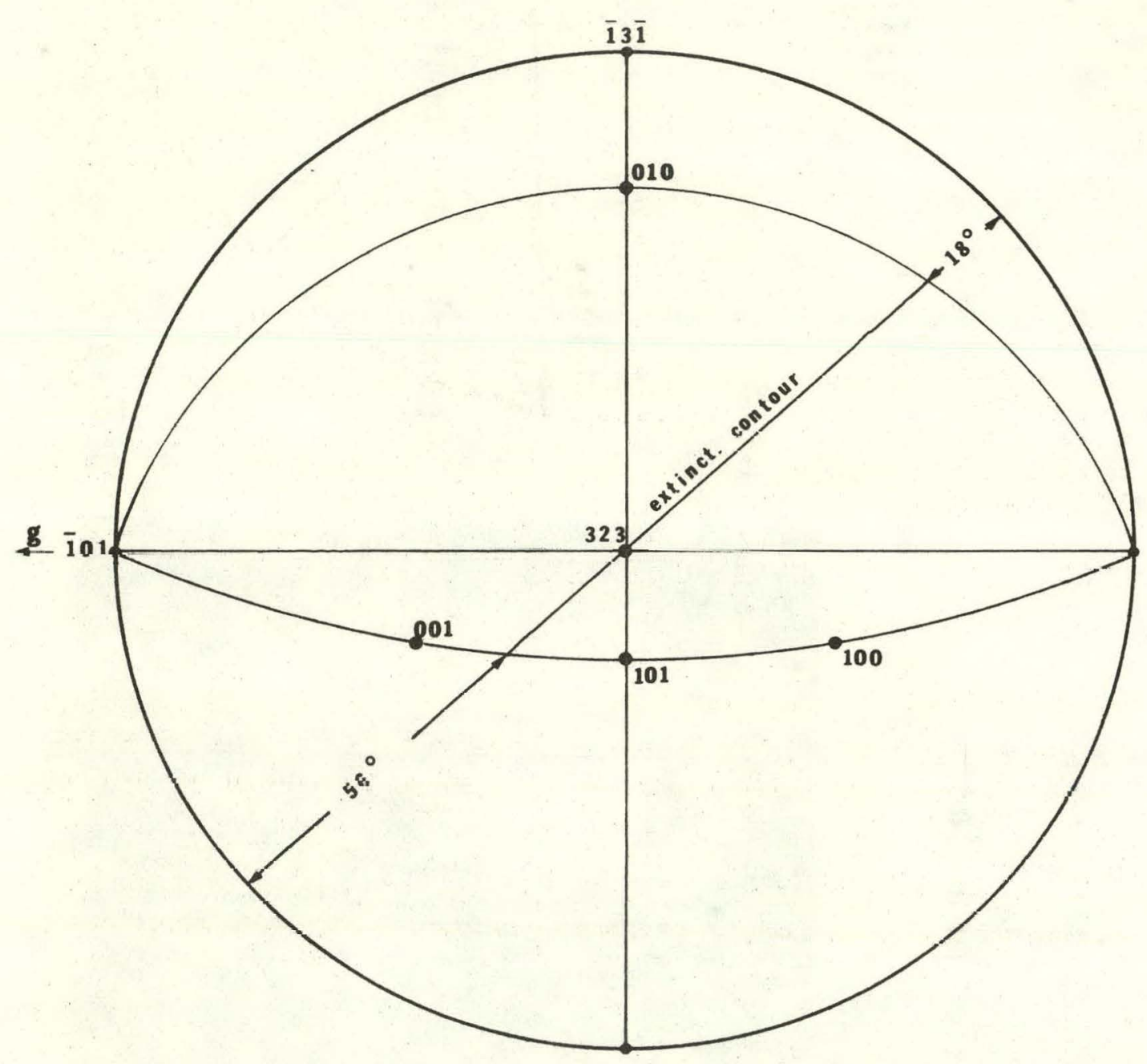

XBL 709-6520

Figure 23 

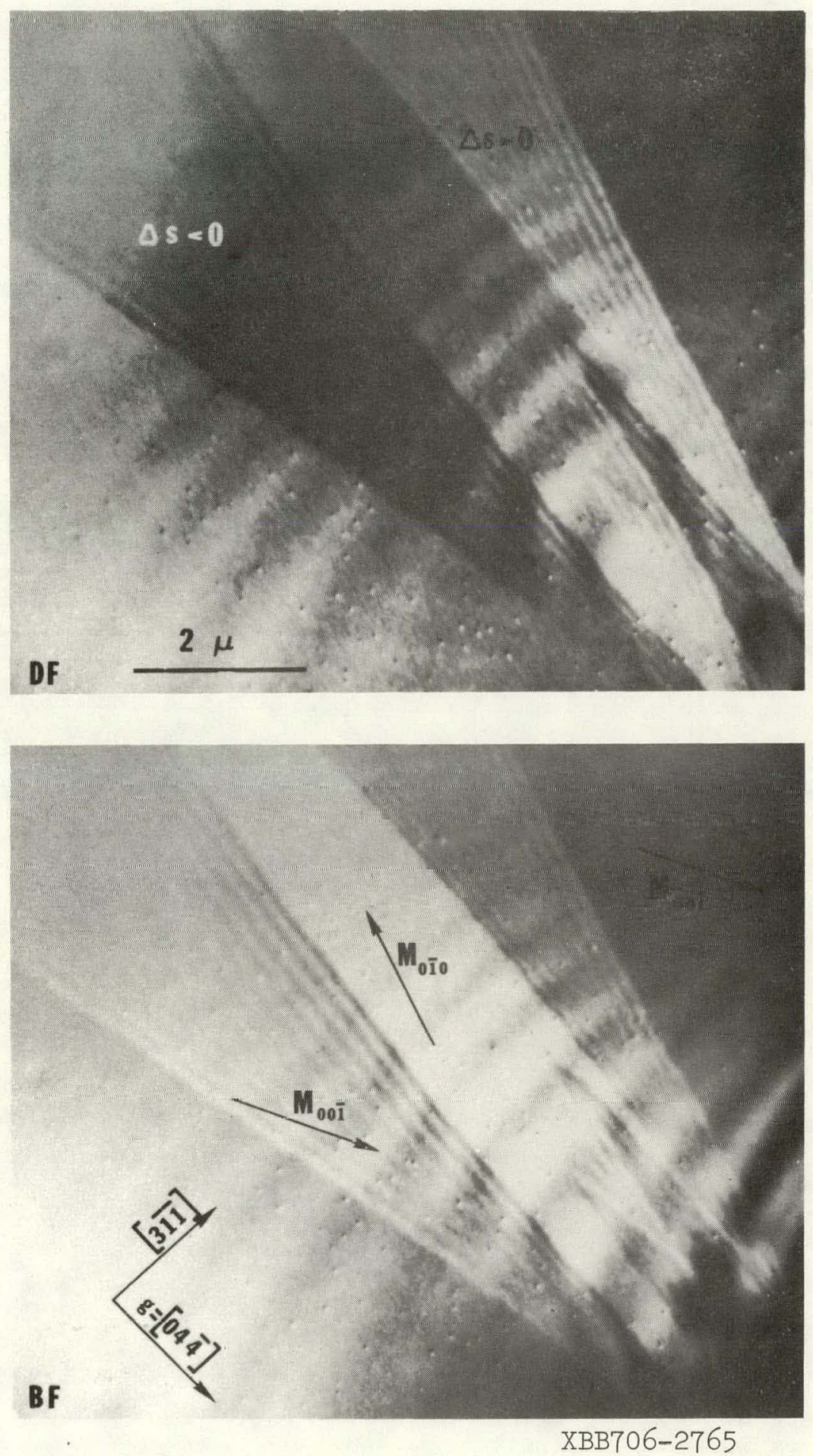

Figure 24 


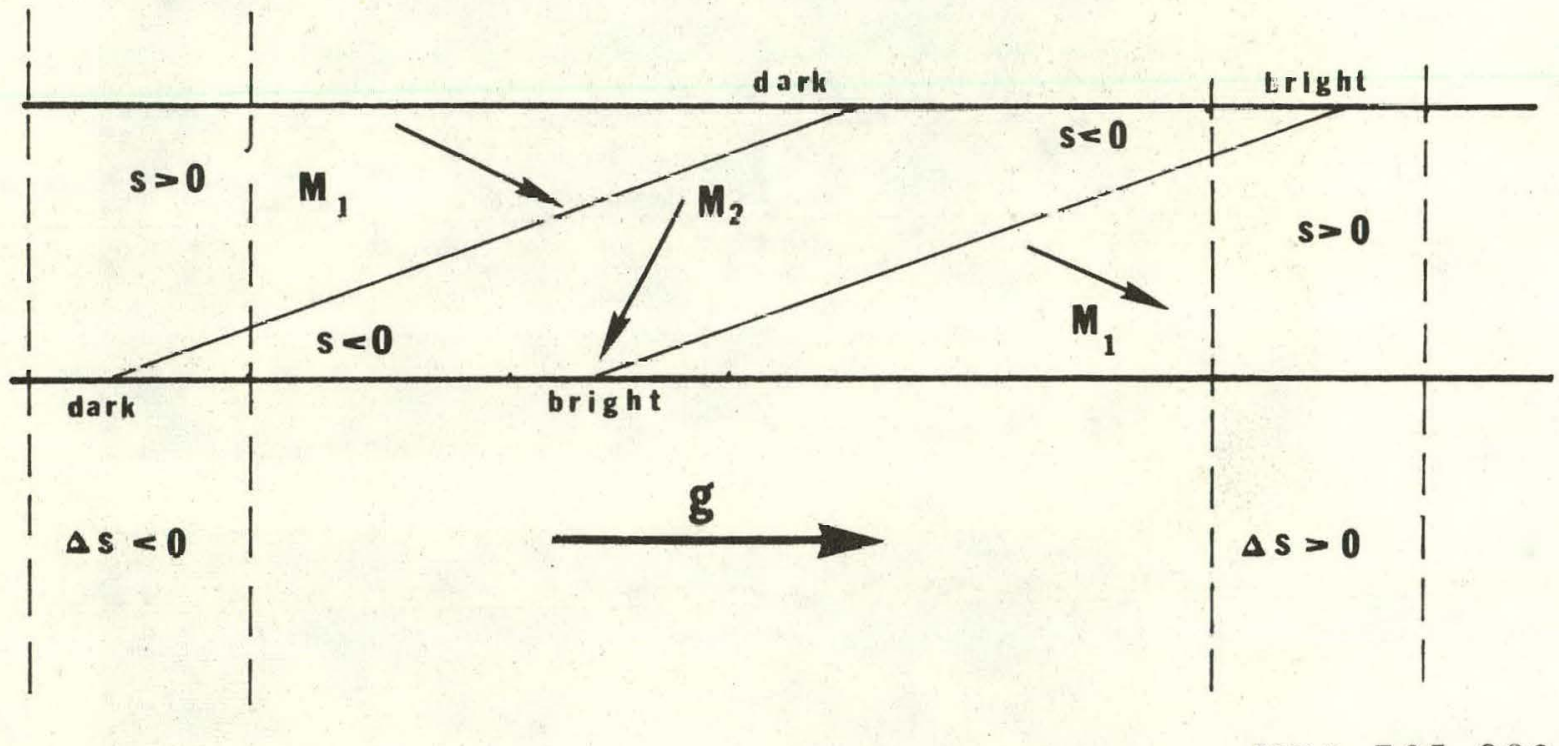

XBL 7 05-983

Figure 25 
-103-

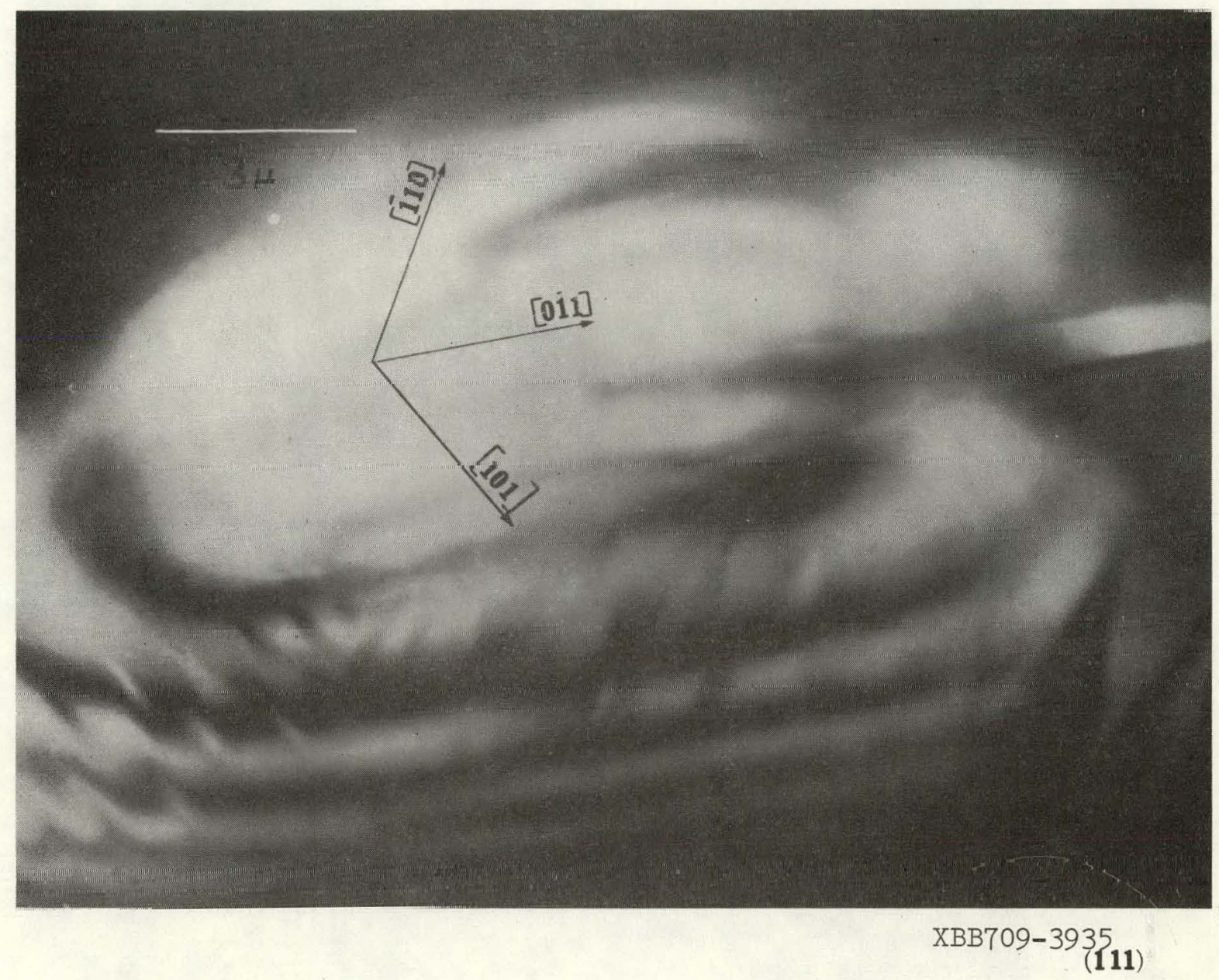

Figure 26 

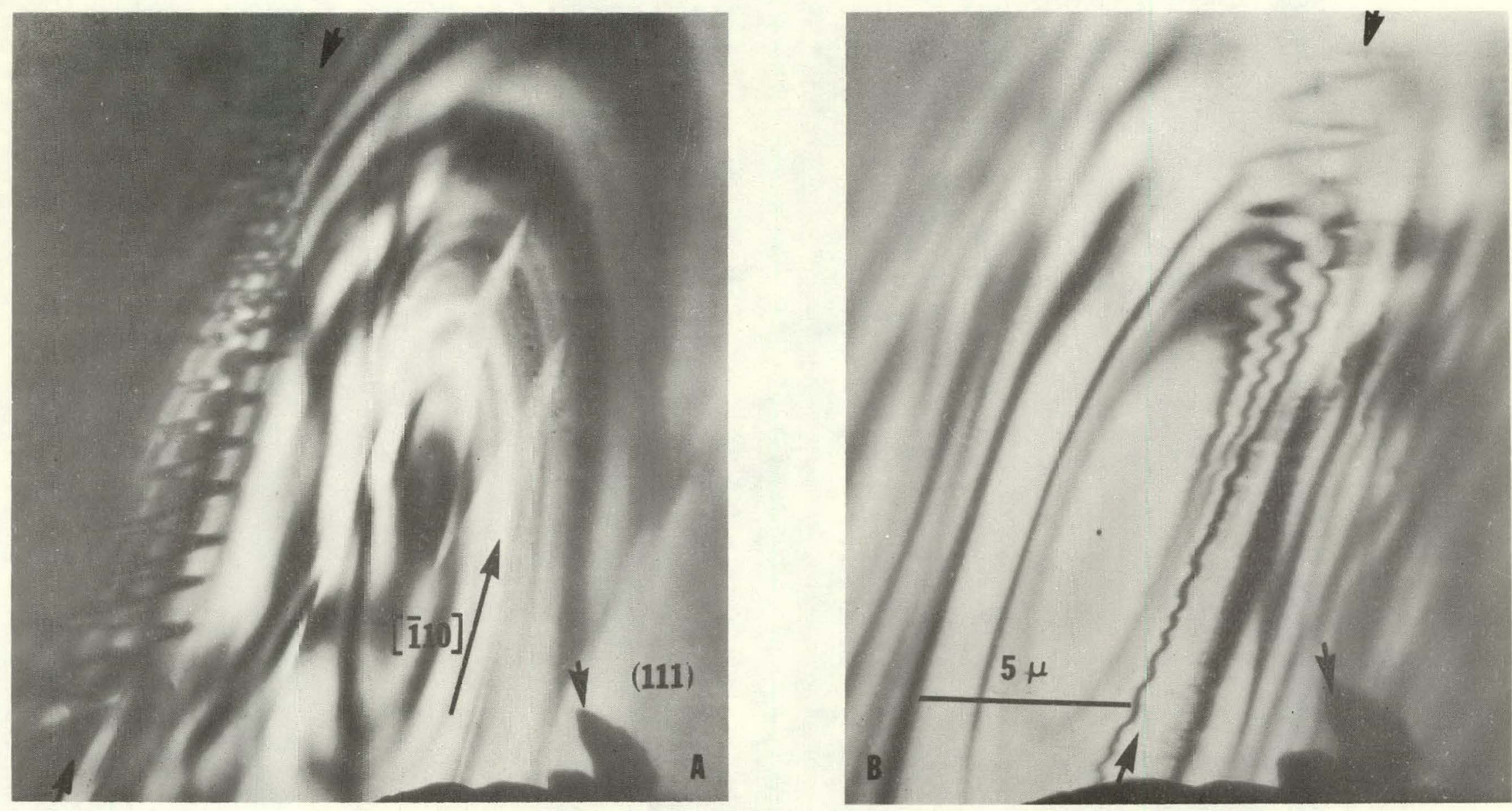

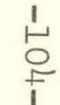

Figure 27

XBB709-3938 
$-105-$

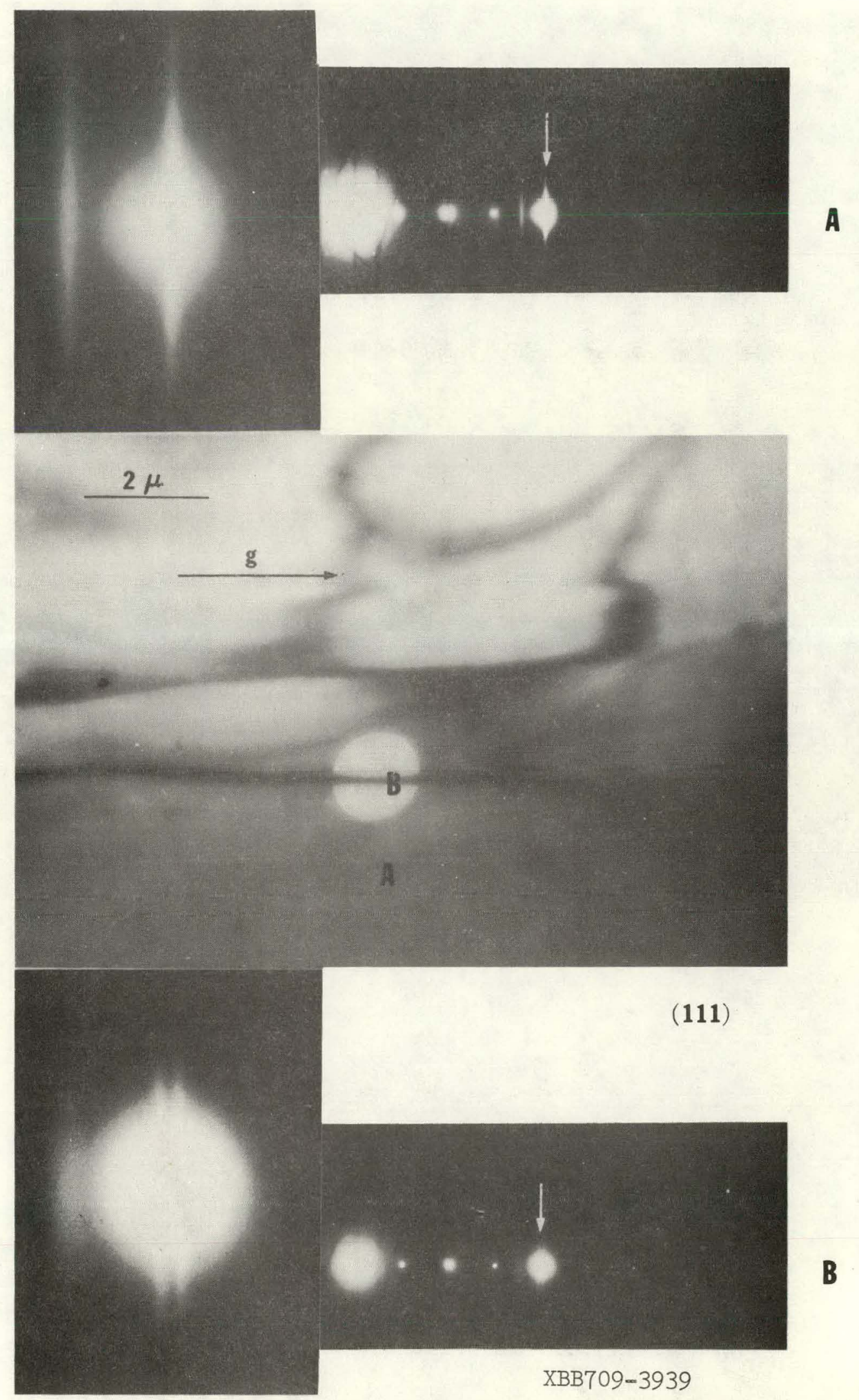


$-106-$

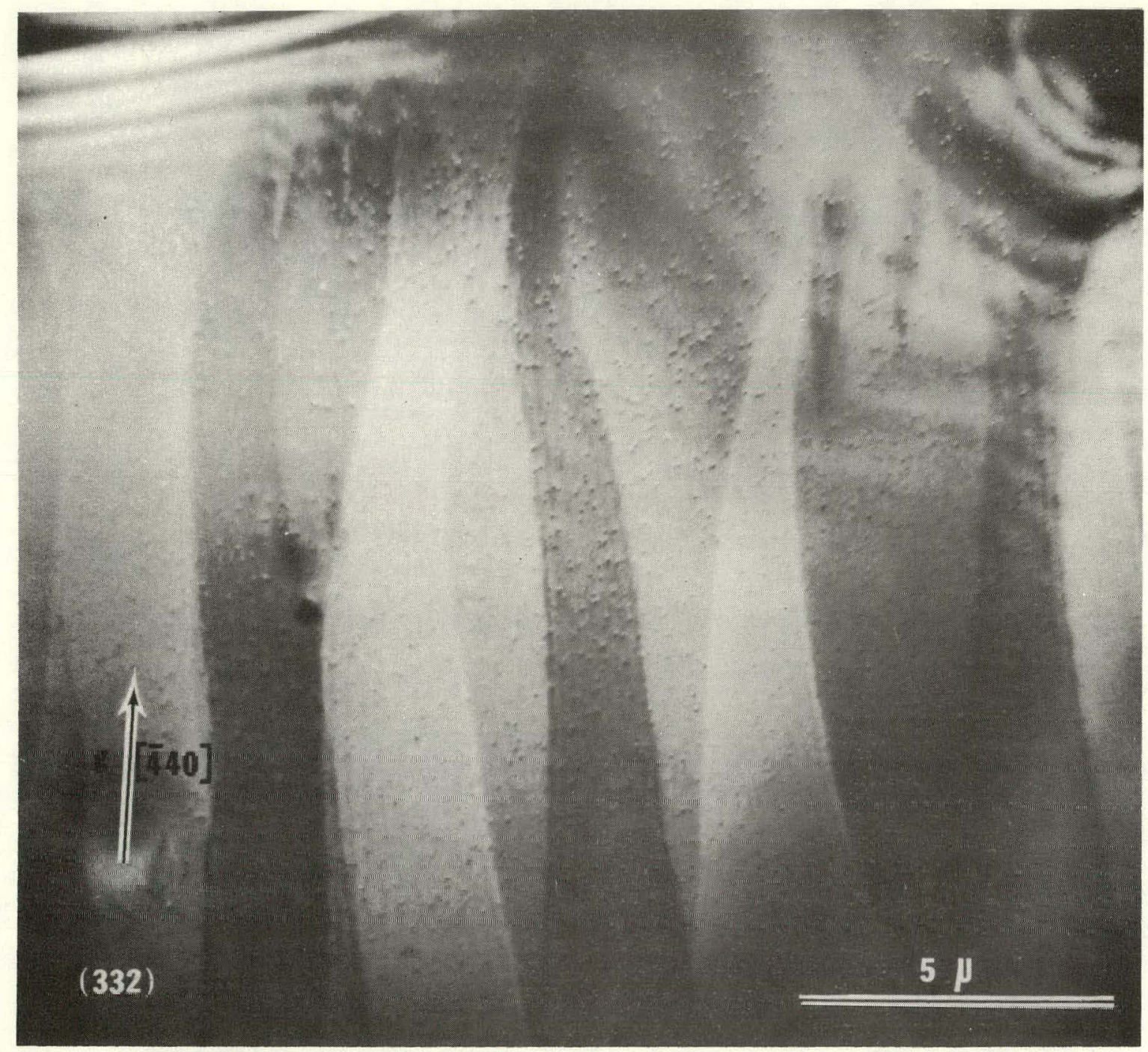

XBB706-2767

Tigure 29 

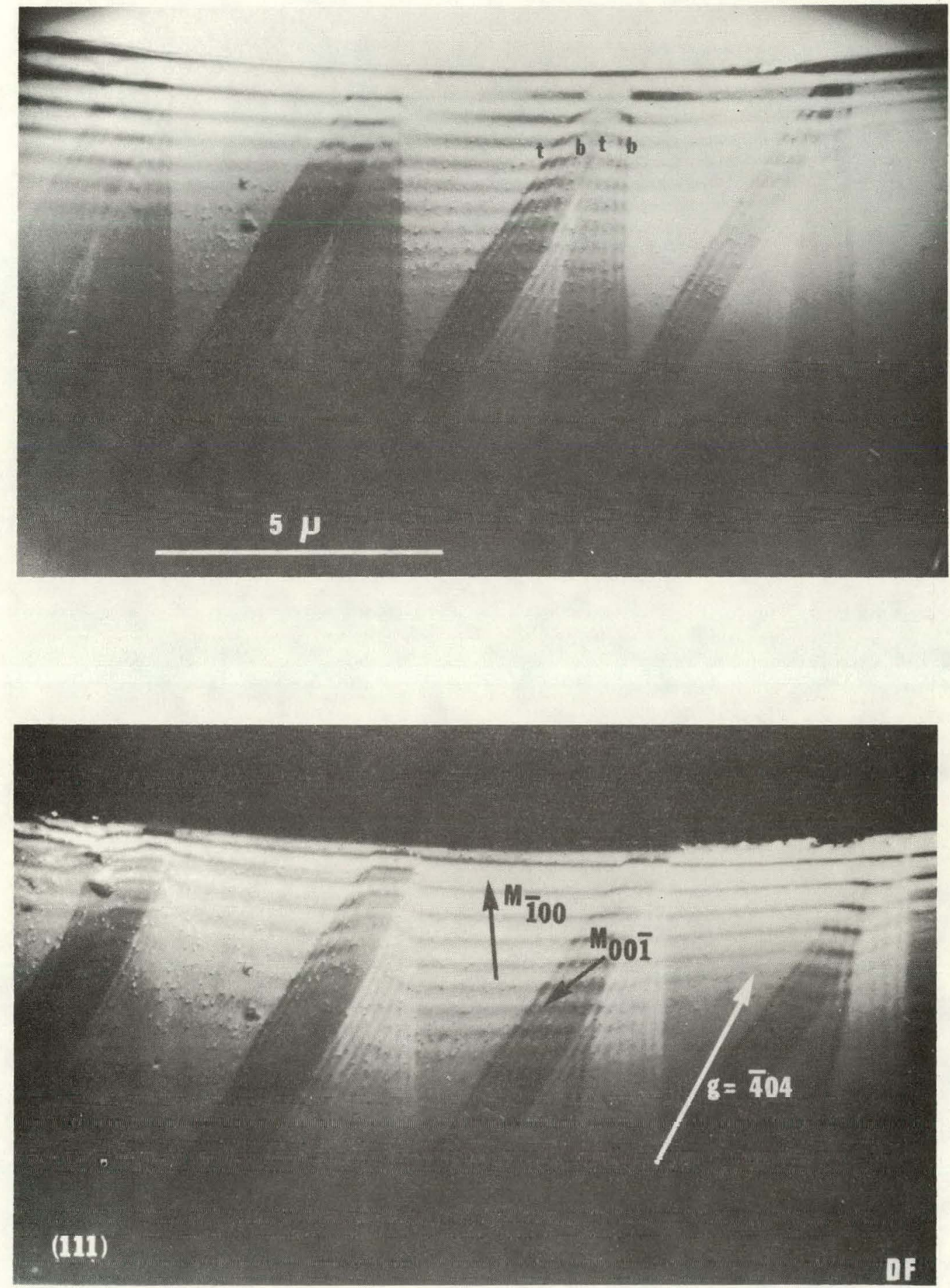

XBB706-2766

Figure 30 


$$
-108-
$$

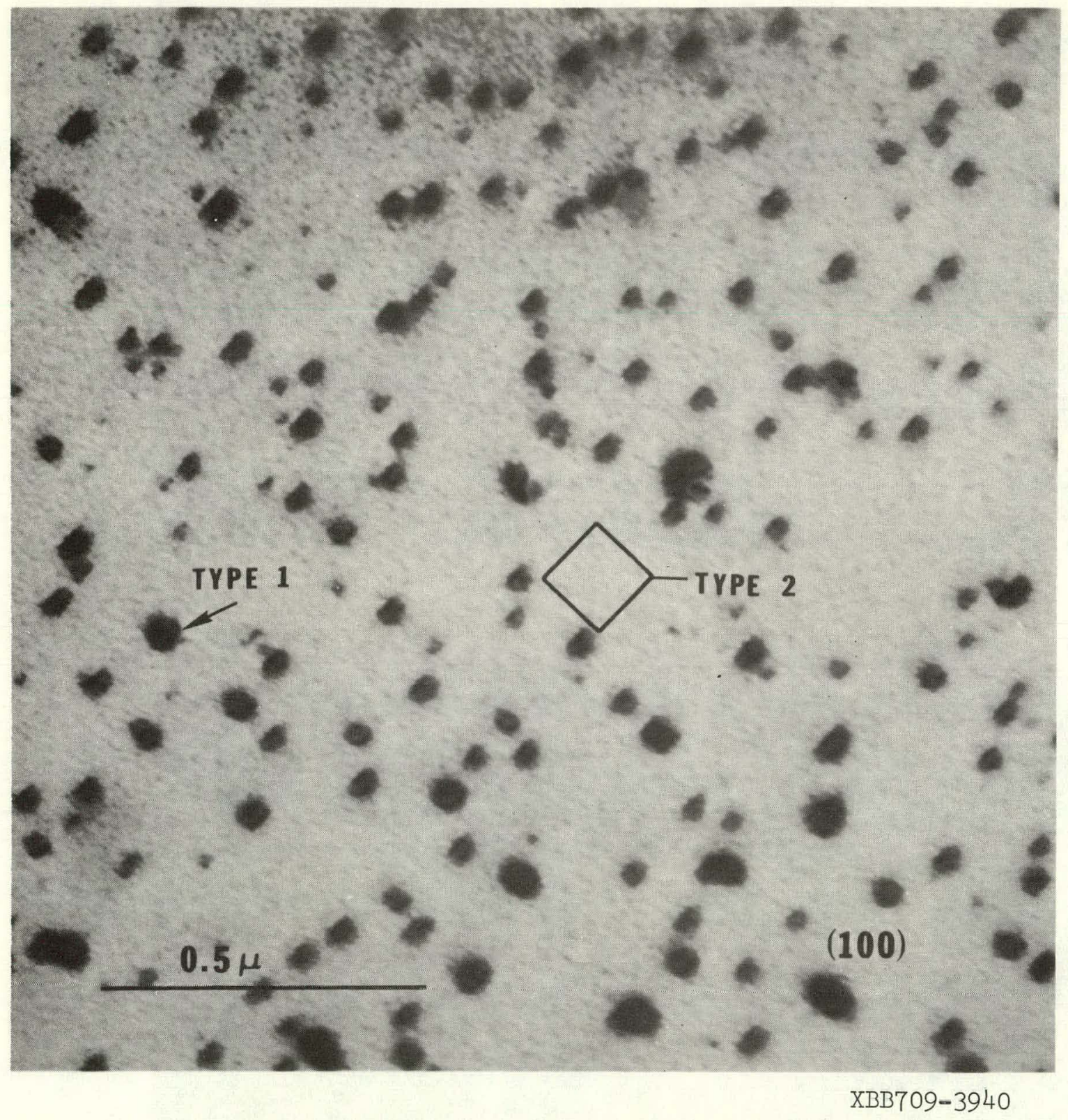

Figure 31 


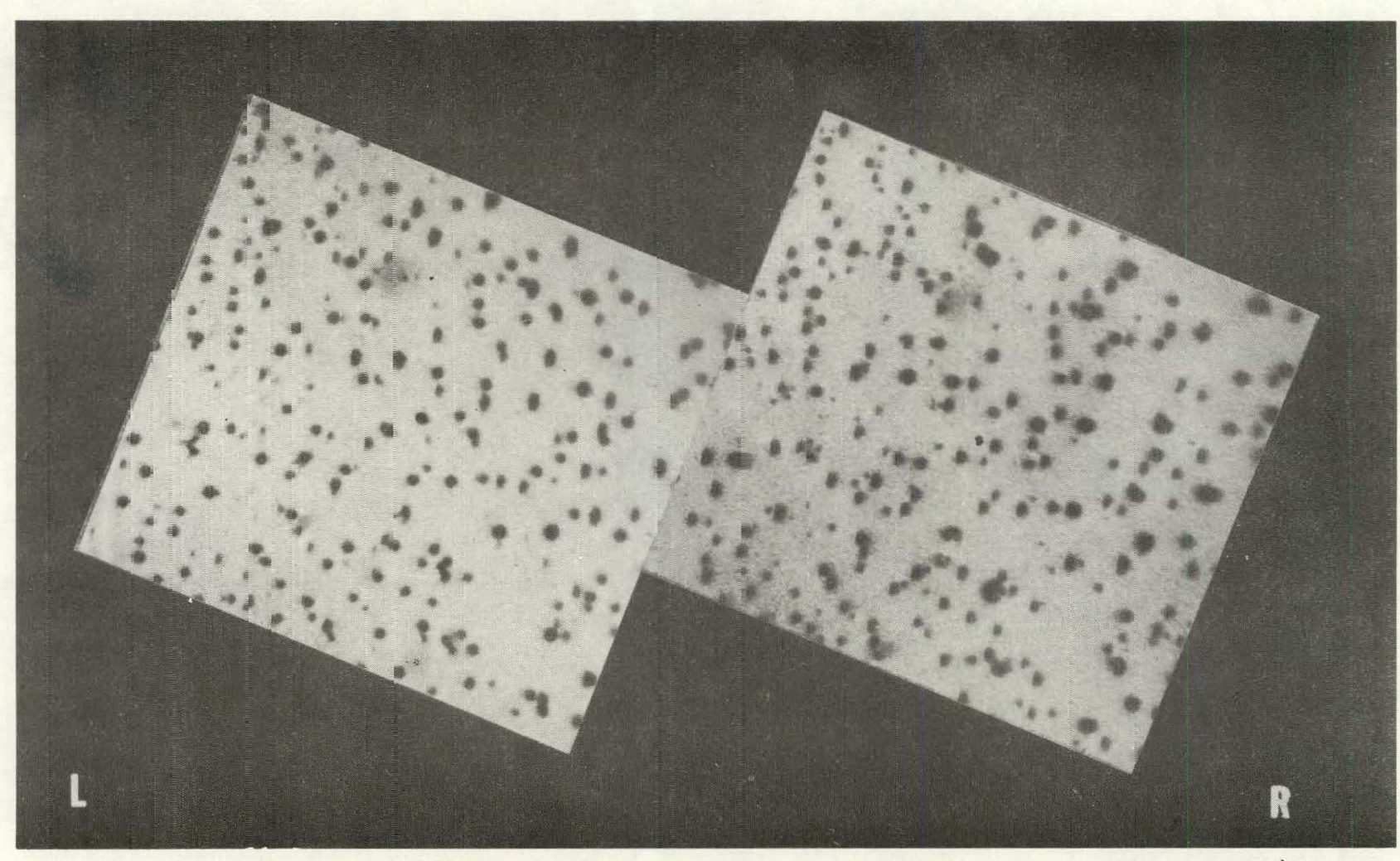

XBB709-3941

Figure 32 


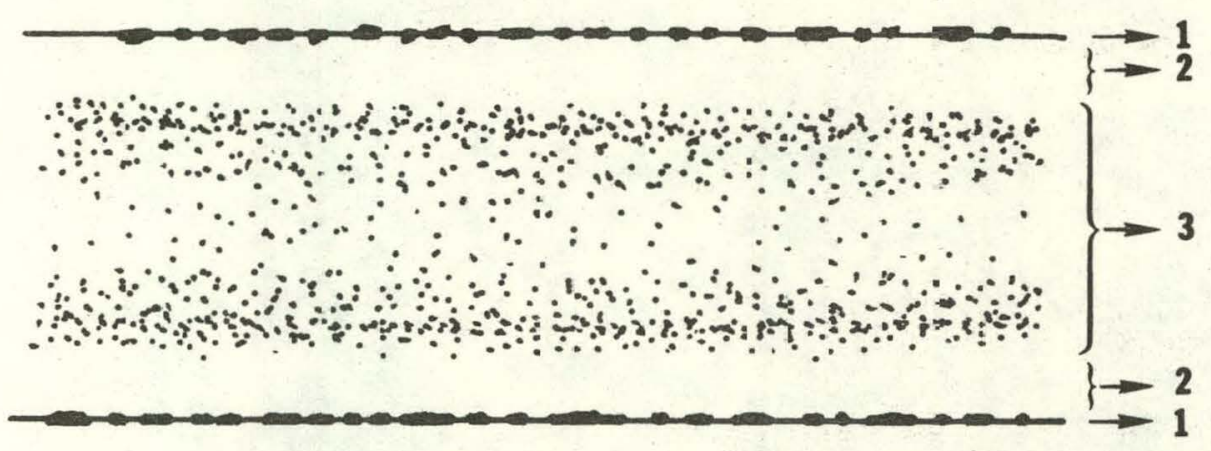

1- type I precipitates in surface layer

2-precipitate free region

3- inner reaction zone with

XBL 709-6569

Figure 33 


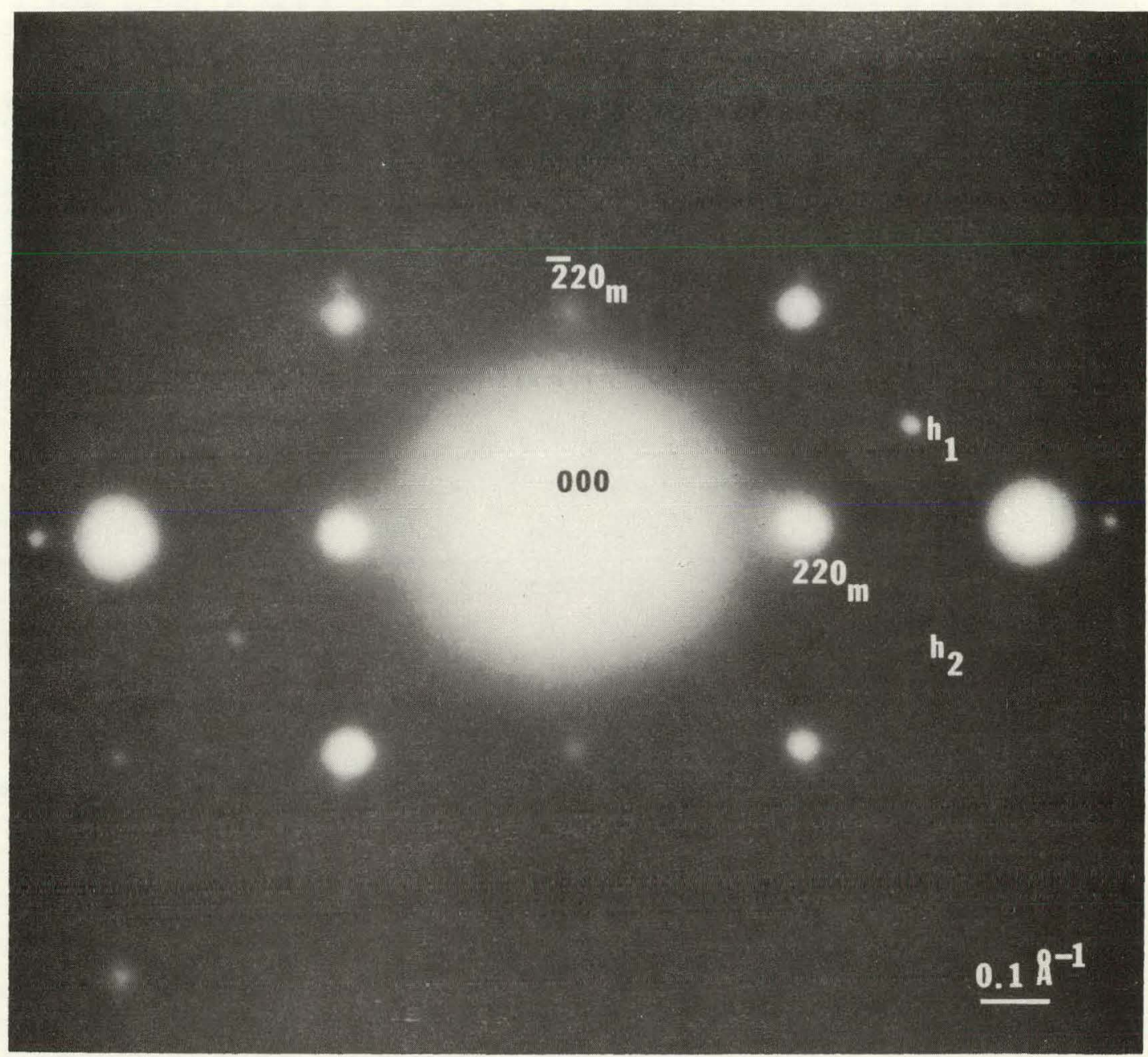

XBB709-4015

Figure 34a 


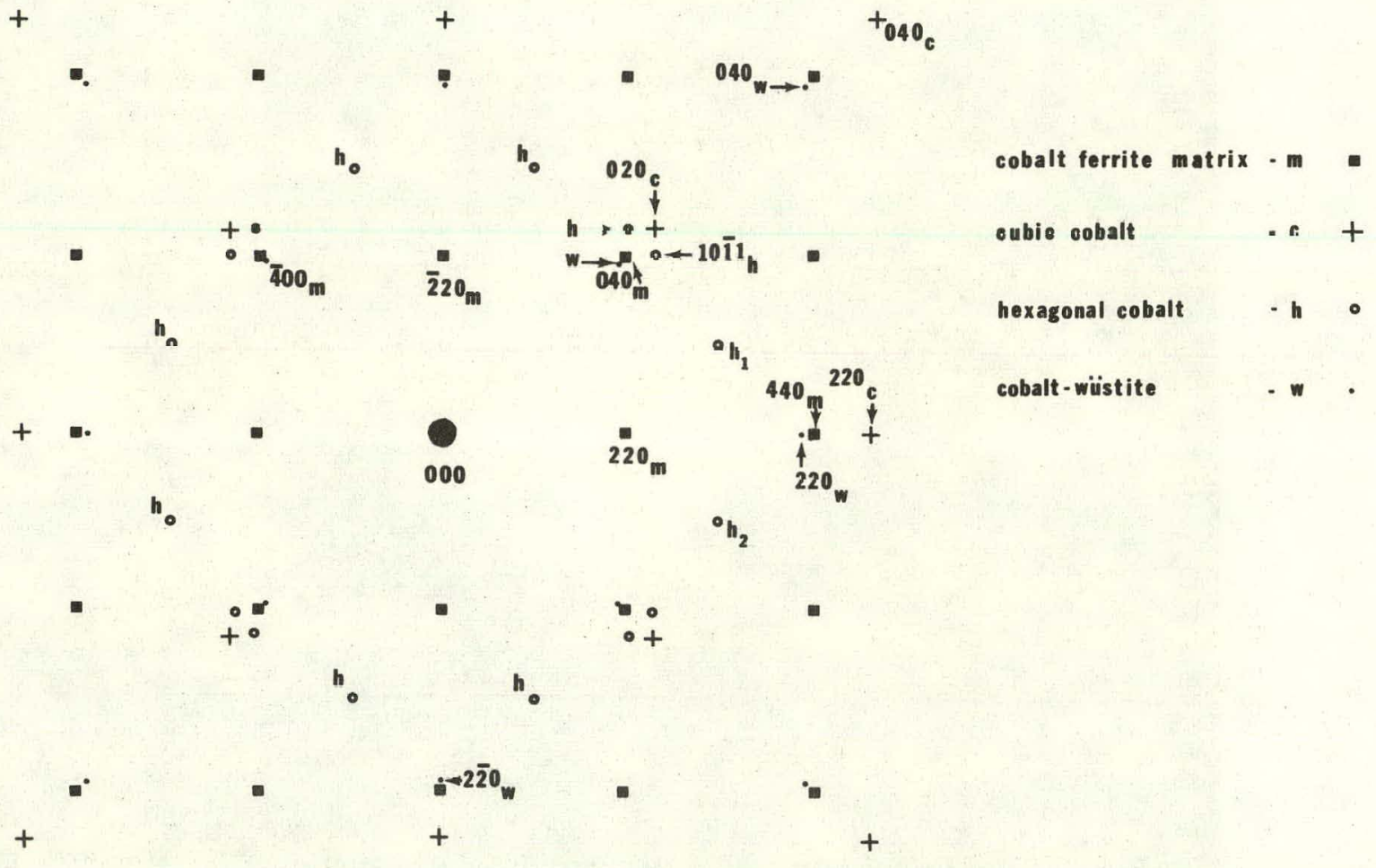

Figure 34b 


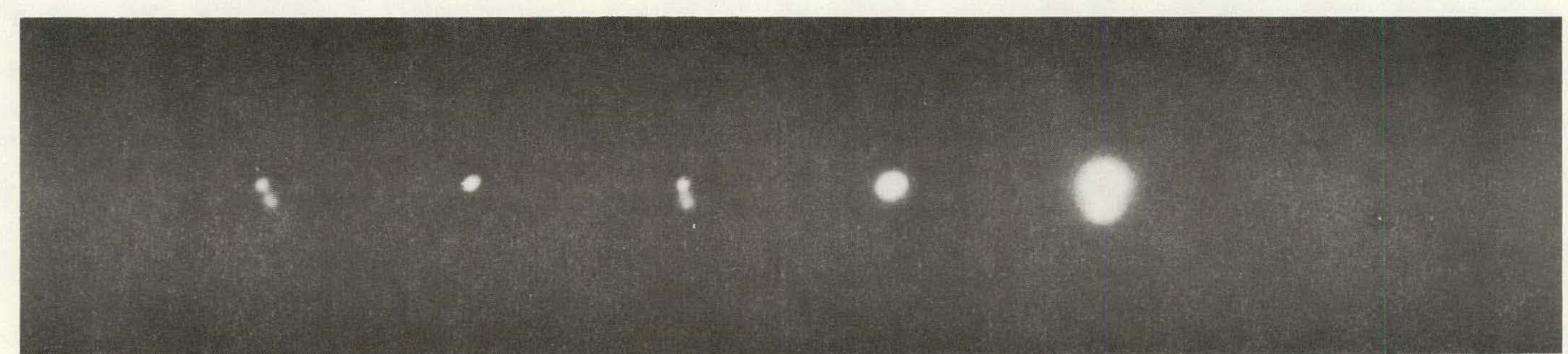

XBB709-4009

$(1 \overline{4} 5)$

$10^{-3} \mathrm{rad}$

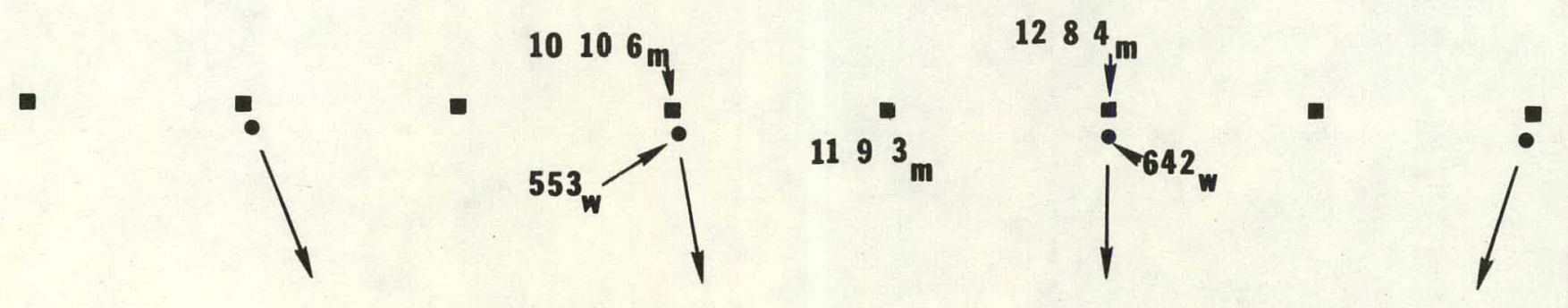

Figure 35 


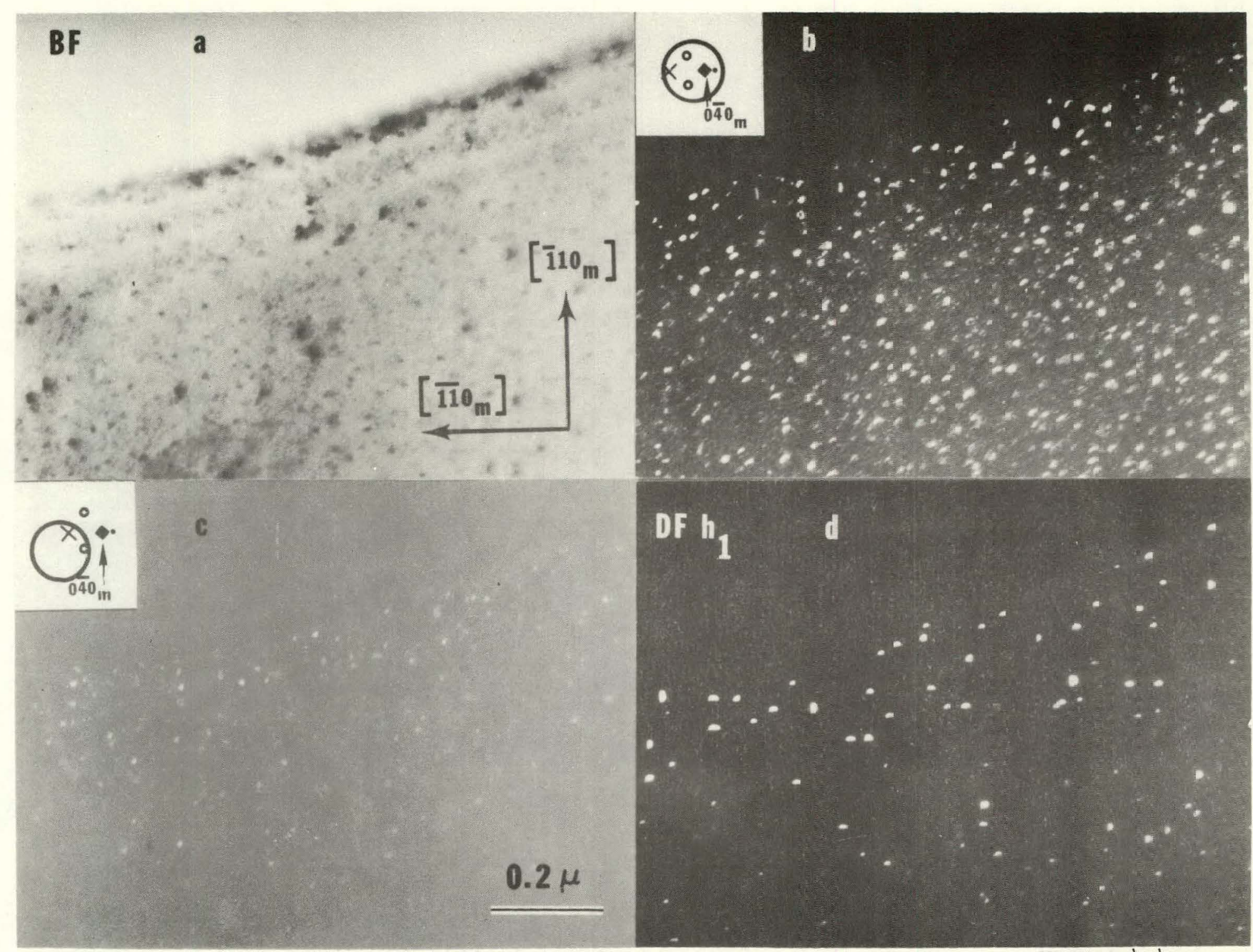

Figure 36 


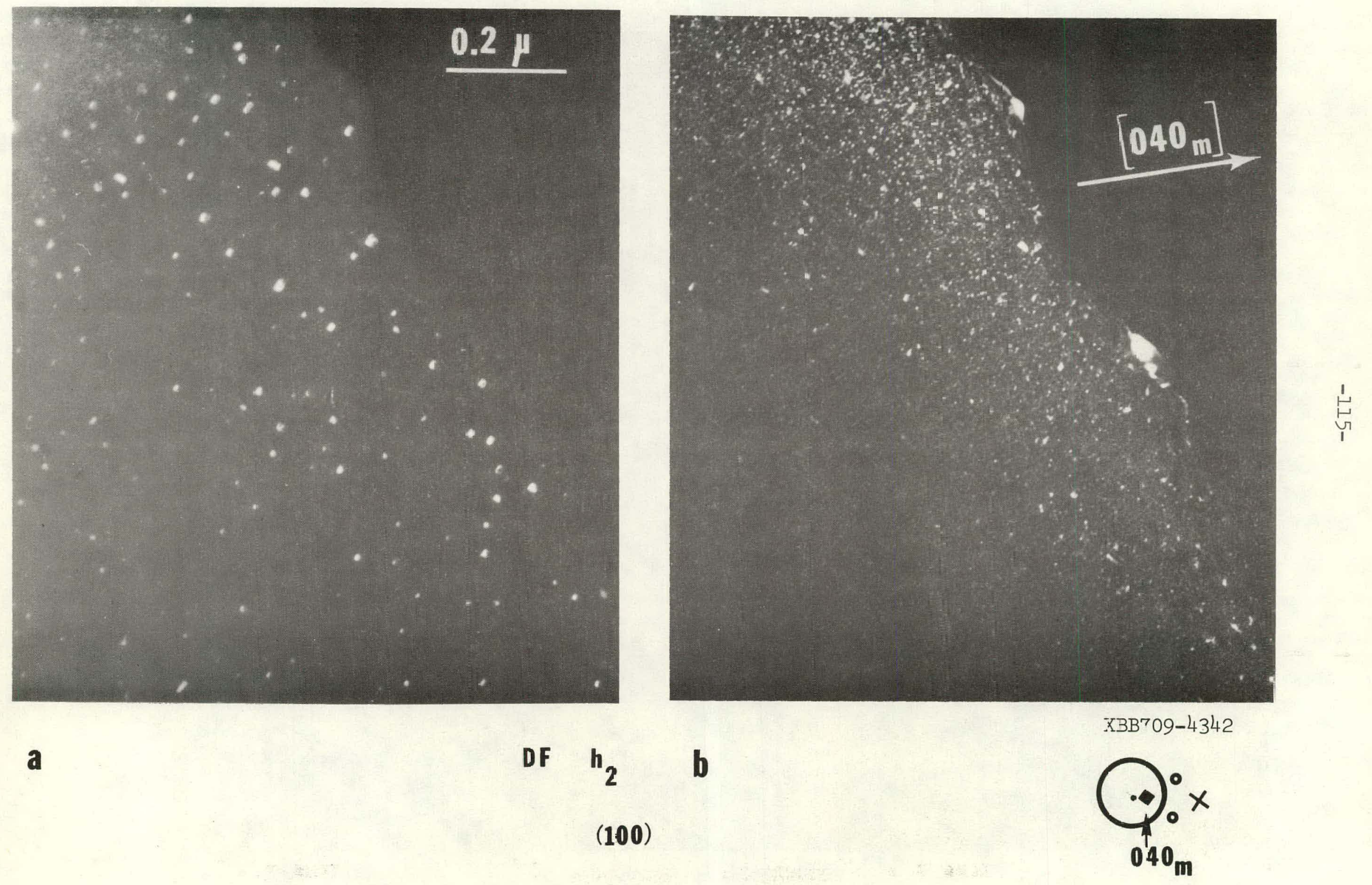

Iigure 37 


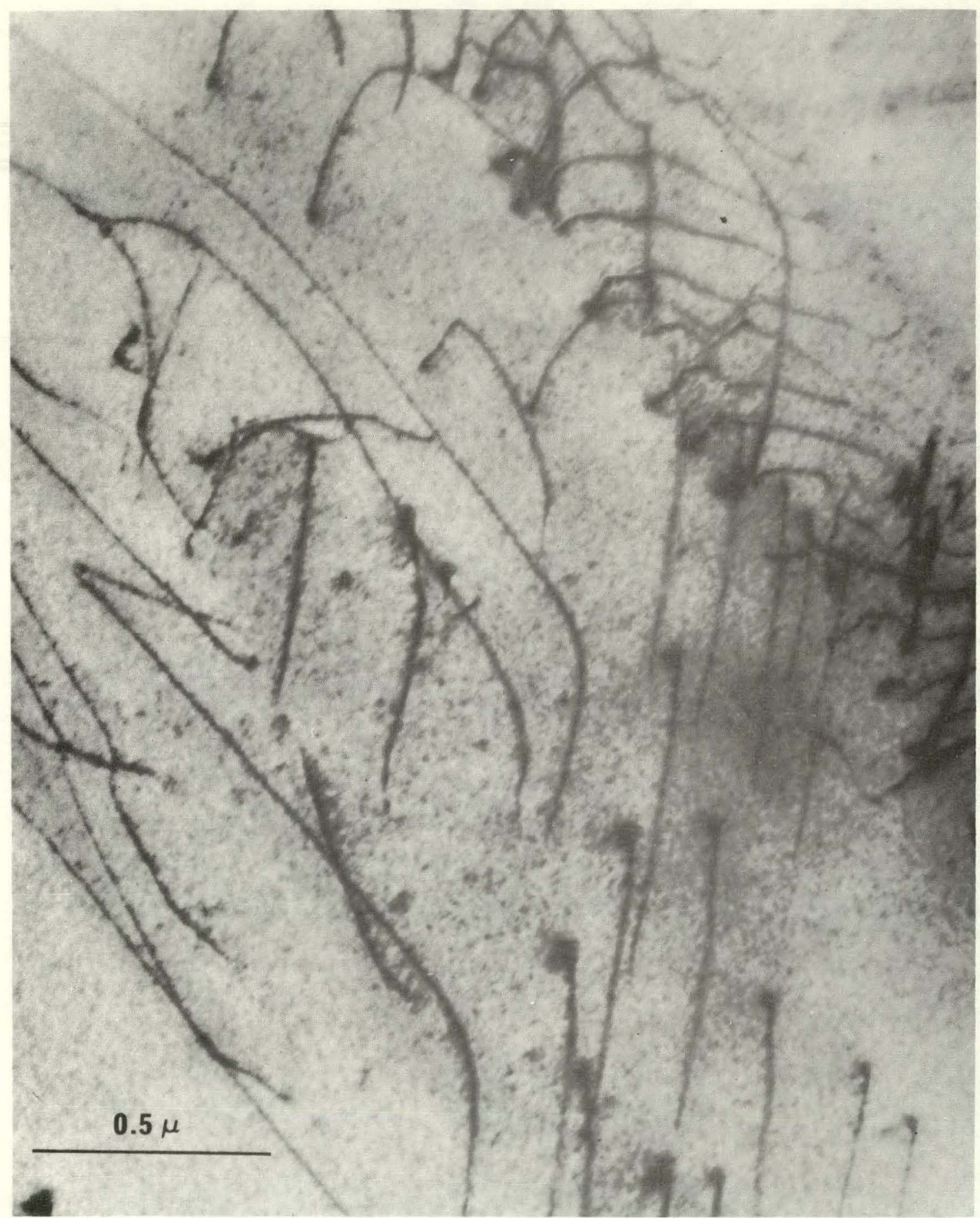

XBB709-3934

Figure 38 

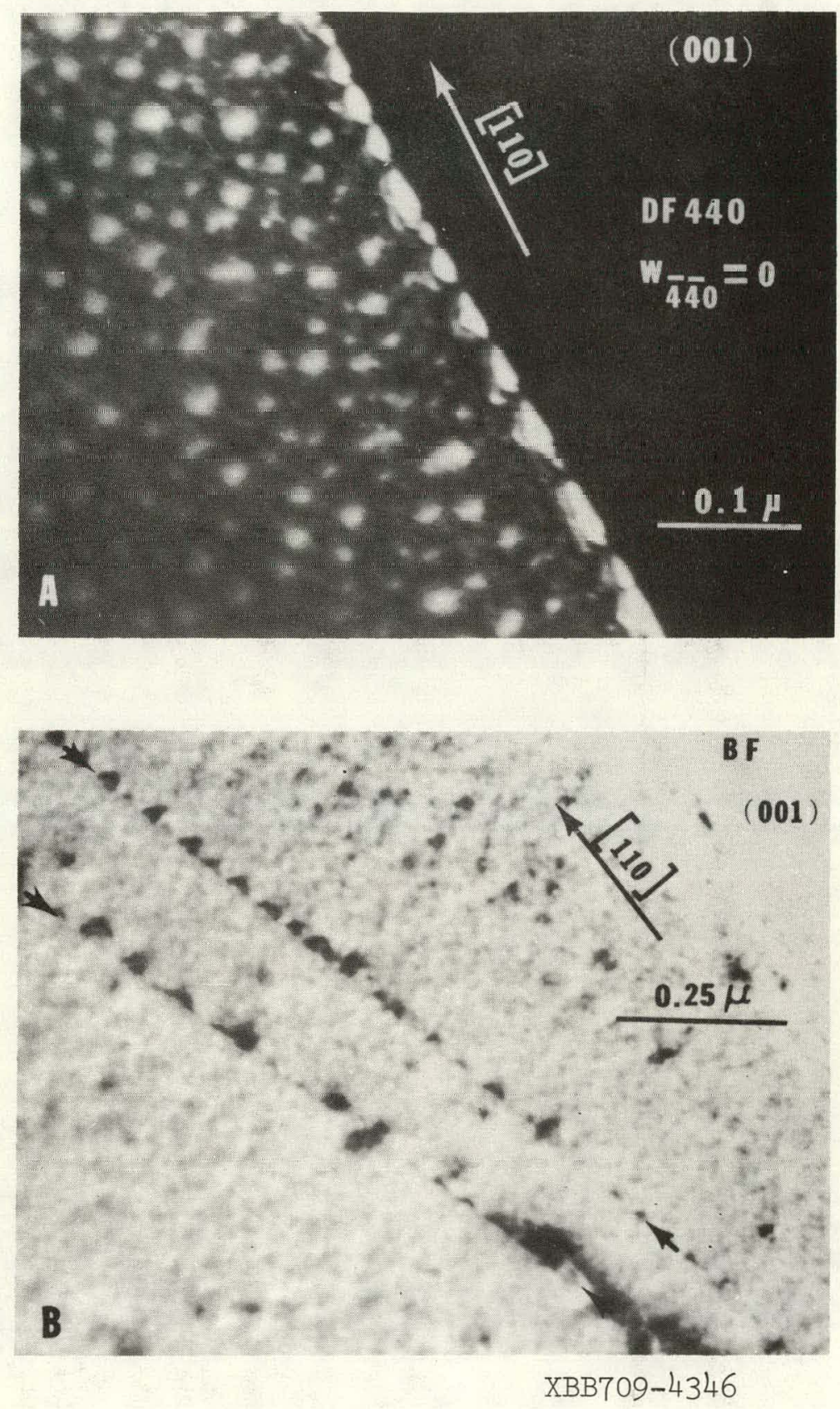

Figure 39 

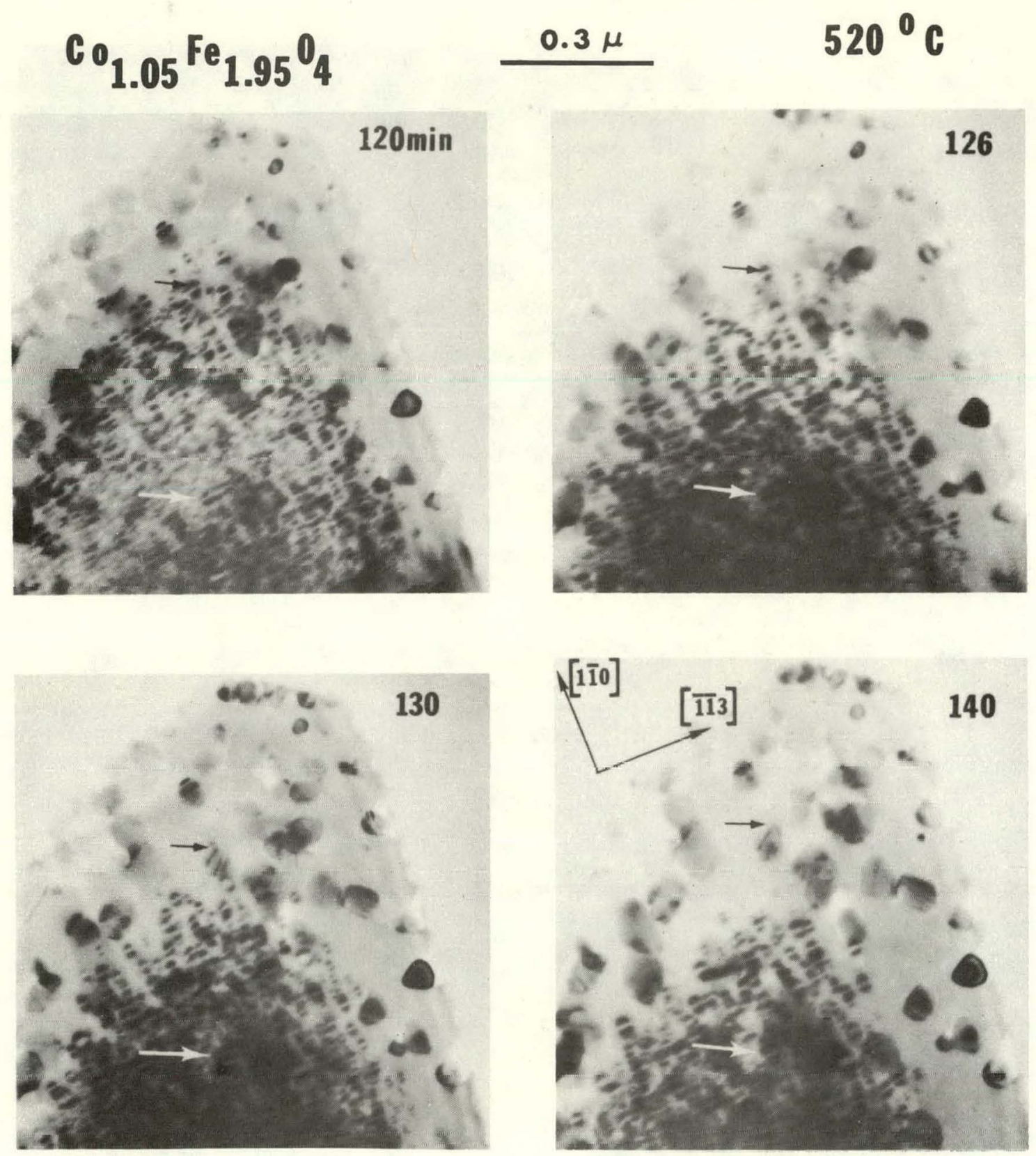

XBB70?-401?

Figure 40 


$$
\mathrm{Co}_{1.2} \mathrm{Fe}_{1.8} \mathrm{O}_{4}
$$
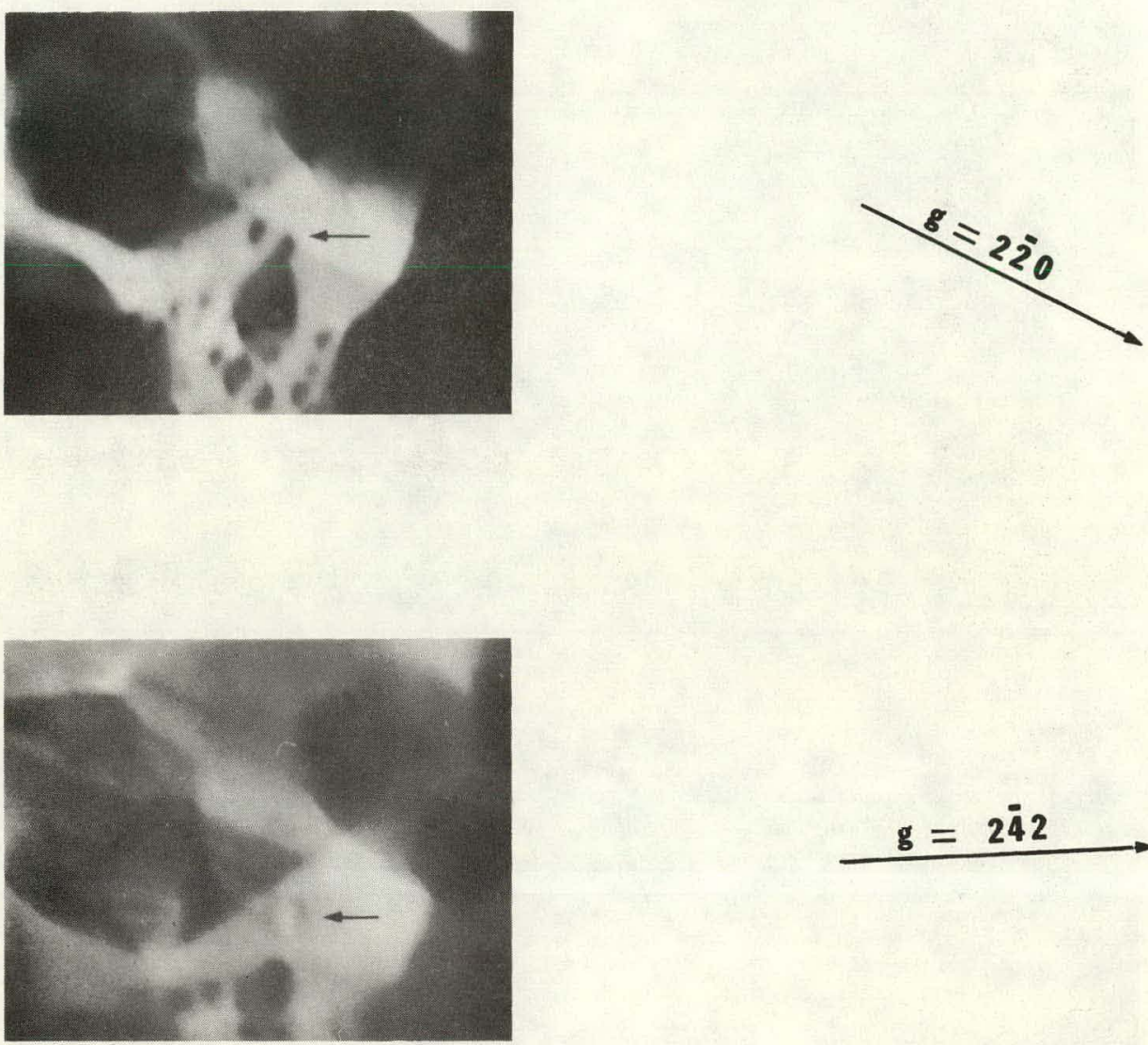

$g=2 \ddot{4} 2$
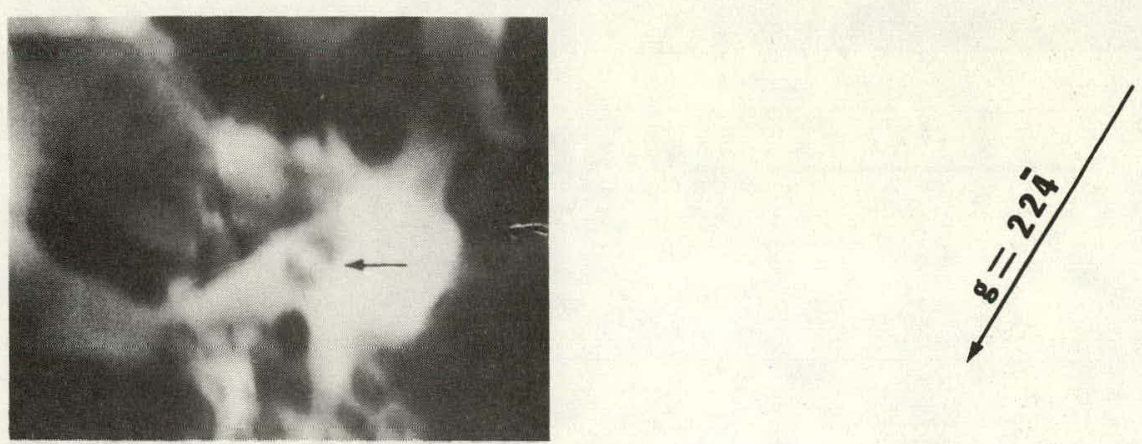

XBB709-4014

Figure 41 


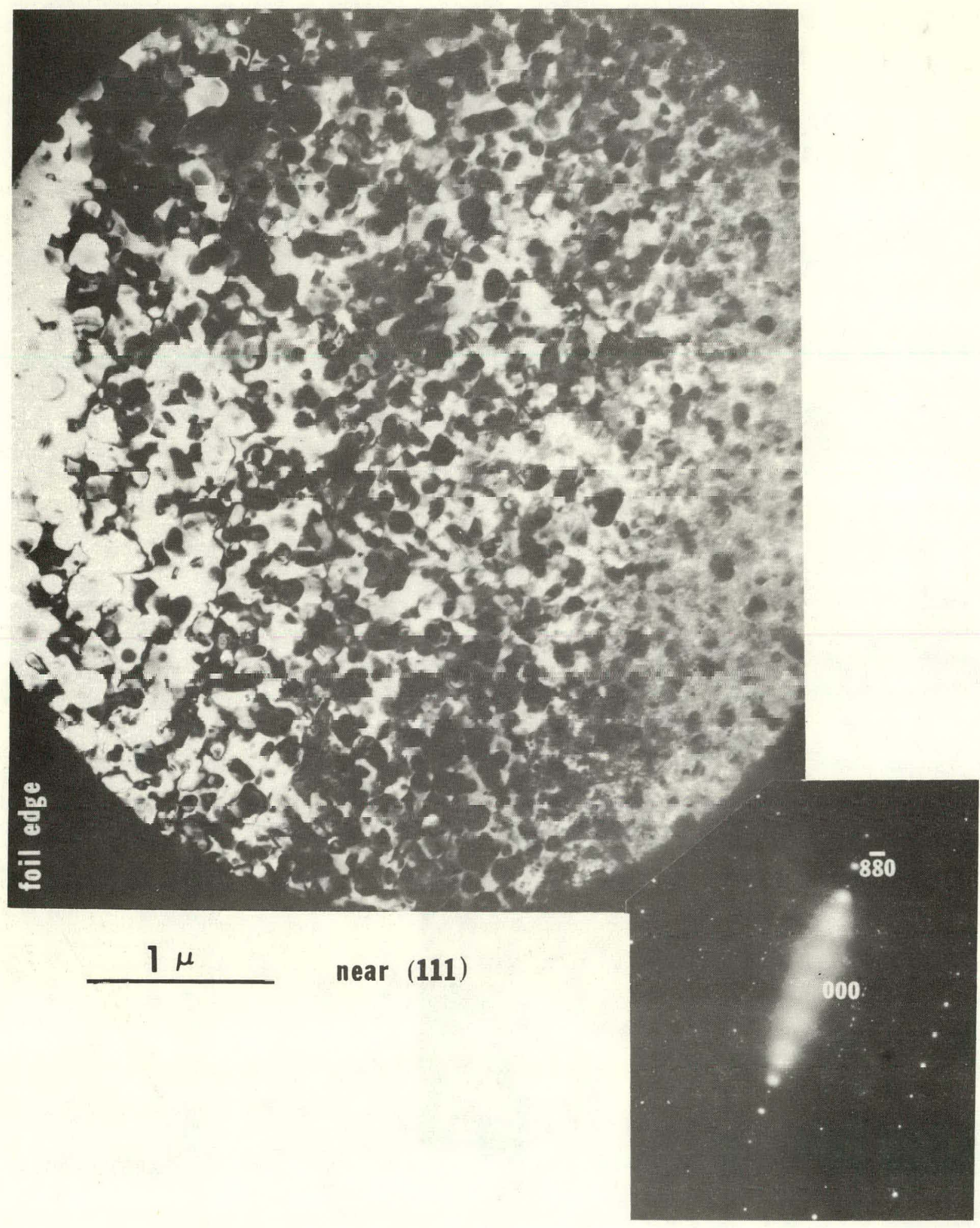

XBB709-4013

Figure 42 


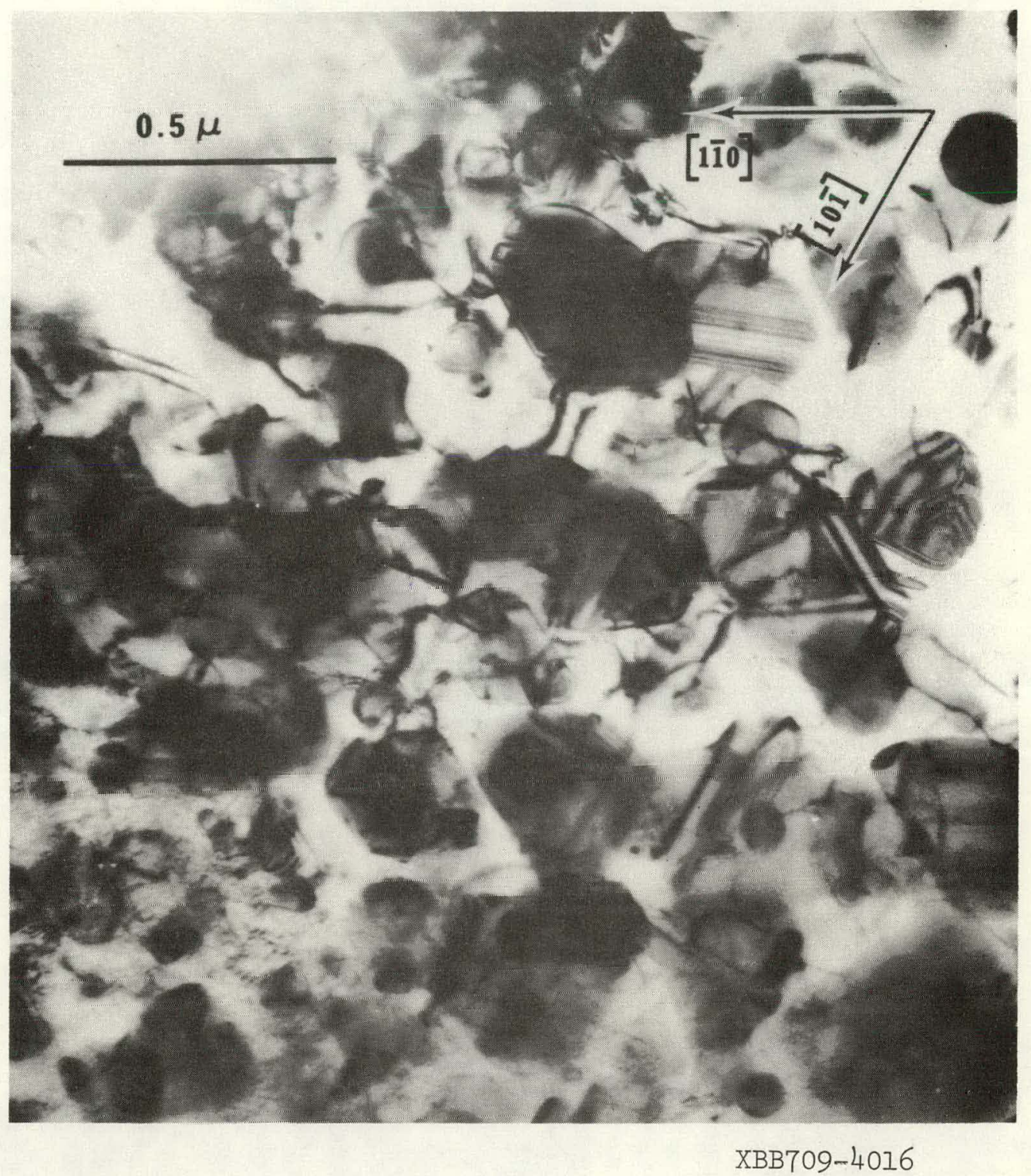

Figure 43 

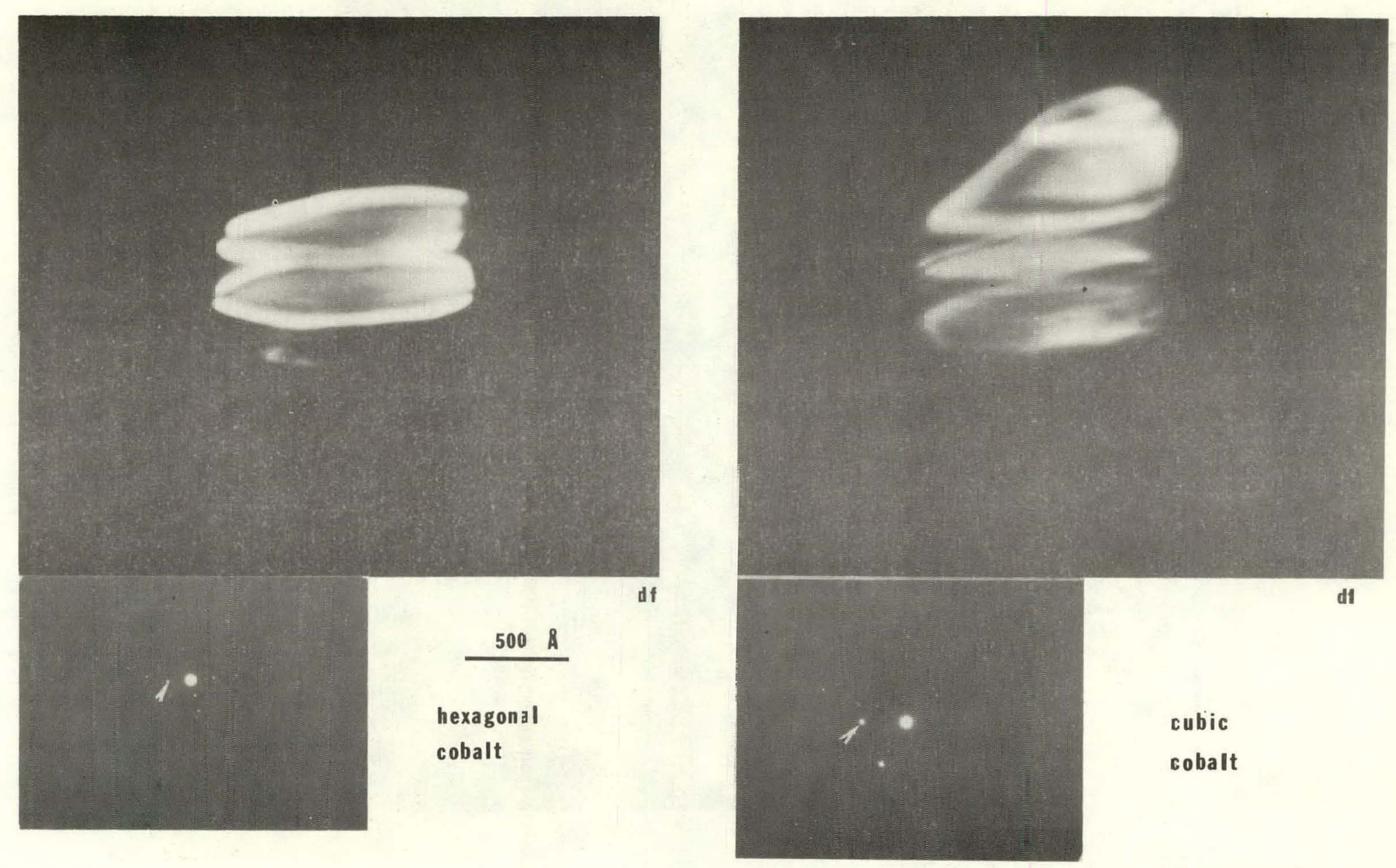

xBB709-3936

Fignre 44 

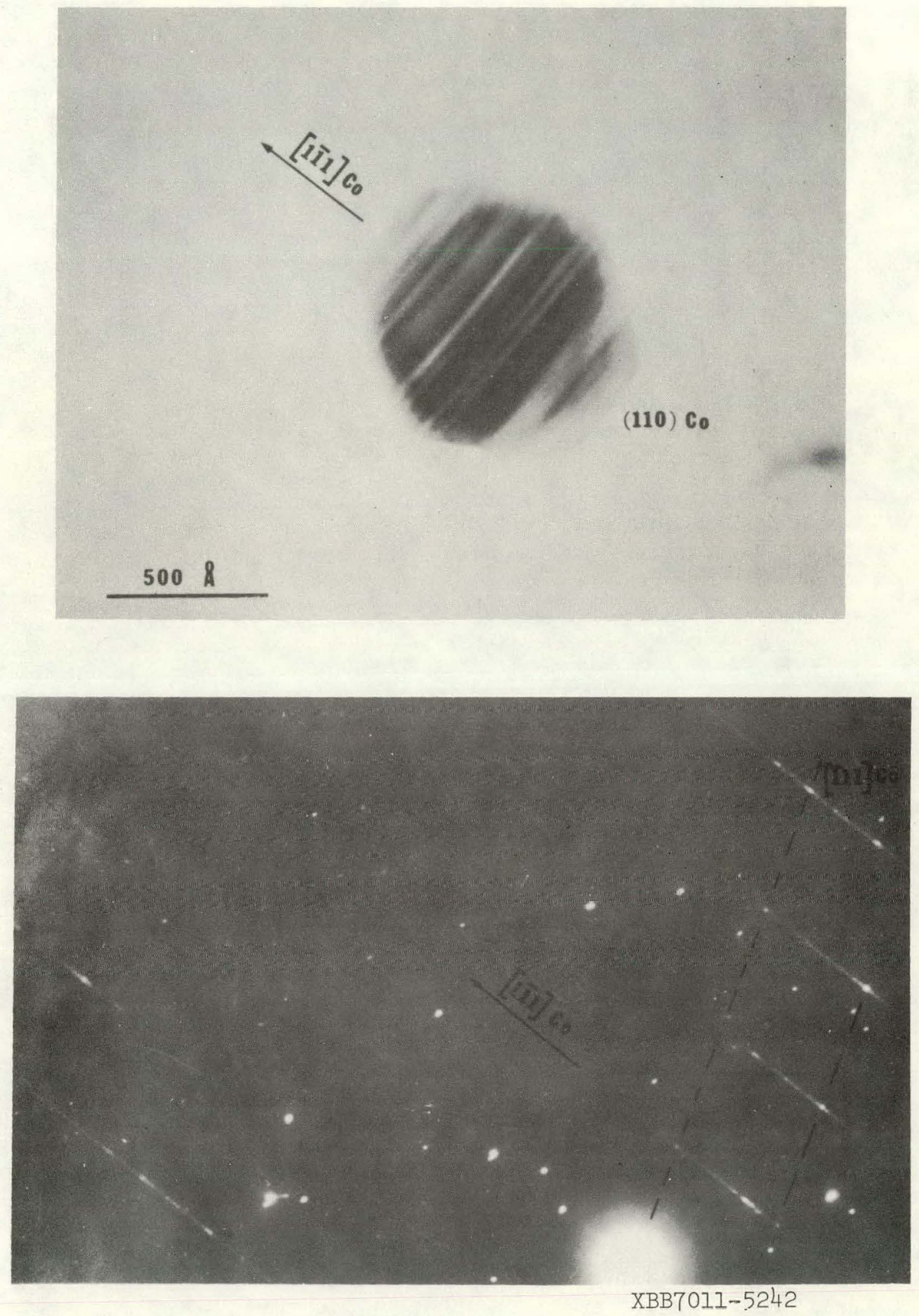

Figure 45 


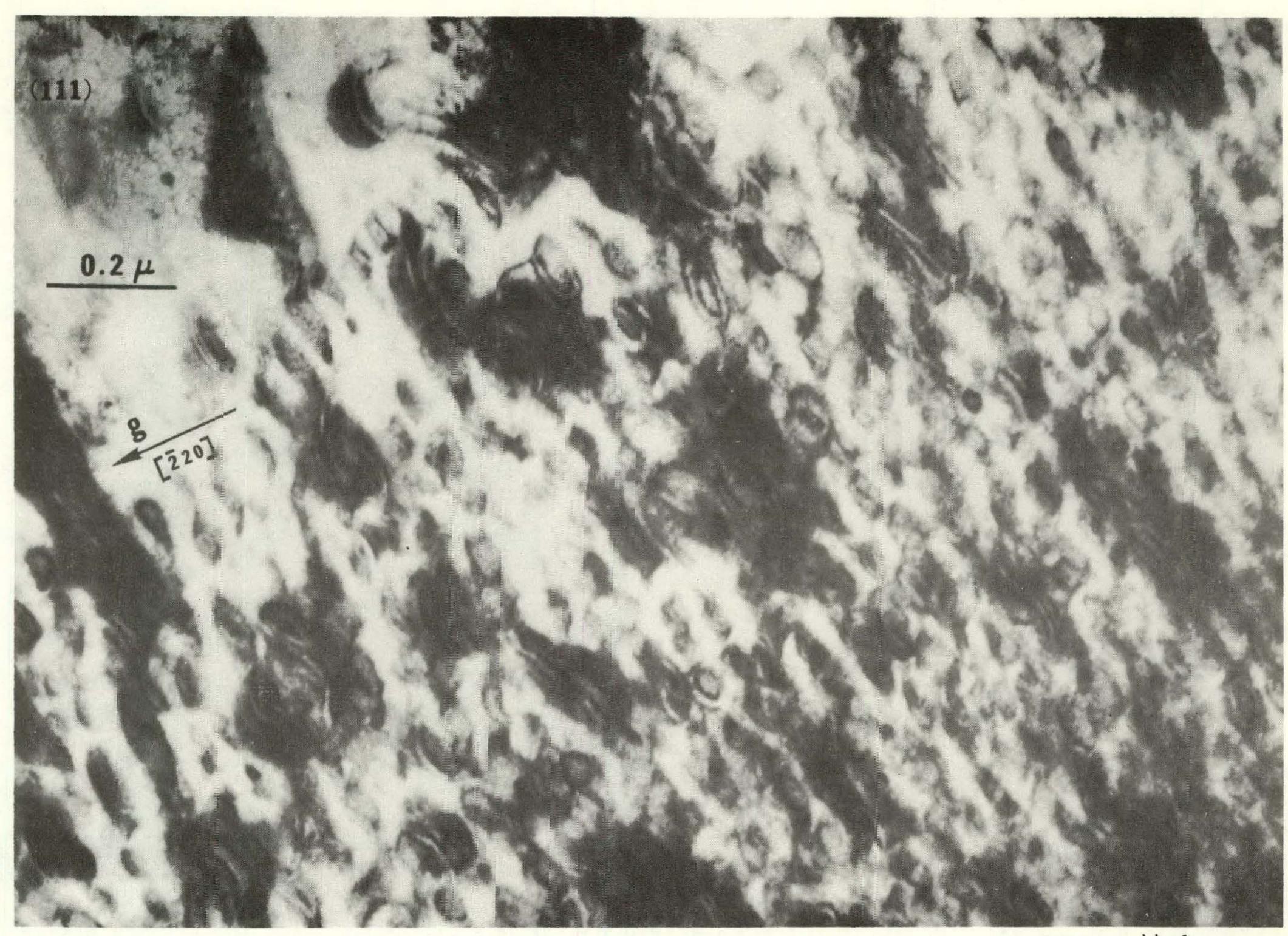

XBB7010-4498

Figure 46 
-125-
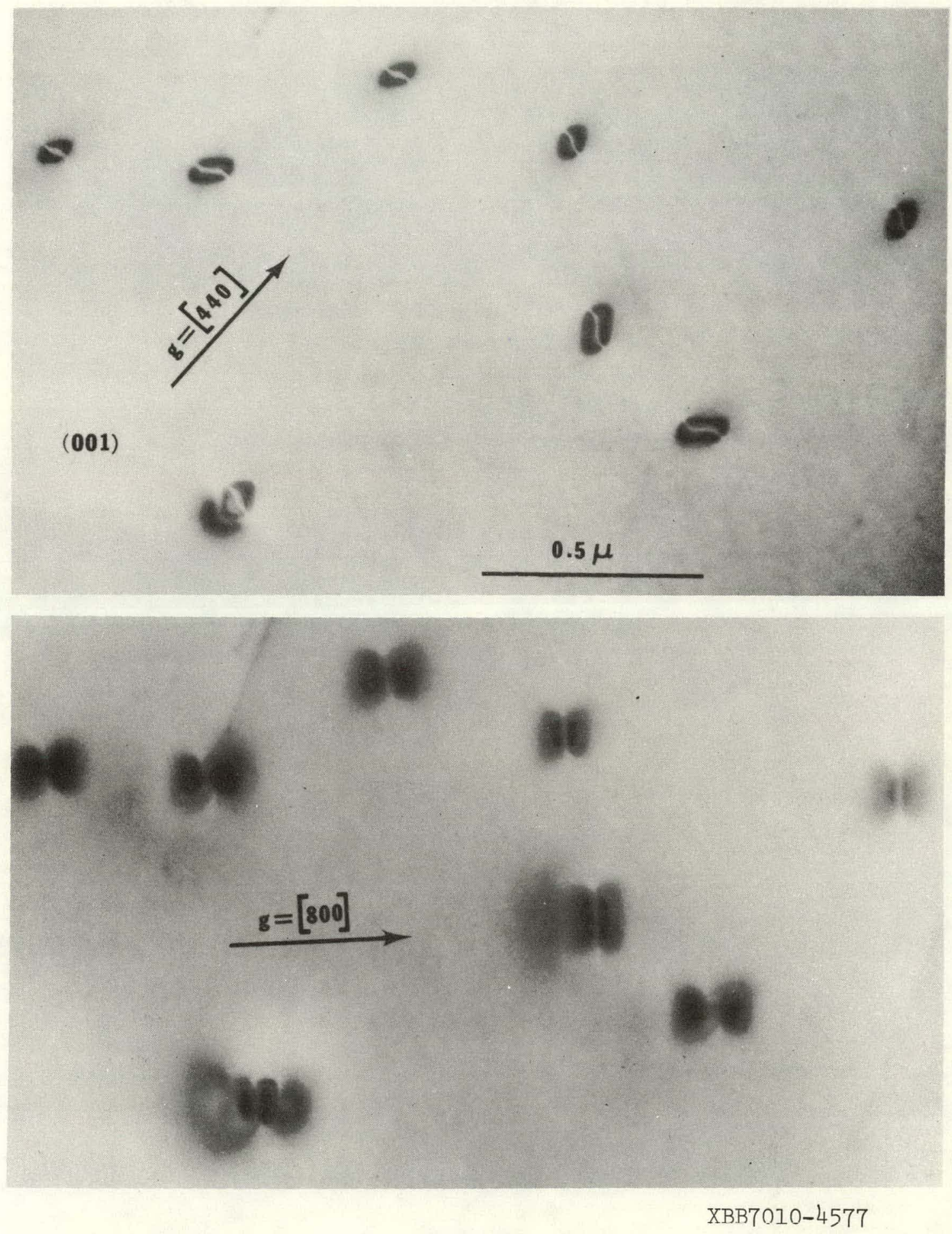

Figure 47 

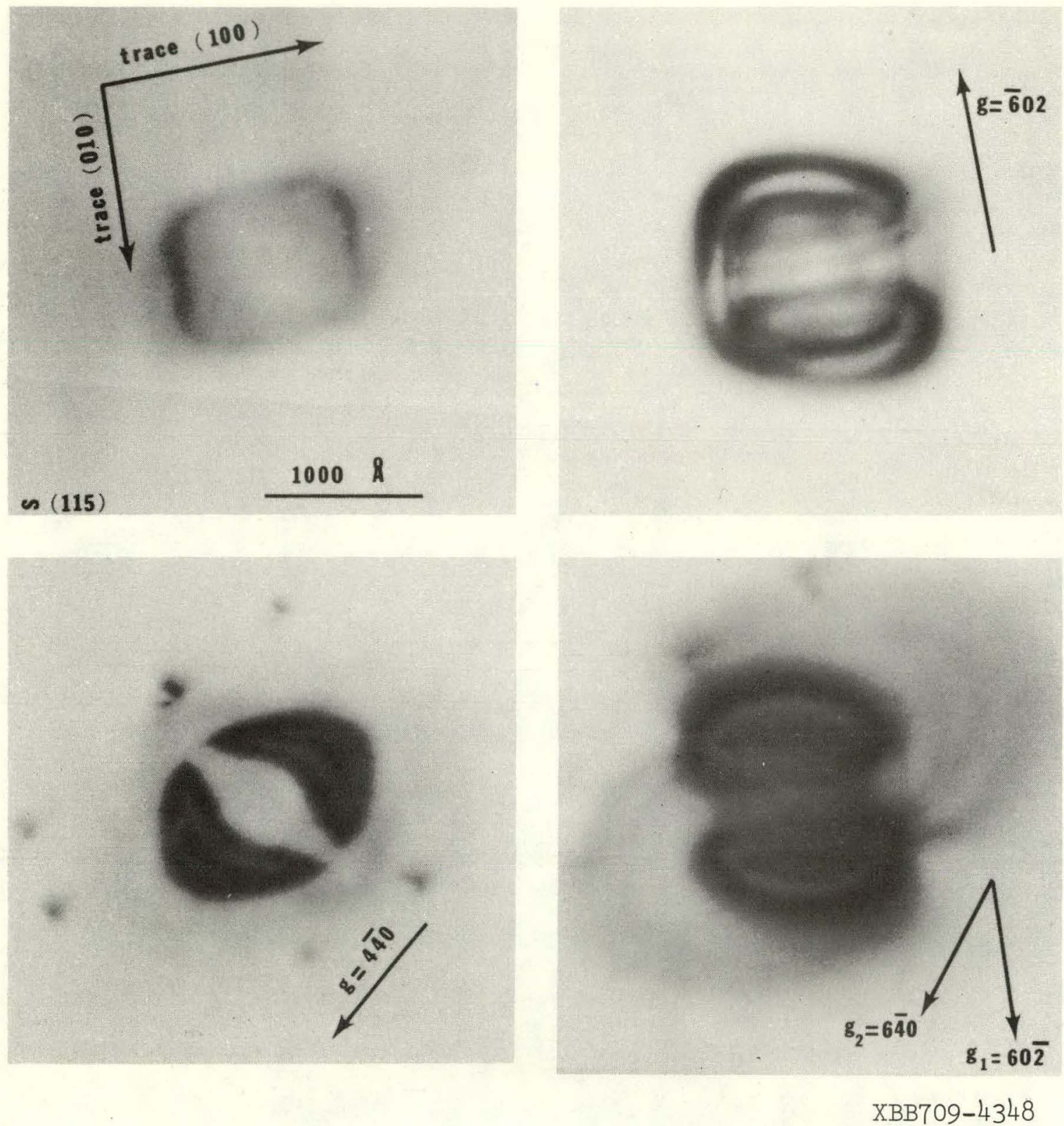

H'igure 48 




XBB7010-4578

Figure 49 
$-128-$

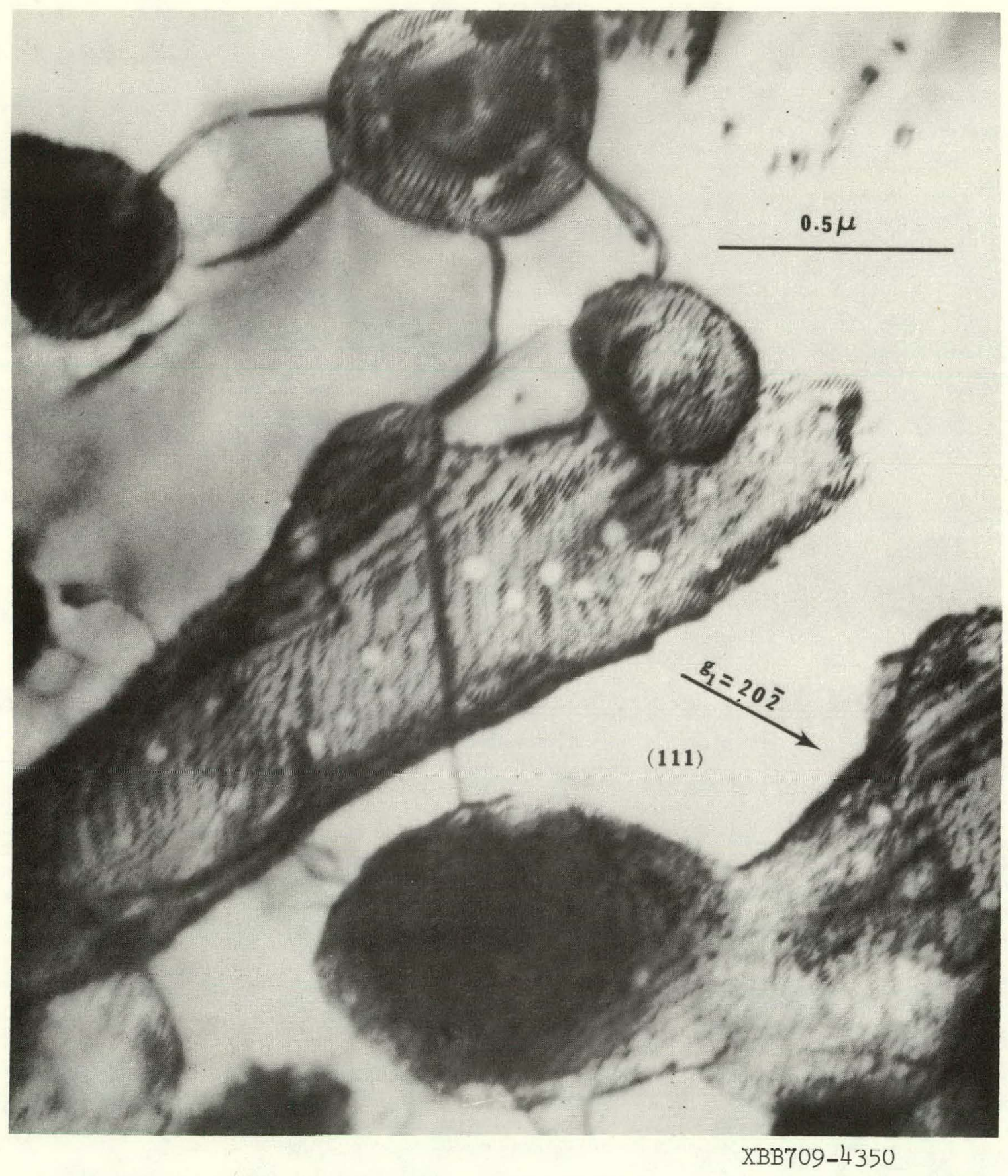

Figure 50 

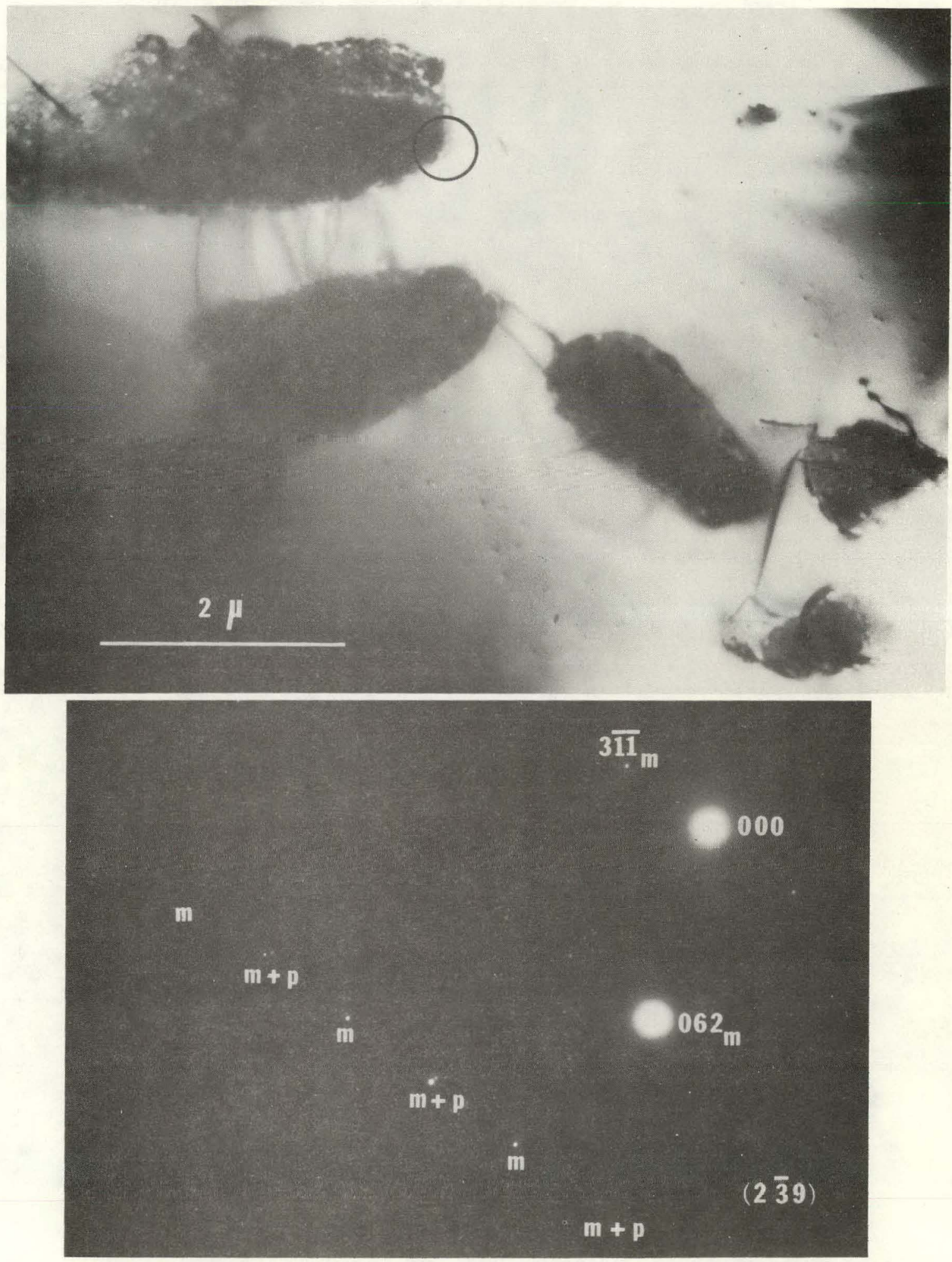

XBB709-4340 


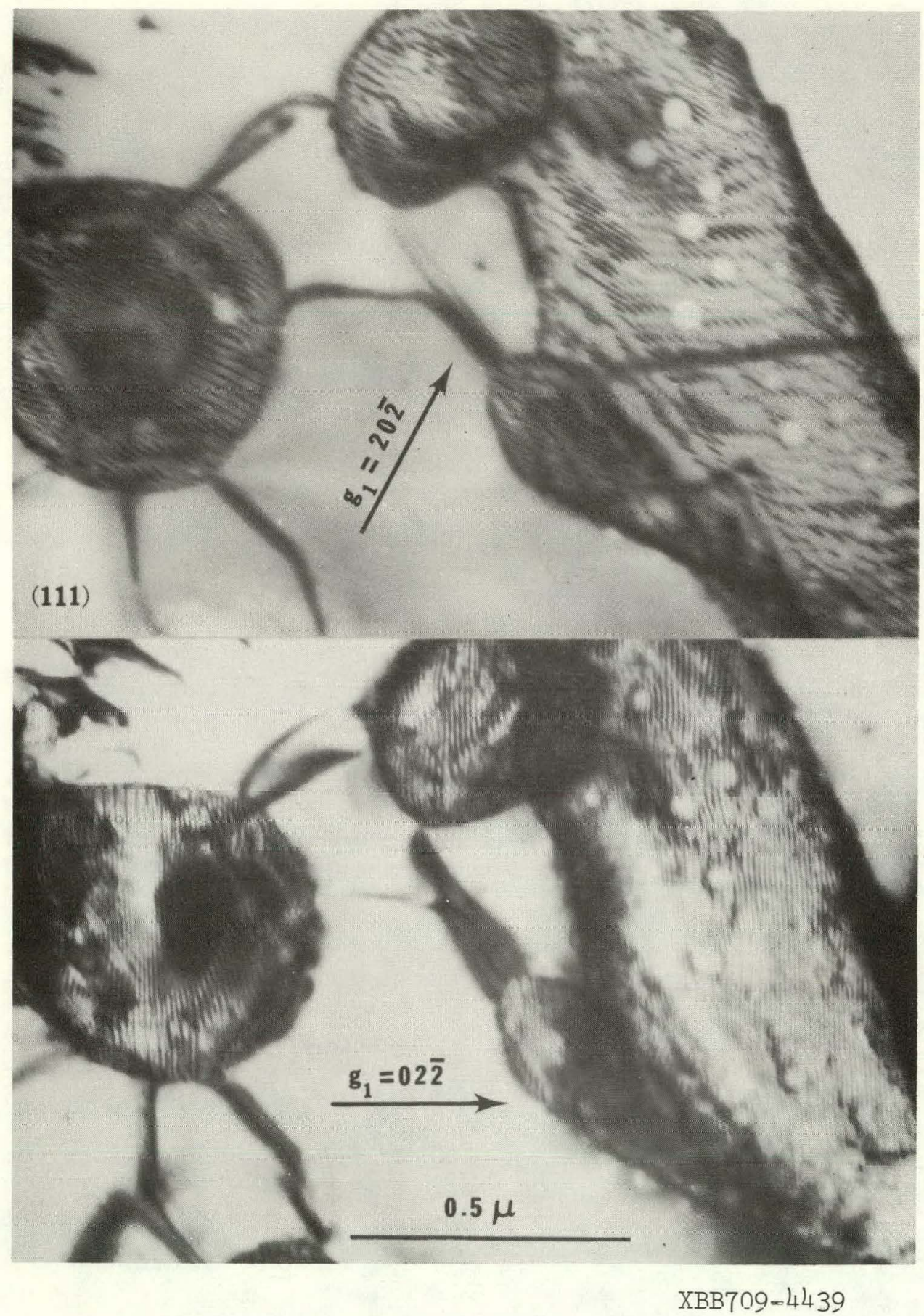

Figure 52 

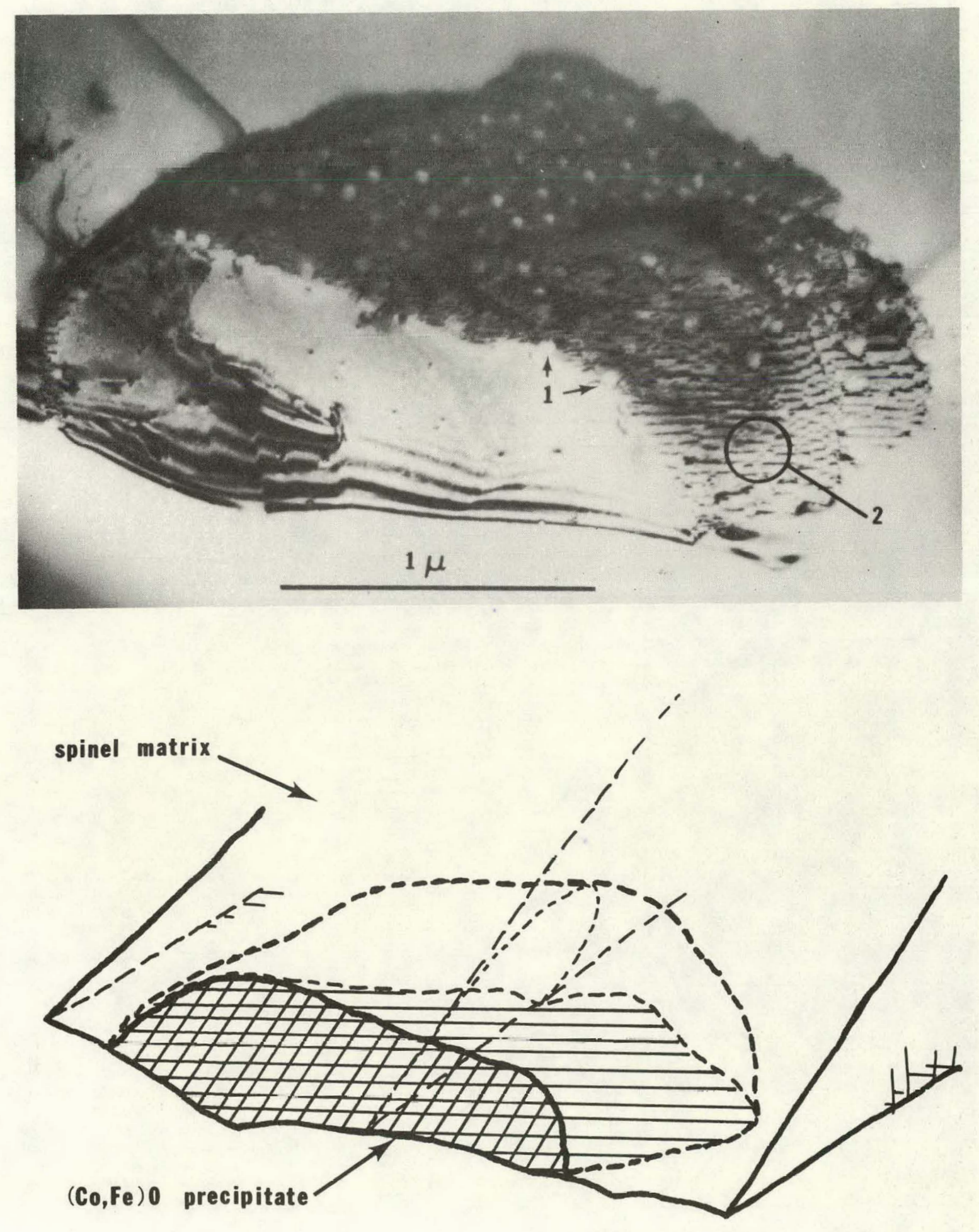

XBB709-4344 


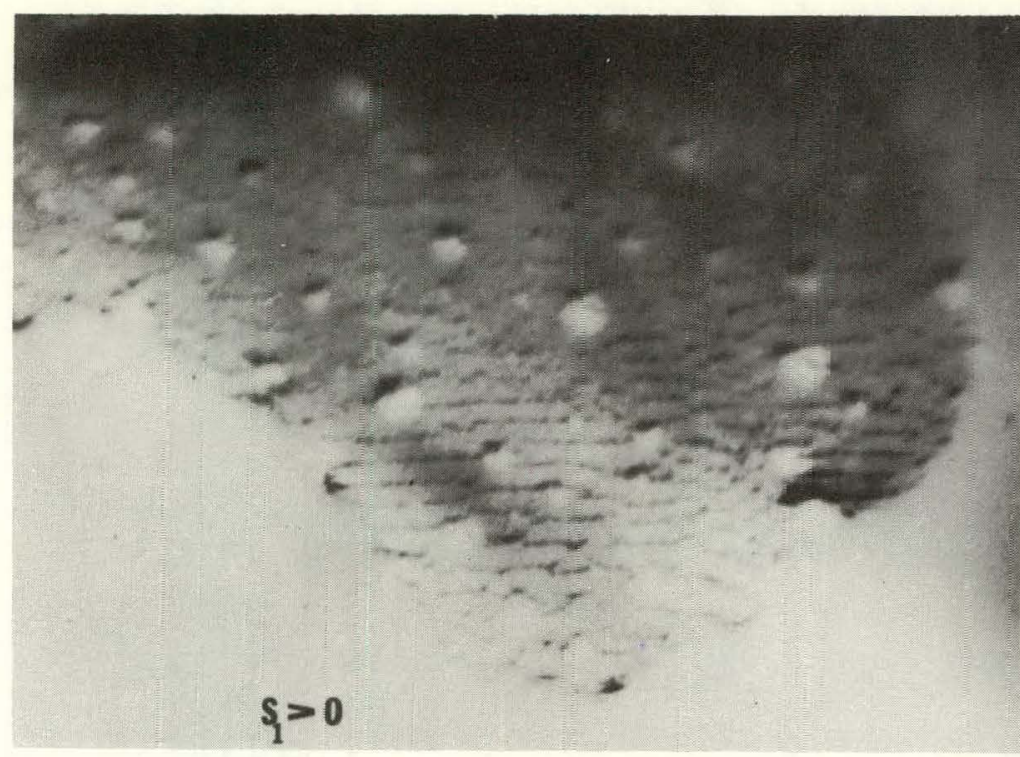

Figure 54
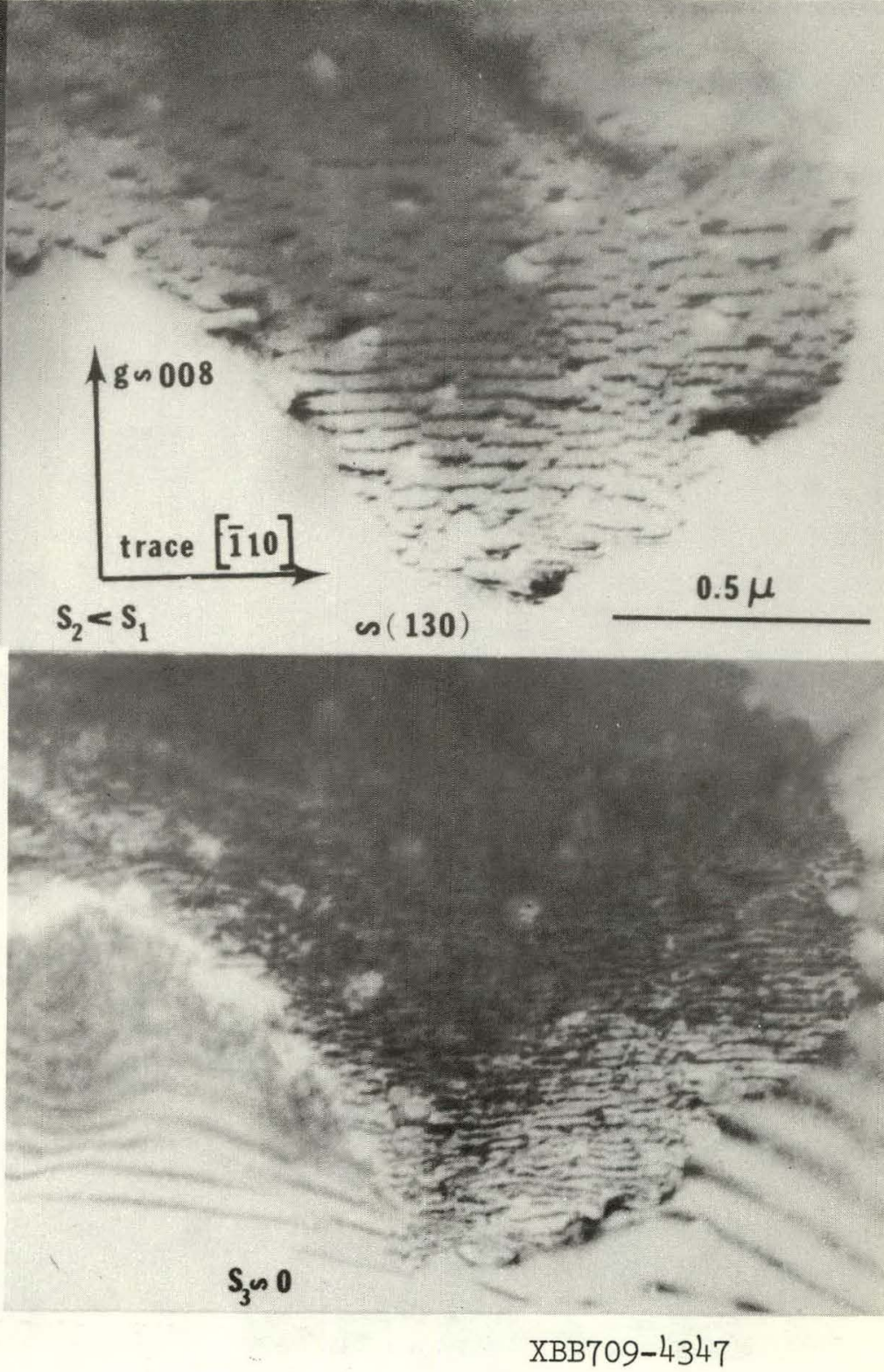

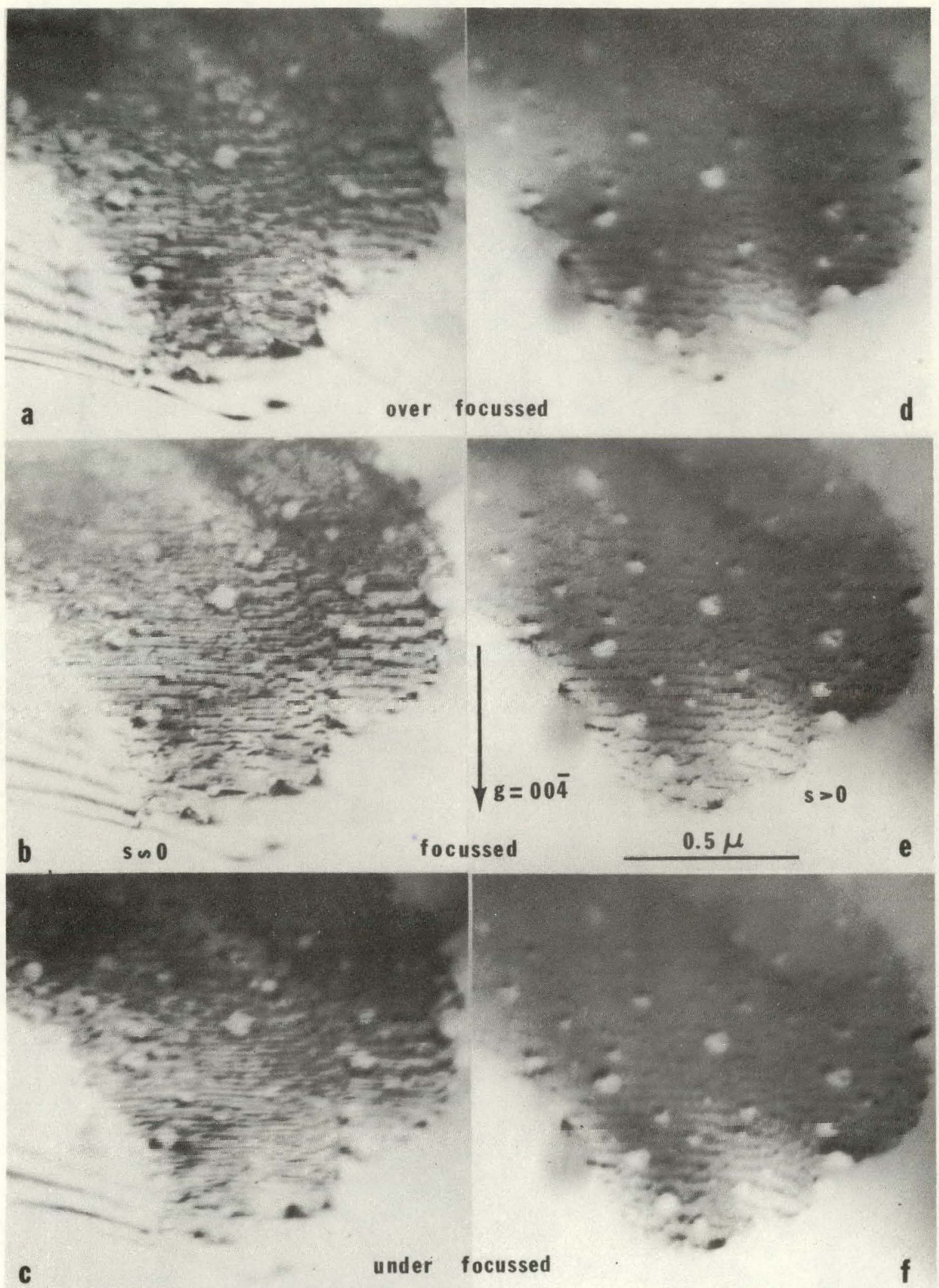

XBB709-4345

Figure 55 

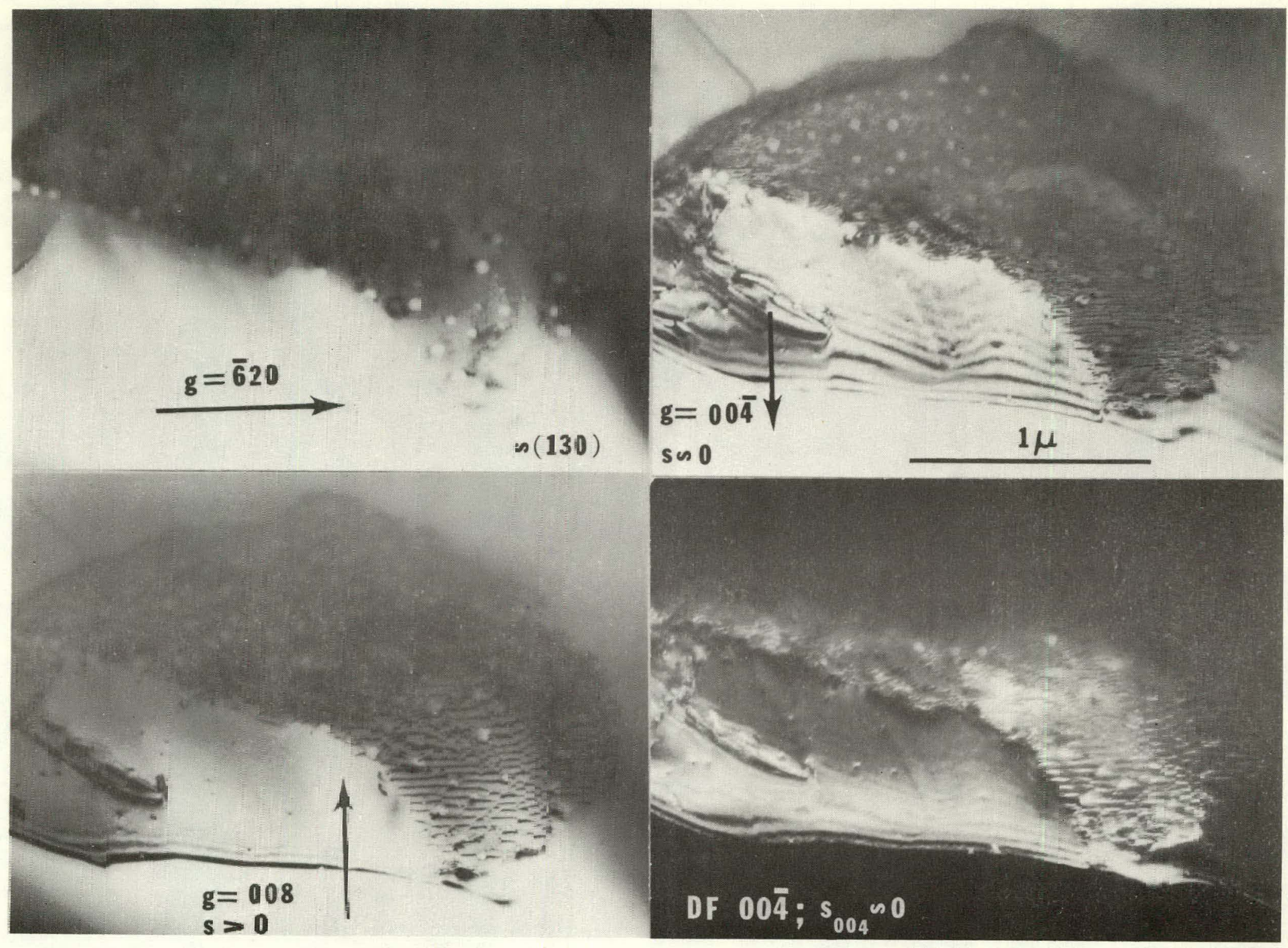


\section{LEGAL NOTICE}

This repoit was prepared as an account of Government sponsored work. Neither the United States, nor the Commission, nor any person acting on behalf of the Commission:

A. Makes any warranty or representation, expressed or implied, with respect to the accuracy, completeness, or usefulness of the information contained in this report, or that the use of any information, apparatus, method, or process disclosed in this report may not infringe privately owned rights; or

B. Assumes any liabilities with respect to the use of, or for damages resulting from the use of any information, apparatus, method, or process disclosed in this report.

As used in the above, "person acting on behalf of the Commission" includes any employee or contractor of the Commission, or employee of such contractor, to the extent that such employee or contractor of the Commission, or employee of such contractor prepares, disseminates, or provides access to, any information pursuant to his employment or contract with the Commission, or his employment with such contractor. 
TECHNICAL INFORMATION DIVISION
LAWRENCE RADIATION LABORATORY

UNIVERSITY OF CALIFORNIA
BERKELEY, CALIFORNIA 94720 